\title{
A theory of quaternionic algebra, with applications to hypercomplex geometry
}

\author{
Dominic Joyce, Lincoln College, Oxford
}

\section{Introduction}

Mathematicians are like Frenchmen: whenever you say something to them, they translate it into their own language, and at once it is something entirely different.

Goethe, Maxims and Reflections (1829)

The subject of this paper is an algebraic device, a means to construct algebraic structures over the quaternions $\mathbb{H}$ as though $\mathbb{H}$ were a commutative field. As far as the author can tell, the idea seems to be new. We shall provide the reader with a dictionary, giving the equivalents of simple concepts such as commutative field, vector space, tensor products of vector spaces, symmetric and antisymmetric products, dimensions of vector spaces, and so on. The reader will then be able to translate her own favourite algebraic objects into this new quaternionic language. The results often turn out to be surprisingly, entirely different.

The basic building blocks of the theory are the quaternionic analogues of vector space and the tensor product of vector spaces over a commutative field. A vector space is replaced by an AHI-module $\left(U, U^{\prime}\right)$, which is a left $\mathbb{H}$-module $U$ with a given real vector subspace $U^{\prime}$. The tensor product $\otimes$ is replaced by the quaternionic tensor product $\otimes_{\mathbb{H}}$, which has a complex definition given in $\S[1.1$. It shares some important properties of $\otimes$ (e.g. it is commutative and associative), but also has important differences (e.g. the dimension of a quaternionic tensor product behaves strangely).

The theory arose out of my attempts to understand the algebraic structure of noncompact hypercomplex manifolds. Let $M$ be a $4 n$-manifold. A 
hypercomplex structure on $M$ is a triple $\left(I_{1}, I_{2}, I_{3}\right)$ of complex structures satisfying $I_{1} I_{2}=I_{3}$. These induce an $\mathbb{H}$-action on the tangent bundle $T M$, and $M$ is called a hypercomplex manifold. Hypercomplex manifolds are the subject of the second half of the paper.

The best quaternionic analogue of a holomorphic function on a complex manifold is a $q$-holomorphic function on a hypercomplex manifold $M$, defined in 3.1 . This is an $\mathbb{H}$-valued function on $M$ satisfying an equation analogous to the Cauchy-Riemann equations, which was introduced by Fueter in 1935 for the case $M=\mathbb{H}$. Affine algebraic geometry is the study of complex manifolds using algebras of holomorphic functions upon them. Seeking to generalize this to hypercomplex manifolds using q-holomorphic functions, I was led to this quaternionic theory of algebra as the best language to describe quaternionic algebraic geometry.

Although the applications are all to hypercomplex manifolds, I hope that much of the paper will be of interest to those who study algebra rather than geometry, and maybe to others who, like myself, are fascinated by the quaternions. The paper has been laid out with this in mind. There are four chapters. Chapter 1 explains AHI-modules and the quaternionic tensor product. It is quite long and wholly algebraic, without references to geometry. Chapter 2 gives quaternionic analogues of various algebraic structures. It is short and is mostly definitions. The most important idea is that of an Halgebra, the quaternionic version of a commutative algebra.

Chapter 3 is about hypercomplex geometry. Q-holomorphic functions are defined, their properties explored, and it is shown that the q-holomorphic functions on a hypercomplex manifold form an $\mathrm{H}$-algebra. A similar result is proved for hyperkähler manifolds. The problem of reconstructing a hypercomplex manifold from its $\mathrm{H}$-algebra is considered, and $\mathrm{H}$-algebras are used to study a special class of noncompact, complete hyperkähler manifolds, called asymptotically conical manifolds.

Chapter 4 is a collection of examples and applications of the theory. Two interesting topics covered here are an algebraic treatment of the 'coadjoint orbit' hyperkähler manifolds, and some new types of singularities of hypercomplex manifolds that have remarkable properties. To control the length of the paper I have kept the list of examples and applications short, and I have omitted a number of proofs. However, I believe that there is much interesting work still to be done on these ideas, and in $\$ 4.7$ I shall indicate some directions in which I would like to see the subject develop.

Much of the material in this paper has already been published by the 
author in [14]; this paper is mostly an expanded version of [14], with rather more detail, and some new material on the geometry of noncompact hypercomplex and hyperkähler manifolds. However, the results of [14, §12] on coadjoint orbits are not included in this paper, and instead we approach the subject from a slightly different direction.

Two other references on the subject of this paper are Quillen [25], who reinterprets the $\mathrm{A} \mathbb{H}$-modules and the quaternionic tensor product $\otimes_{\mathbb{H}}$ in terms of sheaves on $\mathbb{C P}^{1}$, and Widdows 28 , who explores a number of issues in hypercomplex algebraic geometry, including the classification of finitedimensional AHH-modules up to isomorphism, and quaternionic analogues of the Dolbeault double complex on complex manifolds.

\subsection{Quaternionic tensor products}

In this section we give some notation, and define a quaternionic analogue of the tensor product of two vector spaces. This idea is central to the whole paper. First, a remark about the real tensor product. Let $U, V$ be real vector spaces. If $U, V$ are infinite-dimensional, there is more than one possible definition for the real tensor product $U \otimes V$, if $U, V$ are equipped with topologies. In this paper we choose the simplest definition: for us, every element of $U \otimes V$ is a finite sum $\Sigma_{j} u_{j} \otimes v_{j}$ where $u_{j} \in U, v_{j} \in V$. Also, in this paper the dual $U^{*}$ of $U$ means the vector space of all linear maps $U \rightarrow \mathbb{R}$, whether continuous in some topology or not.

The quaternions are

$$
\mathbb{H}=\left\{r_{0}+r_{1} i_{1}+r_{2} i_{2}+r_{3} i_{3}: r_{0}, \ldots, r_{3} \in \mathbb{R}\right\},
$$

and quaternion multiplication is given by

$$
i_{1} i_{2}=-i_{2} i_{1}=i_{3}, i_{2} i_{3}=-i_{3} i_{2}=i_{1}, i_{3} i_{1}=-i_{1} i_{3}=i_{2}, i_{1}^{2}=i_{2}^{2}=i_{3}^{2}=-1 .
$$

The quaternions are an associative, noncommutative algebra. The imaginary quaternions are $\mathbb{I}=\left\langle i_{1}, i_{2}, i_{3}\right\rangle$. This notation $\mathbb{I}$ is not standard, but we will use it throughout the paper. If $q=r_{0}+r_{1} i_{1}+r_{2} i_{2}+r_{3} i_{3}$ then we define the conjugate $\bar{q}$ of $q$ by $\bar{q}=r_{0}-r_{1} i_{1}-r_{2} i_{2}-r_{3} i_{3}$. Then $\overline{(p q)}=\bar{q} \bar{p}$ for $p, q \in \mathbb{H}$.

A (left) $\mathbb{H}$-module is a real vector space $U$ with an action of $\mathbb{H}$ on the left. We write this action $(q, u) \mapsto q \cdot u$ or $q u$, for $q \in \mathbb{H}$ and $u \in U$. The action is a bilinear map $\mathbb{H} \times U \rightarrow U$, and satisfies $p \cdot(q \cdot u)=(p q) \cdot u$ for $p, q \in \mathbb{H}$ and $u \in U$. In this paper, all $\mathbb{H}-$-modules will be left $\mathbb{H}$-modules. 
Let $U$ be an $\mathbb{H}$-module. We define the dual $\mathbb{H}$-module $U^{\times}$to be the vector space of linear maps $\alpha: U \rightarrow \mathbb{H}$ that satisfy $\alpha(q u)=q \alpha(u)$ for all $q \in \mathbb{H}$ and $u \in U$. If $q \in \mathbb{H}$ and $\alpha \in U^{\times}$we may define $q \cdot \alpha$ by $(q \cdot \alpha)(u)=\alpha(u) \bar{q}$ for $u \in U$. Then $q \cdot \alpha \in U^{\times}$, and $U^{\times}$is a (left) $\mathbb{H}$-module. If $V$ is a real vector space, we write $V^{*}$ for the dual of $V$ as a real vector space. It is important to distinguish between the dual vector spaces and dual $\mathbb{H}$-modules. Dual $\mathbb{H}-$ modules behave just like dual vector spaces. In particular, there is a canonical map $U \rightarrow\left(U^{\times}\right)^{\times}$, that is an isomorphism when $U$ is finite-dimensional.

Definition 1.1.1 Let $U$ be an $\mathbb{H}$-module. Let $U^{\prime}$ be a real vector subspace of $U$, that need not be closed under the $\mathbb{H}$-action. Define a real vector subspace $U^{\dagger}$ of $U^{\times}$by

$$
U^{\dagger}=\left\{\alpha \in U^{\times}: \alpha(u) \in \mathbb{I} \text { for all } u \in U^{\prime}\right\} .
$$

We define an augmented $\mathbb{H}-$-module, or A $\mathbb{H}$-module, to be a pair $\left(U, U^{\prime}\right)$, such that if $u \in U$ and $\alpha(u)=0$ for all $\alpha \in U^{\dagger}$, then $u=0$. Usually we will refer to $U$ as an $\mathrm{AH}$-module, implicitly assuming that $U^{\prime}$ is also given. We consider $\mathbb{H}$ to be an $A \mathbb{H}$-module, with $\mathbb{H}^{\prime}=\mathbb{I}$.

$\mathrm{AH}$-modules should be thought of as the quaternionic analogues of real vector spaces. It is easy to define the dual of an A $\mathbb{H}$-module, but we are not going to do this, as it seems not to be a fruitful idea. We can interpret $U^{\times}$ as the dual of $U$ as a real vector space, and then $U^{\dagger}$ is the annihilator of $U^{\prime}$. Thus if $U$ is finite-dimensional, $\operatorname{dim} U^{\prime}+\operatorname{dim} U^{\dagger}=\operatorname{dim} U=\operatorname{dim} U^{\times}$. The letters $A, U, V, \ldots, Z$ will usually denote $\mathrm{A} \mathbb{H}$-modules. Here are the natural concepts of linear map between AHI-modules, and AHI-submodules.

Definition 1.1.2 Let $U, V$ be AHH-modules. Let $\phi: U \rightarrow V$ be a linear map satisfying $\phi(q u)=q \phi(u)$ for each $q \in \mathbb{H}$ and $u \in U$. Such a map is called quaternion linear, or $\mathbb{H}$-linear. We say that $\phi$ is a morphism of AHI-

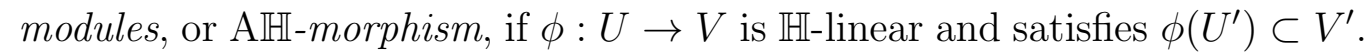
Define a linear map $\phi^{\times}: V^{\times} \rightarrow U^{\times}$by $\phi^{\times}(\beta)(u)=\beta(\phi(u))$ for $\beta \in V^{\times}$and $u \in U$. Then $\phi\left(U^{\prime}\right) \subset V^{\prime}$ implies that $\phi^{\times}\left(V^{\dagger}\right) \subset U^{\dagger}$. If $\phi$ is an isomorphism of $\mathbb{H}$-modules and $\phi\left(U^{\prime}\right)=V^{\prime}$, we say that $\phi$ is an $\mathrm{A} \mathbb{H}$-isomorphism.

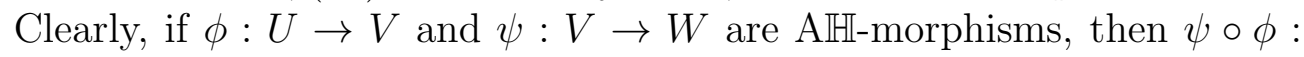

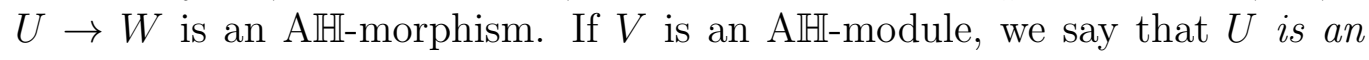
AHH-submodule of $V$ if $U$ is an $\mathbb{H}$-submodule of $V$ and $U^{\prime}=U \cap V^{\prime}$. This implies that $U^{\dagger}$ is the restriction of $V^{\dagger}$ to $U$, so the condition that $u=0$ if $\alpha(u)=0$ for all $\alpha \in U^{\dagger}$ holds automatically, and $U$ is an AHI-module. 
In this paper 'Im' always means the image of a map, and we will write 'id' for the identity map on any vector space. If $U$ is an AHI-module, then id : $U \rightarrow U$ is an A $\mathbb{H}$-morphism. Next we will define a sort of tensor product of $\mathrm{A} \mathbb{H}$-modules, which is the key to the whole paper.

Definition 1.1.3 Let $U$ be an AHH-module. Then $\mathbb{H} \otimes\left(U^{\dagger}\right)^{*}$ is an $\mathbb{H}$-module, with $\mathbb{H}$-action $p \cdot(q \otimes x)=(p q) \otimes x$. Define a map $\iota_{U}: U \rightarrow \mathbb{H} \otimes\left(U^{\dagger}\right)^{*}$ by $\iota_{U}(u) \cdot \alpha=\alpha(u)$, for $u \in U$ and $\alpha \in U^{\dagger}$. Then $\iota_{U}(q \cdot u)=q \cdot \iota_{U}(u)$ for $u \in U$. Thus $\iota_{U}(U)$ is an $\mathbb{H}$-submodule of $\mathbb{H} \otimes\left(U^{\dagger}\right)^{*}$. Suppose $u \in \operatorname{Ker} \iota_{U}$. Then $\alpha(u)=0$ for all $\alpha \in U^{\dagger}$, so that $u=0$ as $U$ is an AHH-module. Thus $\iota_{U}$ is injective, and $\iota_{U}(U) \cong U$.

Definition 1.1.4 Let $U, V$ be AHH-modules. Then $\mathbb{H} \otimes\left(U^{\dagger}\right)^{*} \otimes\left(V^{\dagger}\right)^{*}$ is an $\mathbb{H}$-module, with $\mathbb{H}$-action $p \cdot(q \otimes x \otimes y)=(p q) \otimes x \otimes y$. Exchanging the factors of $\mathbb{H}$ and $\left(U^{\dagger}\right)^{*}$, we may regard $\left(U^{\dagger}\right)^{*} \otimes \iota_{V}(V)$ as a subspace of $\mathbb{H} \otimes\left(U^{\dagger}\right)^{*} \otimes\left(V^{\dagger}\right)^{*}$. Thus $\iota_{U}(U) \otimes\left(V^{\dagger}\right)^{*}$ and $\left(U^{\dagger}\right)^{*} \otimes \iota_{V}(V)$ are $\mathbb{H}$-submodules of $\mathbb{H} \otimes\left(U^{\dagger}\right)^{*} \otimes\left(V^{\dagger}\right)^{*}$. Define an $\mathbb{H}$-module $U \otimes_{\mathbb{H}} V$ by

$$
U \otimes_{\mathbb{H}} V=\left(\iota_{U}(U) \otimes\left(V^{\dagger}\right)^{*}\right) \cap\left(\left(U^{\dagger}\right)^{*} \otimes \iota_{V}(V)\right) \subset \mathbb{H} \otimes\left(U^{\dagger}\right)^{*} \otimes\left(V^{\dagger}\right)^{*} .
$$

Define a vector subspace $\left(U \otimes_{\mathbb{H}} V\right)^{\prime}$ by $\left(U \otimes_{\mathbb{H}} V\right)^{\prime}=\left(U \otimes_{\mathbb{H}} V\right) \cap\left(\mathbb{I} \otimes\left(U^{\dagger}\right)^{*} \otimes\right.$ $\left.\left(V^{\dagger}\right)^{*}\right)$. Define a linear map $\lambda_{U, V}: U^{\dagger} \otimes V^{\dagger} \rightarrow\left(U \otimes_{\mathbb{H}} V\right)^{\times}$by $\lambda_{U, V}(x)(y)=$ $y \cdot x \in \mathbb{H}$, for $x \in U^{\dagger} \otimes V^{\dagger}, y \in U \otimes_{\mathbb{H}} V$, where '.' contracts together the factors of $U^{\dagger} \otimes V^{\dagger}$ and $\left(U^{\dagger}\right)^{*} \otimes\left(V^{\dagger}\right)^{*}$.

Clearly, if $x \in U^{\dagger} \otimes V^{\dagger}$ and $y \in\left(U \otimes_{\mathbb{H}} V\right)^{\prime}$, then $\lambda_{U, V}(x)(y) \in \mathbb{I}$. As this holds for all $y \in\left(U \otimes_{\mathbb{H}} V\right)^{\prime}, \lambda_{U, V}(x) \in\left(U \otimes_{\mathbb{H}} V\right)^{\dagger}$, so that $\lambda_{U, V}$ maps $U^{\dagger} \otimes V^{\dagger} \rightarrow\left(U \otimes_{\mathbb{H}} V\right)^{\dagger}$. If $y \in U \otimes_{\mathbb{H}} V$, then $\lambda_{U, V}(x)(y)=0$ for all $x \in U^{\dagger} \otimes V^{\dagger}$ if and only if $y=0$. Thus $U \otimes_{\mathbb{H}} V$ is an AH-module, by Definition 1.1.1. This A $\mathbb{H}$-module will be called the quaternionic tensor product of $U$ and $V$, and the operation $\otimes_{\mathbb{H}}$ will be called the quaternionic tensor product. When $U, V$ are finite-dimensional, $\lambda_{U, V}$ is surjective, so that $\left(U \otimes_{\mathbb{H}} V\right)^{\dagger}=\lambda_{U, V}\left(U^{\dagger} \otimes V^{\dagger}\right)$.

Here are some basic properties of the operation $\otimes_{\mathbb{H}}$.

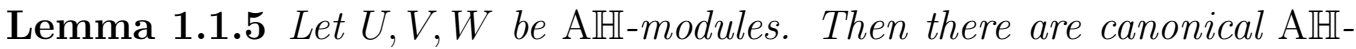
isomorphisms

$$
\mathbb{H} \otimes_{\mathbb{H}} U \cong U, \quad U \otimes_{\mathbb{H}} V \cong V \otimes_{\mathbb{H}} U, \quad \text { and } \quad\left(U \otimes_{\mathbb{H}} V\right) \otimes_{\mathbb{H}} W \cong U \otimes_{\mathbb{H}}\left(V \otimes_{\mathbb{H}} W\right) .
$$


Proof. As $\mathbb{H}^{\dagger} \cong \mathbb{R}$, we may identify $\mathbb{H} \otimes\left(\mathbb{H}^{\dagger}\right)^{*} \otimes\left(U^{\dagger}\right)^{*}$ and $\mathbb{H} \otimes\left(U^{\dagger}\right)^{*}$. Under this identification, it is easy to see that $\mathbb{H} \otimes_{\mathbb{H}} U$ and $\iota_{U}(U)$ are identified. Since $\iota_{U}(U) \cong U$ as in Definition 1.1.3, this gives an isomorphism $\mathbb{H} \otimes_{\mathbb{H}} U \cong U$,

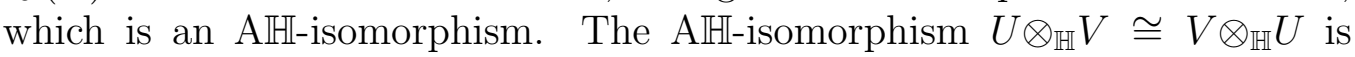
trivial, because the definition of $U \otimes_{\mathbb{H}} V$ is symmetric in $U$ and $V$.

It remains to show that $\left(U \otimes_{\mathbb{H}} V\right) \otimes_{\mathbb{H}} W \cong U \otimes_{\mathbb{H}}\left(V \otimes_{\mathbb{H}} W\right)$. The maps $\lambda_{U, V}$ : $U^{\dagger} \otimes V^{\dagger} \rightarrow\left(U \otimes_{\mathbb{H}} V\right)^{\dagger}$ and $\lambda_{U \otimes_{\mathbb{H}} V, W}:\left(U \otimes_{\mathbb{H}} V\right)^{\dagger} \otimes W^{\dagger} \rightarrow\left(\left(U \otimes_{\mathbb{H}} V\right) \otimes_{\mathbb{H}} W\right)^{\dagger}$ compose to give a linear map $\lambda_{U V, W}: U^{\dagger} \otimes V^{\dagger} \otimes W^{\dagger} \rightarrow\left(\left(U \otimes_{\mathbb{H}} V\right) \otimes_{\mathbb{H}} W\right)^{\dagger}$, defined in the obvious way. Define a linear map $\iota_{U V, W}:\left(U \otimes_{\mathbb{H}} V\right) \otimes_{\mathbb{H}} W \rightarrow$ $\mathbb{H} \otimes\left(U^{\dagger}\right)^{*} \otimes\left(V^{\dagger}\right)^{*} \otimes\left(W^{\dagger}\right)^{*}$ by $\iota_{U V, W}(y) \cdot x=\lambda_{U V, W}(x)(y) \in \mathbb{H}$, for each $x \in$ $U^{\dagger} \otimes V^{\dagger} \otimes W^{\dagger}$.

Suppose that $y \in\left(U \otimes_{\mathbb{H}} V\right) \otimes_{\mathbb{H}} W$, and $\iota_{U V, W}(y)=0$. Then $\lambda_{U V, W}(x)(y)=$ 0 for each $x \in U^{\dagger} \otimes V^{\dagger} \otimes W^{\dagger}$. It can be shown that this implies that $y=0$. Thus $\iota_{U V, W}$ is injective. Now from (11) and the definitions, it is easy to show that

$$
\begin{aligned}
\iota_{U V, W}( & \left.\left(U \otimes_{\mathbb{H}} V\right) \otimes_{\mathbb{H}} W\right)=\left(\iota_{U}(U) \otimes\left(V^{\dagger}\right)^{*} \otimes\left(W^{\dagger}\right)^{*}\right) \\
& \cap\left(\left(U^{\dagger}\right)^{*} \otimes \iota_{V}(V) \otimes\left(W^{\dagger}\right)^{*}\right) \cap\left(\left(U^{\dagger}\right)^{*} \otimes\left(V^{\dagger}\right)^{*} \otimes \iota_{W}(W)\right),
\end{aligned}
$$

interpreting this equation as we did (1). As $\iota_{U V, W}$ is injective, $\left(U \otimes_{\mathbb{H}} V\right) \otimes_{\mathbb{H}} W$ is isomorphic to the r.h.s. of (2) . By the same argument, $U \otimes_{\mathbb{H}}\left(V \otimes_{\mathbb{H}} W\right)$ is also isomorphic to the r.h.s. of (2). This gives a canonical isomorphism $\left(U \otimes_{\mathbb{H}} V\right) \otimes_{\mathbb{H}} W \cong U \otimes_{\mathbb{H}}\left(V \otimes_{\mathbb{H}} W\right)$. It turns out to be an AHI-isomorphism, and the lemma is complete.

Lemma 1.1.5 tells us that $\otimes_{\mathbb{H}}$ is commutative and associative, and that $\mathbb{H}$ acts as an identity element for $\otimes_{\mathbb{H}}$. Since $\otimes_{\mathbb{H}}$ is associative, we shall not bother to put brackets in multiple products such as $U \otimes_{\mathbb{H}} V \otimes_{\mathbb{H}} W$. Also, the commutativity and associativity of $\otimes_{\mathbb{H}}$ enable us to define symmetric and antisymmetric products, analogous to $S^{k} V$ and $\Lambda^{k} V$ for $V$ a real vector space.

Definition 1.1.6 Let $U$ be an AHI-module. Write $\bigotimes_{\mathbb{H}}^{k} U$ for the product $U \otimes_{\mathbb{H}} \cdots \otimes_{\mathbb{H}} U$ of $k$ copies of $U$. Then the $k^{\text {th }}$ symmetric group $S_{k}$ acts on $\bigotimes_{\mathbb{H}}^{k} U$ by permutation of the $U$ factors in the obvious way. Define $S_{\mathbb{H}}^{k} U$ to be the AHI-submodule of $\bigotimes_{\mathbb{H}}^{k} U$ that is symmetric under these permutations, and $\Lambda_{\mathbb{H}}^{k} U$ to be the AHI-submodule of $\otimes_{\mathbb{H}}^{k} U$ that is antisymmetric under these permutations. Define $\bigotimes_{\mathbb{H}}^{0} U, S_{\mathbb{H}}^{0} U$ and $\Lambda_{\mathbb{H}}^{0} U$ to be the AHI-module $\mathbb{H}$. The symmetrization operator $\sigma_{\mathbb{H}}$, defined in the obvious way, is a projection 
$\sigma_{\mathbb{H}}: \bigotimes_{\mathbb{H}}^{k} U \rightarrow S_{\mathbb{H}}^{k} U$. Clearly, $\sigma_{\mathbb{H}}$ is an AH-morphism. Similarly, there is an antisymmetrization operator, that is an AH-morphism projection from $\bigotimes_{\mathbb{H}}^{k} U$ to $\Lambda_{\mathbb{H}}^{k} U$.

Here is the definition of the tensor product of two AHI-morphisms.

Definition 1.1.7 Let $U, V, W, X$ be AHI-modules, and let $\phi: U \rightarrow W$ and $\psi: V \rightarrow X$ be AHI-morphisms. Then $\phi^{\times}\left(W^{\dagger}\right) \subset U^{\dagger}$ and $\psi^{\times}\left(X^{\dagger}\right) \subset V^{\dagger}$, by definition. Taking the duals gives maps $\left(\phi^{\times}\right)^{*}:\left(U^{\dagger}\right)^{*} \rightarrow\left(W^{\dagger}\right)^{*}$ and $\left(\psi^{\times}\right)^{*}:\left(V^{\dagger}\right)^{*} \rightarrow\left(X^{\dagger}\right)^{*}$. Combining these, we have a map

$$
\mathrm{id} \otimes\left(\phi^{\times}\right)^{*} \otimes\left(\psi^{\times}\right)^{*}: \mathbb{H} \otimes\left(U^{\dagger}\right)^{*} \otimes\left(V^{\dagger}\right)^{*} \rightarrow \mathbb{H} \otimes\left(W^{\dagger}\right)^{*} \otimes\left(X^{\dagger}\right)^{*} .
$$

Now $U \otimes_{\mathbb{H}} V \subset \mathbb{H} \otimes\left(U^{\dagger}\right)^{*} \otimes\left(V^{\dagger}\right)^{*}$ and $W \otimes_{\mathbb{H}} X \subset \mathbb{H} \otimes\left(W^{\dagger}\right)^{*} \otimes\left(X^{\dagger}\right)^{*}$. It is easy to show that $\left(\mathrm{id} \otimes\left(\phi^{\times}\right)^{*} \otimes\left(\psi^{\times}\right)^{*}\right)\left(U \otimes_{\mathbb{H}} V\right) \subset W \otimes_{\mathbb{H}} X$. Define $\phi \otimes_{\mathbb{H}} \psi: U \otimes_{\mathbb{H}} V \rightarrow W \otimes_{\mathbb{H}} X$ to be the restriction of id $\otimes\left(\phi^{\times}\right)^{*} \otimes\left(\psi^{\times}\right)^{*}$ to $U \otimes_{\mathbb{H}} V$. It follows trivially from the definitions that $\phi \otimes_{\mathbb{H}} \psi$ is $\mathbb{H}$-linear and satisfies $\left(\phi \otimes_{\mathbb{H}} \psi\right)\left(\left(U \otimes_{\mathbb{H}} V\right)^{\prime}\right) \subset\left(W \otimes_{\mathbb{H}} X\right)^{\prime}$. Thus $\phi \otimes_{\mathbb{H}} \psi$ is an AHI-morphism from $U \otimes_{\mathbb{H}} V$ to $W \otimes_{\mathbb{H}} X$. This is the quaternionic tensor product of $\phi$ and $\psi$.

Lemma 1.1.8 Suppose that $\phi: U \rightarrow W$ and $\psi: V \rightarrow X$ are injective AHImorphisms. Then $\phi \otimes_{\mathbb{H}} \psi: U \otimes_{\mathbb{H}} V \rightarrow W \otimes_{\mathbb{H}} X$ is an injective AHI-morphism.

Proof. Consider the map id $\otimes\left(\phi^{\times}\right)^{*} \otimes\left(\psi^{\times}\right)^{*}$ of (3). Clearly this maps $\iota_{U}(U) \otimes$ $\left(V^{\dagger}\right)^{*}$ to $\iota_{W}(W) \otimes\left(X^{\dagger}\right)^{*}$. As $\iota_{U}(U) \cong U$ and $\iota_{W}(W) \cong W$ and the map $\phi: U \rightarrow W$ is injective, we see that the kernel of id $\otimes\left(\phi^{\times}\right)^{*} \otimes\left(\psi^{\times}\right)^{*}$ on $\iota_{U}(U) \otimes\left(V^{\dagger}\right)^{*}$ is $\iota_{U}(U) \otimes \operatorname{Ker}\left(\psi^{\times}\right)^{*}$. Similarly, the kernel on $\left(U^{\dagger}\right)^{*} \otimes \iota_{V}(V)$ is $\operatorname{Ker}\left(\phi^{\times}\right)^{*} \otimes \iota_{V}(V)$. Thus the kernel of $\phi \otimes_{\mathbb{H}} \psi$ is

$$
\operatorname{Ker}\left(\phi \otimes_{\mathbb{H}} \psi\right)=\left(\iota_{U}(U) \otimes \operatorname{Ker}\left(\psi^{\times}\right)^{*}\right) \cap\left(\operatorname{Ker}\left(\phi^{\times}\right)^{*} \otimes \iota_{V}(V)\right) .
$$

But this is contained in $\left(\iota_{U}(U) \cap\left(\mathbb{H} \otimes \operatorname{Ker}\left(\phi^{\times}\right)^{*}\right)\right) \otimes\left(V^{\dagger}\right)^{*}$. Now $\iota_{U}(U) \cap$ $\left(\mathbb{H} \otimes \operatorname{Ker}\left(\phi^{\times}\right)^{*}\right)=0$, since if $\iota(u)$ lies in $\mathbb{H} \otimes \operatorname{Ker}\left(\phi^{\times}\right)^{*}$ then $\phi(u)=0$ in $W$, so $u=0$ as $\phi$ is injective. Thus $\operatorname{Ker}\left(\phi \otimes_{\mathbb{H}} \psi\right)=0$, and $\phi \otimes_{\mathbb{H}} \psi$ is injective.

The following lemma is trivial to prove. 
Lemma 1.1.9 Let $U, V$ be AHI-modules, and let $u \in U$ and $v \in V$ be nonzero. Suppose that $\alpha(u) \beta(v)=\beta(v) \alpha(u) \in \mathbb{H}$ for every $\alpha \in U^{\dagger}$ and $\beta \in V^{\dagger}$. Define an element $u \otimes_{\mathbb{H}} v$ of $\mathbb{H} \otimes\left(U^{\dagger}\right)^{*} \otimes\left(V^{\dagger}\right)^{*}$ by $\left(u \otimes_{\mathbb{H}} v\right) \cdot(\alpha \otimes \beta)=$ $\alpha(u) \beta(v) \in \mathbb{H}$. Then $u \otimes_{\mathbb{H}} v$ is a nonzero element of $U \otimes_{\mathbb{H}} V$.

The philosophy of the algebraic side of this paper is that much algebra that works over a commutative field such as $\mathbb{R}$ or $\mathbb{C}$ also has a close analogue over $\mathbb{H}$ (or some other noncommutative algebra), when we replace vector spaces over $\mathbb{R}$ or $\mathbb{C}$ by $A \mathbb{H}$-modules, and tensor products of vector spaces by the quaternionic tensor product $\otimes_{\mathbb{H}}$. Lemmas 1.1.5, 1.1.8 and 1.1.9 are examples of this philosophy, as they show that essential properties of the usual tensor product also hold for the quaternionic tensor product.

However, the quaternionic tensor product also has properties that are very unlike the usual tensor product, which come from the noncommutativity of the quaternions. For example, let $U, V$ be $\mathbb{H}$-modules, and let $U^{\prime}=V^{\prime}=\{0\}$. Then $U^{\dagger}=U^{\times}$and $V^{\dagger}=V^{\times}$. Suppose that $x \in U \otimes_{\mathbb{H}} V$, and let $p, q \in \mathbb{H}$, $\alpha \in U^{\dagger}$ and $\beta \in V^{\dagger}$. Then

$$
\begin{aligned}
x(\alpha \otimes \beta) \bar{p} \bar{q} & =x((p \cdot \alpha) \otimes \beta) \bar{q}=x((p \cdot \alpha) \otimes(q \cdot \beta)) \\
& =x(\alpha \otimes(q \cdot \beta)) \bar{p}=x(\alpha \otimes \beta) \bar{q} \bar{p},
\end{aligned}
$$

where we use the facts that $U^{\dagger}, V^{\dagger}$ are closed under the $\mathbb{H}$-action on $U^{\times}$, and the definition (11) of $U \otimes_{\mathbb{H}} V$. Choosing $p$ and $q$ such that $\bar{p} \bar{q} \neq \bar{q} \bar{p}$, equation (4) Shows that $x(\alpha \otimes \beta)=0$. Since this holds for all $\alpha, \beta, x=0$. Thus $U \otimes_{\mathbb{H}} V=\{0\}$. We have shown that the quaternionic tensor product of two nonzero AHI-modules can be zero.

Suppose that $U \cong \mathbb{H}^{k}$. Then the condition in Definition 1.1.1 implies that $\operatorname{dim}\left(U^{\dagger}\right) \geq k$. But $\operatorname{dim}\left(U^{\prime}\right)+\operatorname{dim}\left(U^{\dagger}\right)=4 k$, so $\operatorname{dim}\left(U^{\prime}\right) \leq 3 k$. The example above illustrates the general principle that if $\operatorname{dim}\left(U^{\prime}\right)$ is small, then quaternionic tensor products involving $U$ tend to be small or zero. A good rule is that the most interesting $\mathrm{A} \mathbb{H}$-modules $U$ are those in the range $2 k \leq$ $\operatorname{dim}\left(U^{\prime}\right) \leq 3 k$.

Here are some differences between the quaternionic and ordinary tensor products.

- In contrast to Lemma 1.1.9, if $u \in U$ and $v \in V$, there is in general no element ' $u \otimes_{\mathbb{H}} v$ ' in $U \otimes_{\mathbb{H}} V$. At best, there is a linear map from some vector subspace of $U \otimes V$ to $U \otimes_{\mathbb{H}} V$. 
- Suppose that $U, V$ are finite-dimensional AHH-modules, with $U \cong \mathbb{H}^{k}$, $V \cong \mathbb{H}^{l}$. It is easy to show that $U \otimes_{\mathbb{H}} V \cong \mathbb{H}^{n}$, for some integer $n$ with $0 \leq n \leq k l$. However, $n$ can vary discontinuously under smooth variations of $U^{\dagger}, V^{\dagger}$.

- If we wish, we can make $U^{\times}, V^{\times}$into AHI-modules. However, it is not in general true that $U^{\times} \otimes_{\mathbb{H}} V^{\times} \cong\left(U \otimes_{\mathbb{H}} V\right)^{\times}$, and there is no reason for $U^{\times} \otimes_{\mathbb{H}} V^{\times}$and $\left(U \otimes_{\mathbb{H}} V\right)^{\times}$even to have the same dimension. For this reason, dual AHI-modules seem not to be a very powerful tool.

- In contrast to Lemma 1.1.8, if $\phi: U \rightarrow W$ and $\psi: V \rightarrow X$ are surjective AHI-morphisms, then $\phi \otimes_{\mathbb{H}} \psi: U \otimes_{\mathbb{H}} V \rightarrow W \otimes_{\mathbb{H}} X$ does not have to be surjective. In particular, $U \otimes_{\mathbb{H}} V$ may be zero, but $W \otimes_{\mathbb{H}} X$ nonzero.

\subsection{Stable and semistable AHI-modules}

Now two special sorts of AHH-modules will be defined, called stable and semistable AHI-modules. Our aim in this paper has been to develop a strong analogy between the theories of AHI-modules and vector spaces over a field. For stable AHH-modules it turns out that this analogy is more complete than in the general case, because various important properties of the vector space theory hold for stable but not for general AHH-modules. Therefore, in applications of the theory it will often be useful to restrict to stable AHI-modules, to exploit their better behaviour. We begin with a definition.

Definition 1.2.1 Let $q \in \mathbb{I}$ be nonzero. Define an AHH-module $X_{q}$ by $X_{q}=\mathbb{H}$, and $X_{q}^{\prime}=\{p \in \mathbb{H}: p q=-q p\}$. Then $X_{q}^{\prime} \subset \mathbb{I}$ and $\operatorname{dim} X_{q}^{\prime}=2$. Let $\chi_{q}: X_{q} \rightarrow \mathbb{H}$ be the identity. Then $\chi_{q}\left(X_{q}^{\prime}\right) \subset \mathbb{I}=\mathbb{H}^{\prime}$, so $\chi_{q}$ is an A $\mathbb{H}-$ morphism. Suppose $U$ is any finite-dimensional AHI-module. Then $U \otimes_{\mathbb{H}} X_{q}$

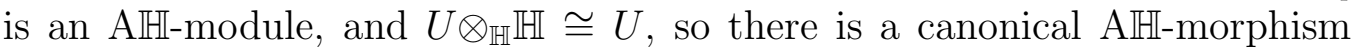
id $\otimes_{\mathbb{H}} \chi_{q}: U \otimes_{\mathbb{H}} X_{q} \rightarrow U$. We say that $U$ is a semistable $\mathrm{A} \mathbb{H}$-module if

$$
U=\left\langle\left(\operatorname{id} \otimes_{\mathbb{H}} \chi_{q}\right)\left(U \otimes_{\mathbb{H}} X_{q}\right): 0 \neq q \in \mathbb{I}\right\rangle,
$$

that is, $U$ is generated as an $\mathbb{H}$-module by the images $\operatorname{Im}\left(\mathrm{id} \otimes_{\mathbb{H}} \chi_{q}\right)$ for nonzero

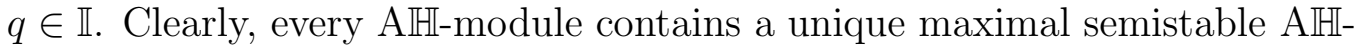
submodule, the submodule generated by the images $\operatorname{Im}\left(\mathrm{id} \otimes_{\mathbb{H}} \chi_{q}\right)$. 


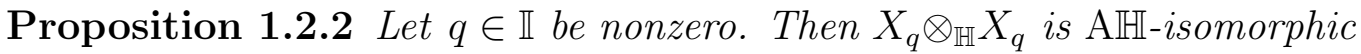

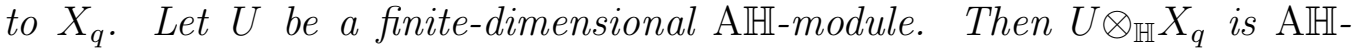
isomorphic to $n X_{q}$, the direct sum of $n$ copies of $X_{q}$, for some $n \geq 0$. Now suppose that $U$ is semistable, with $\operatorname{dim} U=4 j$ and $\operatorname{dim} U^{\prime}=2 j+r$, for integers $j, r$. Then $U \otimes_{\mathbb{H}} X_{q} \cong n X_{q}$, where $n \geq r$ for all nonzero $q \in \mathbb{I}$, and $n=r$ for generic $q \in \mathbb{I}$. Thus $r \geq 0$.

Proof. A short calculation shows that $X_{q} \otimes_{\mathbb{H}} X_{q} \cong X_{q}$ as AHH-modules. Let $U$ be a finite-dimensional AHI-module. Then id $\otimes_{\mathbb{H}} \chi_{q}: U \otimes_{\mathbb{H}} X_{q} \rightarrow U$ is an injective AHI-morphism, by Lemma 1.1.8. It is easy to show that $\left(U \otimes_{\mathbb{H}} X_{q}\right)^{\prime}$ is identified with $U^{\prime} \cap\left(q \cdot U^{\prime}\right)$ by id $\otimes_{\mathbb{H}} \chi_{q}$. Now $\langle 1, q\rangle \subset \mathbb{H}$ is a subalgebra $\mathbb{C}_{q}$ of $\mathbb{H}$ isomorphic to $\mathbb{C}$, and clearly $\left(U \otimes_{\mathbb{H}} X_{q}\right)^{\prime}$ is closed under $\mathbb{C}_{q}$. Choose a basis $u_{1}, \ldots, u_{n}$ of $\left(U \otimes_{\mathbb{H}} X_{q}\right)^{\prime}$ over the field $\mathbb{C}_{q}$. Suppose that $\Sigma q_{j} u_{j}=0$ in $U \otimes_{\mathbb{H}} X_{q}$, for $q_{1}, \ldots, q_{n} \in \mathbb{H}$. Let $p \in \mathbb{H}$ be nonzero, and such that $p q=-q p$. Then $\mathbb{H}$ splits as $\mathbb{H}=\mathbb{C}_{q} \oplus p \mathbb{C}_{q}$. Using this splitting, write $q_{j}=a_{j}+p b_{j}$, with $a_{j}, b_{j} \in \mathbb{C}_{q}$.

If $\alpha \in\left(U \otimes_{\mathbb{H}} X_{q}\right)^{\dagger}$, then $\alpha\left(u_{j}\right) \in p \mathbb{C}_{q}$, so $\alpha\left(\Sigma q_{j} u_{j}\right)=0$ implies that $\alpha\left(\Sigma a_{j} u_{j}\right)=\alpha\left(\Sigma b_{j} u_{j}\right)=0$. As this hold for all $\alpha \in\left(U \otimes_{\mathbb{H}} X_{q}\right)^{\dagger}$, we have $\Sigma a_{j} u_{j}=0$ and $\Sigma b_{j} u_{j}=0$. But $\left\{u_{j}\right\}$ is a basis over $\mathbb{C}_{q}$ and $a_{j}, b_{j} \in \mathbb{C}_{q}$. Thus $a_{j}=b_{j}=0$, and $q_{j}=0$. We have proved that $u_{1}, \ldots, u_{n}$ are linearly independent over $\mathbb{H}$. It is now easily shown that $\mathbb{H} \cdot u_{j} \cong X_{q}$, and that $U \otimes_{\mathbb{H}} X_{q}=\mathbb{H} \cdot u_{1} \oplus \cdots \oplus \mathbb{H} \cdot u_{n} \cong n X_{q}$, as we have to prove.

Now let $U$ be semistable, with $\operatorname{dim} U=4 j$ and $\operatorname{dim} U^{\prime}=2 j+r$. From above, $U^{\prime} \cap\left(q \cdot U^{\prime}\right)=\mathbb{C}_{q}^{n}$. But we have

$$
\begin{gathered}
\operatorname{dim}\left(U^{\prime} \cap\left(q \cdot U^{\prime}\right)\right)+\operatorname{dim}\left(U^{\prime}+q \cdot U^{\prime}\right) \\
\quad=\operatorname{dim} U^{\prime}+\operatorname{dim}\left(q \cdot U^{\prime}\right)=4 j+2 r .
\end{gathered}
$$

Since $U^{\prime}+q \cdot U^{\prime} \subset U, \operatorname{dim}\left(U^{\prime}+q \cdot U^{\prime}\right) \leq 4 j$, and so (5) shows that $2 n \geq 2 r$, with equality if and only if $U=U^{\prime}+q \cdot U^{\prime}$. Thus $n \geq r$ for all nonzero $q$, as we have to prove. To complete the proposition, it is enough to show that $U=U^{\prime}+q \cdot U^{\prime}$ for generic $q \in \mathbb{I}$.

As $U$ is semistable, it is generated by the images $\operatorname{Im}\left(\mathrm{id} \otimes_{\mathbb{H}} \chi_{q}\right)$. So suppose $U$ is generated by $\operatorname{Im}\left(\mathrm{id} \otimes_{\mathbb{H}} \chi_{q_{j}}\right)$ for $j=1, \ldots, k$, where $0 \neq q_{j} \in \mathbb{I}$. Let $q \in \mathbb{I}$, and suppose that $q q_{j} \neq q_{j} q$ for $j=1, \ldots, k$. This is true for generic $q$. Clearly $X_{q_{j}}^{\prime}+q \cdot X_{q_{j}}^{\prime}=X_{q_{j}}$, as $q q_{j} \neq q_{j} q$. Thus $\left(U \otimes_{\mathbb{H}} X_{q_{j}}\right)^{\prime}+q \cdot\left(U \otimes_{\mathbb{H}} X_{q_{j}}\right)^{\prime}=U \otimes_{\mathbb{H}} X_{q_{j}}$, as $U \otimes_{\mathbb{H}} X_{q_{j}} \cong n X_{q_{j}}$. We deduce that $\operatorname{Im}\left(\operatorname{id} \otimes_{\mathbb{H}} \chi_{q_{j}}\right)$ is contained in $U^{\prime}+q \cdot U^{\prime}$. 
But $U$ is generated by the spaces $\operatorname{Im}\left(\mathrm{id} \otimes_{\mathbb{H}} \chi_{q_{j}}\right)$, so $U=U^{\prime}+q \cdot U^{\prime}$. This completes the proof.

Now we can define stable AHI-modules.

Definition 1.2.3 Let $U$ be a finite-dimensional AHI-module. Then $\operatorname{dim} U=$ $4 j$ and $\operatorname{dim} U^{\prime}=2 j+r$, for some integers $j, r$. Define the virtual dimension of $U$ to be $r$. We say that $U$ is a stable AHI-module if $U$ is a semistable AHI-module, so that $r \geq 0$, and $U \otimes_{\mathbb{H}} X_{q} \cong r X_{q}$ for each nonzero $q \in \mathbb{I}$.

The point of this definition will become clear soon. Here are two propositions about stable and semistable AHI-modules.

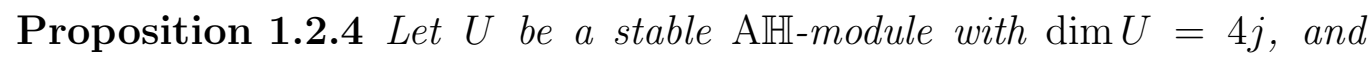
$\operatorname{dim} U^{\prime}=2 j+r$ for integers $j, r$. Let $V$ be a semistable AH-module with $\operatorname{dim} V=4 k$ and $\operatorname{dim} V^{\prime}=2 k+s$ for integers $k, s$. Then $\operatorname{dim}\left(U \otimes_{\mathbb{H}} V\right)=4 l$, where $l=j s+r k-r s$.

Proof. Regard $\mathbb{H} \otimes U^{\dagger} \otimes V^{\dagger}$ and $\mathbb{H} \otimes\left(U^{\dagger}\right)^{*} \otimes\left(V^{\dagger}\right)^{*}$ as $\mathbb{H}$-modules in the obvious way. Define a bilinear map $\Theta: \mathbb{H} \otimes\left(U^{\dagger}\right)^{*} \otimes\left(V^{\dagger}\right)^{*} \times \mathbb{H} \otimes U^{\dagger} \otimes V^{\dagger} \rightarrow \mathbb{H}$ by $\Theta(p \otimes \alpha \otimes \beta, q \otimes x \otimes y)=\alpha(x) \beta(y) p \bar{q}$ for $p, q \in \mathbb{H}, x \in U^{\dagger}, y \in V^{\dagger}$, $\alpha \in\left(U^{\dagger}\right)^{*}$ and $\beta \in\left(V^{\dagger}\right)^{*}$. Recall that $\iota_{U}(U) \otimes\left(V^{\dagger}\right)^{*}$ and $\left(U^{\dagger}\right)^{*} \otimes \iota_{V}(V)$ are $\mathbb{H}$-submodules of $\mathbb{H} \otimes\left(U^{\dagger}\right)^{*} \otimes\left(V^{\dagger}\right)^{*}$. Define a subspace $K_{U, V}$ of $\mathbb{H} \otimes U^{\dagger} \otimes V^{\dagger}$ by $z \in K_{U, V}$ if $\Theta(\zeta, z)=0$ whenever $\zeta \in \iota_{U}(U) \otimes\left(V^{\dagger}\right)^{*}$ or $\zeta \in\left(U^{\dagger}\right)^{*} \otimes \iota_{V}(V)$. Then $K_{U, V}$ is an $\mathbb{H}$-submodule of $\mathbb{H} \otimes U^{\dagger} \otimes V^{\dagger}$.

Now $\iota_{U}(U) \otimes\left(V^{\dagger}\right)^{*}+\left(U^{\dagger}\right)^{*} \otimes \iota_{V}(V)$ is an $\mathbb{H}$-submodule of $\mathbb{H} \otimes\left(U^{\dagger}\right)^{*} \otimes\left(V^{\dagger}\right)^{*}$, and clearly

$$
\begin{aligned}
\operatorname{dim} K_{U, V} & +\operatorname{dim}\left(\iota_{U}(U) \otimes\left(V^{\dagger}\right)^{*}+\left(U^{\dagger}\right)^{*} \otimes \iota_{V}(V)\right) \\
& =\operatorname{dim} \mathbb{H} \otimes\left(U^{\dagger}\right)^{*} \otimes\left(V^{\dagger}\right)^{*}=4(2 j-r)(2 k-s) .
\end{aligned}
$$

But $U \otimes_{\mathbb{H}} V=\left(\iota_{U}(U) \otimes\left(V^{\dagger}\right)^{*}\right) \cap\left(\left(U^{\dagger}\right)^{*} \otimes \iota_{V}(V)\right)$, and thus

$$
\begin{aligned}
\operatorname{dim}\left(U \otimes_{\mathbb{H}} V\right)= & \operatorname{dim}\left(\iota_{U}(U) \otimes\left(V^{\dagger}\right)^{*}\right)+\operatorname{dim}\left(\left(U^{\dagger}\right)^{*} \otimes \iota_{V}(V)\right) \\
& -\operatorname{dim}\left(\iota_{U}(U) \otimes\left(V^{\dagger}\right)^{*}+\left(U^{\dagger}\right)^{*} \otimes \iota_{V}(V)\right),
\end{aligned}
$$

so that $\operatorname{dim}\left(U \otimes_{\mathbb{H}} V\right)=4 j(2 k-s)+(2 j-r) 4 k-\left\{4(2 j-r)(2 k-s)-\operatorname{dim} K_{U, V}\right\}=$ $4 l+\operatorname{dim} K_{U, V}$, where $l=j s+r k-r s$. Therefore $\operatorname{dim}\left(U \otimes_{\mathbb{H}} V\right)=4 l$ if and only if $K_{U, V}=\{0\}$. 
Suppose that $W$ is an AH-module, and $\phi: W \rightarrow V$ is an AH-morphism. Then $\phi^{\times}: V^{\dagger} \rightarrow W^{\dagger}$, so that id $\otimes \phi^{\times}: \mathbb{H} \otimes U^{\dagger} \otimes V^{\dagger} \rightarrow \mathbb{H} \otimes U^{\dagger} \otimes W^{\dagger}$. We have $K_{U, V} \subset \mathbb{H} \otimes U^{\dagger} \otimes V^{\dagger}$ and $K_{U, W} \subset \mathbb{H} \otimes U^{\dagger} \otimes W^{\dagger}$. It is easy to show that $\left(\mathrm{id} \otimes \phi^{\times}\right)\left(K_{U, V}\right) \subset K_{U, W}$. Let $0 \neq q \in \mathbb{I}$, and put $W=V \otimes_{\mathbb{H}} X_{q}$, and $\phi=\mathrm{id} \otimes_{\mathbb{H}} \chi_{q}$. In this case $W \cong n X_{q}$. The argument above shows that $K_{U, X_{q}}=\{0\}$ if and only if $\operatorname{dim}\left(U \otimes_{\mathbb{H}} X_{q}\right)=4 r$. But by this holds by Definition 1.2.3, as $U$ is stable.

Thus $K_{U, X_{q}}=\{0\}$, and $K_{U, W}=\{0\}$ as $W \cong n X_{q}$. It follows that $\left(\mathrm{id} \otimes \phi^{\times}\right)\left(K_{U, V}\right)=\{0\}$, so $K_{U, V} \subset \mathbb{H} \otimes U^{\dagger} \otimes \operatorname{Ker} \phi^{\times}$. Now $V$ is semistable. Therefore $V$ is generated by submodules $\phi(W)$ of the above type, and the intersection of the subspaces $\operatorname{Ker} \phi^{\times} \subset V^{\dagger}$ for all nonzero $q$, must be zero. So $K_{U, V} \subset \mathbb{H} \otimes U^{\dagger} \otimes\{0\}$, giving $K_{U, V}=\{0\}$, and $\operatorname{dim}\left(U \otimes_{\mathbb{H}} V\right)=4 l$ from above, which completes the proof.

Proposition 1.2.5 Let $U$ be a stable AH-module, and $V$ a semistable AHmodule. Then $U \otimes_{\mathbb{H}} V$ is semistable.

Proof. Let $\operatorname{dim} U=4 j, \operatorname{dim} V=4 k, \operatorname{dim} U^{\prime}=2 j+r$ and $\operatorname{dim} V^{\prime}=2 k+s$. Then Proposition 1.2.4 shows that $\operatorname{dim}\left(U \otimes_{\mathbb{H}} V\right)=4 l$, where $l=j s+r k-r s$. Let $W \subset U \otimes_{\mathbb{H}} V$ be the AH-1-submodule of $U \otimes_{\mathbb{H}} V$ generated by the images $\operatorname{Im}\left(\mathrm{id} \otimes_{\mathbb{H}} \chi_{q}\right)$, where id $\otimes_{\mathbb{H}} \chi_{q}: U \otimes_{\mathbb{H}} V \otimes_{\mathbb{H}} X_{q} \rightarrow U \otimes_{\mathbb{H}} V$ and $0 \neq q \in \mathbb{I}$. Then $W$ is the maximal semistable AHI-submodule of $U \otimes_{\mathbb{H}} V$. We shall prove the proposition by explicitly constructing $l$ elements of $W$, that are linearly independent over $\mathbb{H}$. This will imply that $\operatorname{dim} W \geq 4 l$. Since $W \subset U \otimes_{\mathbb{H}} V$ and $\operatorname{dim}\left(U \otimes_{\mathbb{H}} V\right)=4 l$, we see that $W=U \otimes_{\mathbb{H}} V$, so $U \otimes_{\mathbb{H}} V$ is semistable.

Here is some new notation. Let $0 \neq q \in \mathbb{I}$, and define $U_{q}=\{u \in$ $U: \alpha(u) \in\langle 1, q\rangle$ for all $\left.\alpha \in U^{\dagger}\right\}$. Similarly define $V_{q},\left(U \otimes_{\mathbb{H}} V\right)_{q}$. It can be shown that $\mathbb{H} \cdot U_{q}=\operatorname{Im}\left(\mathrm{id} \otimes_{\mathbb{H}} \chi_{q}\right)$, and similarly for $V_{q},\left(U \otimes_{\mathbb{H}} V\right)_{q}$. Thus $\left(U \otimes_{\mathbb{H}} V\right)_{q} \subset W$. Since $V$ is semistable, we can choose nonzero elements $q_{1}, \ldots, q_{k} \in \mathbb{I}$ and $v_{1}, \ldots, v_{k} \in V$, such that $v_{a} \in V_{q_{a}}$ and $\left(v_{1}, \ldots, v_{k}\right)$ is a basis for $V$ over $\mathbb{H}$. Since $U$ is stable, $U_{q} \cong \mathbb{C}^{r}$ for each $0 \neq q \in \mathbb{I}$. Therefore for each $a=1, \ldots, k$ we may choose elements $u_{a 1}, \ldots, u_{a r}$ of $U$, such that $u_{a b} \in U_{q_{a}}$ and $u_{a 1}, \ldots, u_{a r}$ are linearly independent over $\mathbb{H}$ in $U$.

As $U$ is stable, it is not difficult to see that there are nonzero elements $p_{1}, \ldots, p_{j-r} \in \mathbb{I}$ and $u_{1}, \ldots, u_{j-r} \in U$, such that $u_{c} \in U_{p_{c}}$, and for each $a=1, \ldots, k$, the set $u_{a 1}, \ldots, u_{a r}, u_{1}, \ldots, u_{j-r}$ is linearly independent over $\mathbb{H}$. This is just a matter of picking generic elements $p_{c}$ and $u_{c}$, and showing that generically, linear independence holds. 
We shall also need another property. Define $F \subset U^{\dagger}$ by $F=\left\{\alpha \in U^{\dagger}\right.$ : $\alpha\left(u_{c}\right)=0$ for $\left.c=1, \ldots, j-r\right\}$. Now as $\alpha\left(u_{c}\right) \in\left\langle 1, q_{c}\right\rangle$ for each $\alpha \in U^{\dagger}$, the codimension of $F$ in $U^{\dagger}$ is at most $2(j-r)$. Since $\operatorname{dim} U^{\dagger}=2 j-r$, this gives $\operatorname{dim} F \geq r$. The second property we need is that for each $a=1, \ldots, k$, if $u \in\left\langle u_{a 1}, \ldots, u_{a r}\right\rangle_{\mathbb{H}}$ and $\alpha(u)=0$ for all $\alpha \in F$, then $u=0$. Here $\langle,\rangle_{\mathbb{H}}$ means the linear span over $\mathbb{H}$. Again, it can be shown that for generic choice of $p_{c}, v_{c}$, this property holds.

Now for $a=1, \ldots, k$ and $b=1, \ldots, r, u_{a b} \in U_{q_{a}}$ and $v_{a} \in V_{q_{a}}$. Lemma 1.1 .9 gives an element $u_{a b} \otimes_{\mathbb{H}} v_{a}$ in $U \otimes_{\mathbb{H}} V$. Moreover $u_{a b} \otimes_{\mathbb{H}} v_{a} \in\left(U \otimes_{\mathbb{H}} V\right)_{q_{a}}$. Thus $u_{a b} \otimes_{\mathbb{H}} v_{a} \in W$. Therefore, we have made $k r$ elements $u_{a b} \otimes_{\mathbb{H}} v_{a}$ of $W$. Similarly, for $c=1, \ldots, j-r$ and $d=1, \ldots, s$, the elements $u_{c} \otimes_{\mathbb{H}} v_{c d}$ exist in $W$, which gives a further $(j-r) s$ elements of $W$. Since $k r+(j-r) s=l$, we have constructed $l$ explicit elements $u_{a b} \otimes_{\mathbb{H}} v_{a}$ and $u_{c} \otimes_{\mathbb{H}} v_{c d}$ of $W$.

Suppose that $\Sigma_{a, b} x_{a b} u_{a b} \otimes_{\mathbb{H}} v_{a}+\Sigma_{c, d} y_{c d} u_{c} \otimes_{\mathbb{H}} v_{c d}=0$ in $U \otimes_{\mathbb{H}} V$, where $x_{a b} \in \mathbb{H}$ for $a=1, \ldots, k, b=1, \ldots, r$ and $y_{c d} \in \mathbb{H}$ for $c=1, \ldots, j-r$ and $d=1, \ldots, s$. We shall show that $x_{a b}=y_{c d}=0$. Now this equation implies that

$$
\sum_{a, b} x_{a b} \alpha\left(u_{a b}\right) \beta\left(v_{a}\right)+\sum_{c, d} y_{c d} \alpha\left(u_{c}\right) \beta\left(v_{c d}\right)=0
$$

for all $\alpha \in U^{\dagger}$ and $\beta \in V^{\dagger}$, by Lemma 1.1.9. Let $\alpha \in F$. Then $\alpha\left(u_{c}\right)=0$, so $\Sigma_{a, b} x_{a b} \alpha\left(u_{a b}\right) \beta\left(v_{a}\right)=0$. As this holds for all $\beta \in V^{\dagger}$ and the $v_{a}$ are linearly independent over $\mathbb{H}$, it follows that $\Sigma_{b} x_{a b} \alpha\left(u_{a b}\right)=0$ for all $a$ and all $\alpha \in F$.

By the property of $F$ assumed above, this implies that $x_{a b}=0$ for all $a, b$. It is now easy to show that $y_{c d}=0$ for all $c, d$. Thus the $l$ elements $u_{a b} \otimes_{\mathbb{H}} v_{a}$ and $u_{c} \otimes_{\mathbb{H}} v_{c d}$ of $W$ are linearly independent over $\mathbb{H}$, so $\operatorname{dim} W \geq 4 l$. But $\operatorname{dim}\left(U \otimes_{\mathbb{H}} V\right)=4 l$ by Proposition 1.2.4. So $U \otimes_{\mathbb{H}} V=W$, and $U \otimes_{\mathbb{H}} V$ is semistable. This finishes the proof.

We can now prove the main result of this section.

Theorem 1.2.6 Let $U$ be a stable AHH-module and $V$ be a semistable AHImodule with

$$
\operatorname{dim} U=4 j, \operatorname{dim} U^{\prime}=2 j+r, \operatorname{dim} V=4 k \text { and } \operatorname{dim} V^{\prime}=2 k+s
$$

for integers $j, k, r$ and $s$. Then $U \otimes_{\mathbb{H}} V$ is a semistable AH-module with $\operatorname{dim}\left(U \otimes_{\mathbb{H}} V\right)=4 l$ and $\operatorname{dim}\left(U \otimes_{\mathbb{H}} V\right)^{\prime}=2 l+t$, where $l=j s+r k-r s$ and $t=r s$. If $V$ is stable, then $U \otimes_{\mathbb{H}} V$ is stable. 
Proof. Let $l=j s+r k-r s$ and $t=r s$. Proposition 1.2.4 shows that $\operatorname{dim}\left(U \otimes_{\mathbb{H}} V\right)=4 l$. As $U$ is stable, $U \otimes_{\mathbb{H}} X_{q} \cong r X_{q}$ for nonzero $q \in \mathbb{I}$. As $V$ is semistable, Proposition 1.2 .2 shows that $V \otimes_{\mathbb{H}} X_{q} \cong s X_{q}$ for generic $q \in \mathbb{I}$. Thus $U \otimes_{\mathbb{H}} V \otimes_{\mathbb{H}} X_{q} \cong r s X_{q}=t X_{q}$ for generic $q \in \mathbb{I}$. Also, Proposition 1.2.5 shows that $U \otimes_{\mathbb{H}} V$ is semistable. Combining these two facts with Proposition 1.2.2, we see that $\operatorname{dim}\left(U \otimes_{\mathbb{H}} V\right)^{\prime}=2 l+t$, as we have to prove.

It remains to show that if $V$ is stable, then $U \otimes_{\mathbb{H}} V$ is stable. Suppose $V$ is stable. Then $V \otimes_{\mathbb{H}} X_{q} \cong s X_{q}$ for all nonzero $q \in \mathbb{I}$, so $U \otimes_{\mathbb{H}} V \otimes_{\mathbb{H}} X_{q}=t X_{q}$ for all nonzero $q \in \mathbb{I}$. As $U \otimes_{\mathbb{H}} V$ is semistable, it is stable, by Definition 1.2.3. This completes the proof.

Theorem 1.2.6 shows that if $U$ is stable and $V$ semistable, then the virtual dimension of $U \otimes_{\mathbb{H}} V$ is the product of the virtual dimensions of $U$ and $V$. Thus the virtual dimension is a good analogue of the dimension of a vector space, as it multiplies under $\otimes_{\mathbb{H}}$. Note also that $(j-r) / r+(k-s) / s=(l-t) / t$, so that the nonnegative function $U \mapsto(j-r) / r$ behaves additively under $\otimes_{\mathbb{H}}$.

Next we will show that generic AHI-modules $\left(U, U^{\prime}\right)$ with positive virtual dimension are stable. Thus there are many stable AHI-modules.

Lemma 1.2.7 Let $j, r$ be integers with $0<r \leq j$. Let $U=\mathbb{H}^{j}$, and let $U^{\prime}$ be a real vector subspace of $U$ with $\operatorname{dim} U^{\prime}=2 j+r$. Then for generic subspaces $U^{\prime},\left(U, U^{\prime}\right)$ is a stable $\mathrm{A} \mathbb{H}$-module.

Proof. Let $G$ be the Grassmannian of real $(2 j+r)$-planes in $U \cong \mathbb{R}^{4 j}$. Then $U^{\prime} \in G$, and $\operatorname{dim} G=4 j^{2}-r^{2}$. The condition for $\left(U, U^{\prime}\right)$ to be an AHI-module is that $\mathbb{H} \cdot U^{\dagger}=U^{\times}$. A calculation shows that this fails for a subset of $G$ of codimension $4(j-r+1)$. Thus for generic $U^{\prime} \in G,\left(U, U^{\prime}\right)$ is an AHI-module.

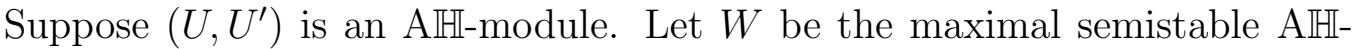
submodule in $U$. Then $W \cong \mathbb{H}^{k}$, for some $k$ with $r \leq k \leq j$. A calculation shows that for given $k$, the subset of $G$ with $W \cong \mathbb{H}^{k}$ is of codimension $2(j-k) r$. Thus for generic $U^{\prime} \in G, W=\mathbb{H}^{j}=U$, and $U$ is semistable.

Suppose $\left(U, U^{\prime}\right)$ is semistable. Let $0 \neq q \in \mathbb{I}$. Then $U \otimes_{\mathbb{H}} X_{q} \cong r X_{q}$ if and only if $U^{\prime} \cap q \cdot U^{\prime}=\mathbb{R}^{2 r}$. A computation shows that this fails for a subset of $G$ of codimension $2 r+2$. Thus the condition $U^{\prime} \cap q \cdot U^{\prime}=\mathbb{R}^{2 r}$ for all nonzero $q \in \mathbb{I}$ fails for a subset of $G$ of codimension at most $2 r$, since this subset is the union of a 2-dimensional family of $2 r+2$-codimensional subsets, the 2-dimensional family being $\mathcal{S}^{2}$, the unit sphere in $\mathbb{I}$. Therefore for generic $U^{\prime} \in G, U \otimes_{\mathbb{H}} X_{q} \cong r X_{q}$ for all nonzero $q \in \mathbb{I}$, and $U$ is stable. 
We leave the proof of this proposition to the reader, as a (difficult) exercise.

Proposition 1.2.8 Let $U$ be a stable $\mathrm{AHH}-m o d u l e$, with $\operatorname{dim} U=4 j$ and $\operatorname{dim} U^{\prime}=2 j+r$. Let $n$ be a positive integer. Then $S_{\mathbb{H}}^{n} U$ and $\Lambda_{\mathbb{H}}^{n} U$ are stable $\mathrm{A} \mathbb{H}$-modules, with $\operatorname{dim}\left(S_{\mathbb{H}}^{n} U\right)=4 k, \operatorname{dim}\left(S_{\mathbb{H}}^{n} U\right)^{\prime}=2 k+s, \operatorname{dim}\left(\Lambda_{\mathbb{H}}^{n} U\right)=4 l$ and $\operatorname{dim}\left(\Lambda_{\mathbb{H}}^{n} U\right)^{\prime}=2 l+t$, where

$$
\begin{array}{ll}
k=(j-r)\left(\begin{array}{c}
r+n-1 \\
n-1
\end{array}\right)+\left(\begin{array}{c}
r+n-1 \\
n
\end{array}\right), \quad s=\left(\begin{array}{c}
r+n-1 \\
n
\end{array}\right), \\
l=(j-r)\left(\begin{array}{l}
r-1 \\
n-1
\end{array}\right)+\left(\begin{array}{l}
r \\
n
\end{array}\right) \quad \text { and } & t=\left(\begin{array}{l}
r \\
n
\end{array}\right) .
\end{array}
$$

\subsection{Stable AHI-modules and exact sequences}

Recall that if $U, V, W$ are vector spaces and $\phi: U \rightarrow V, \psi: V \rightarrow W$ are linear maps, then we say that the sequence $U \stackrel{\phi}{\rightarrow} V \stackrel{\psi}{\rightarrow} W$ is exact at $V$ if $\operatorname{Im} \phi=\operatorname{Ker} \psi$. Here is the analogue of this for AHI-modules.

Definition 1.3.1 Let $U, V, W$ be AHI-modules, and let $\phi: U \rightarrow V$ and $\psi: V \rightarrow W$ be AHI-morphisms. We say that the sequence $U \stackrel{\phi}{\rightarrow} V \stackrel{\psi}{\rightarrow} W$ is $\mathrm{AH}$-exact at $V$ if the sequence $U^{\phi} \rightarrow \stackrel{\psi}{\rightarrow} W$ is exact at $V$, and the sequence $U^{\prime} \stackrel{\phi}{\rightarrow} V^{\prime} \stackrel{\psi}{\rightarrow} W^{\prime}$ is exact at $V^{\prime}$. We say that a sequence of AHI-morphisms is $\mathrm{A} \mathbb{H}$-exact if it is $\mathrm{AH}$-exact at every term.

Here is an example of some bad behaviour of the theory.

Example 1.3.2 Define $U=\mathbb{H}$ and $U^{\prime}=\{0\}$. Define $V=\mathbb{H}^{2}$ and $V^{\prime}=$ $\left\langle\left(1, i_{1}\right),\left(1, i_{2}\right)\right\rangle$. Define $W=\mathbb{H}$ and $W^{\prime}=\left\langle i_{1}, i_{2}\right\rangle$. Then $U, V, W$ are AHImodules. Define linear maps $\phi: U \rightarrow V$ by $\phi(q)=(q, 0)$, and $\psi: V \rightarrow W$ by $\psi((p, q))=q$. Then $\phi, \psi$ are $\mathrm{AHI}$-morphisms, and the sequence $0 \rightarrow$ $U \stackrel{\phi}{\rightarrow} V \stackrel{\psi}{\rightarrow} W \rightarrow 0$ is AHI-exact.

Now set $Z$ to be the AHI-module $W$. A short calculation shows that $U \otimes_{\mathbb{H}} Z=\{0\}, V \otimes_{\mathbb{H}} Z=\{0\}$, but $W \otimes_{\mathbb{H}} Z \cong W$. It follows that the sequence

$$
0 \rightarrow U \otimes_{\mathbb{H}} Z \stackrel{\phi \otimes_{\mathbb{H}}^{\text {id }}}{\longrightarrow} V \otimes_{\mathbb{H}} Z \stackrel{\psi \otimes_{\mathbb{H}} \text { id }}{\longrightarrow} W \otimes_{\mathbb{H}} Z \rightarrow 0
$$


is not $\mathrm{AH}$-exact at $W \otimes_{\mathbb{H}} Z$. This contrasts with the behaviour of exact sequences of real vector spaces under the tensor product.

The following proposition gives a clearer idea of what is happening.

Proposition 1.3.3 Suppose that $U, V, W$ and $Z$ are finite-dimensional AHmodules, that $\phi: U \rightarrow V$ and $\psi: V \rightarrow W$ are AHI-morphisms, and that the sequence $0 \rightarrow U \stackrel{\phi}{\rightarrow} V \stackrel{\psi}{\rightarrow} W \rightarrow 0$ is $\mathrm{AH}$-exact. Then the sequence

$$
0 \rightarrow W^{\dagger} \stackrel{\psi^{\times}}{\longrightarrow} V^{\dagger} \stackrel{\phi^{\times}}{\longrightarrow} U^{\dagger} \rightarrow 0
$$

is exact, and the sequence

$$
0 \rightarrow U \otimes_{\mathbb{H}} Z \stackrel{\phi \otimes_{\mathbb{H}} \text { id }}{\longrightarrow} V \otimes_{\mathbb{H}} Z \stackrel{\psi \otimes_{\mathbb{H}} \text { id }}{\longrightarrow} W \otimes_{\mathbb{H}} Z \rightarrow 0
$$

is $\mathrm{A} \mathbb{H}$-exact at $U \otimes_{\mathbb{H}} Z$ and $V \otimes_{\mathbb{H}} Z$, but it need not be $\mathrm{A} \mathbb{H}$-exact at $W \otimes_{\mathbb{H}} Z$.

Proof. Since $\psi: V \rightarrow W$ is surjective, $\psi^{\times}: W^{\times} \rightarrow V^{\times}$is injective, and thus $\psi^{\times}: W^{\dagger} \rightarrow V^{\dagger}$ is injective. Now $\phi$ induces a map $\phi: U / U^{\prime} \rightarrow V / V^{\prime}$. Suppose that $u+U^{\prime}$ lies in the kernel of this map. Then $\phi(u) \in V^{\prime}$. By exactness, $\psi(\phi(u))=0$. As the sequence $U^{\prime} \rightarrow V^{\prime} \rightarrow W^{\prime}$ is also exact, $\phi(u)=\phi\left(u^{\prime}\right)$ for some $u^{\prime} \in U^{\prime}$. But $\phi$ is injective, so $u \in U^{\prime}$. Thus the map $\phi: U / U^{\prime} \rightarrow V / V^{\prime}$ is injective. It follows that the map $\phi^{*}:\left(V / V^{\prime}\right)^{*} \rightarrow\left(U / U^{\prime}\right)^{*}$ is surjective.

Since $U, V$ are finite-dimensional, it follows that $U^{\dagger} \cong\left(U / U^{\prime}\right)^{*}$ and $V^{\dagger} \cong$ $\left(V / V^{\prime}\right)^{*}$. Under these isomorphisms $\phi^{*}$ is identified with $\phi^{\times}$. Thus $\phi^{\times}$: $V^{\dagger} \rightarrow U^{\dagger}$ is surjective. Using exactness we see that $\operatorname{dim} V=\operatorname{dim} U+\operatorname{dim} W$ and $\operatorname{dim} V^{\prime}=\operatorname{dim} U^{\prime}+\operatorname{dim} W^{\prime}$. By subtraction we find that $\operatorname{dim} V^{\dagger}=$ $\operatorname{dim} U^{\dagger}+\operatorname{dim} W^{\dagger}$. But we have already shown that $\psi^{\times}: W^{\dagger} \rightarrow V^{\dagger}$ is injective, and $\phi^{\times}: V^{\dagger} \rightarrow U^{\dagger}$ is surjective. Using these facts, we see that the sequence (7) is exact, as we have to prove.

Now $\phi: U \rightarrow V$ is injective, and clearly id :Z $Z Z$ is injective. Thus Lemma 1.1 .8 shows that $\phi \otimes_{\mathbb{H}}$ id $: U \otimes_{\mathbb{H}} Z \rightarrow V \otimes_{\mathbb{H}} Z$ is injective. So the sequence (8) is $A \mathbb{H}$-exact at $U \otimes_{\mathbb{H}} Z$, as we have to prove. Suppose that $x \in \operatorname{Ker}\left(\psi \otimes_{\mathbb{H}} \mathrm{id}\right)$. It is easy to show, using exactness, injectivity, and the definition of $\otimes_{\mathbb{H}}$, that $x \in \operatorname{Im}\left(\phi \otimes_{\mathbb{H}} \mathrm{id}\right)$. Thus $\operatorname{Ker}\left(\psi \otimes_{\mathbb{H}} \mathrm{id}\right)=\operatorname{Im}\left(\phi \otimes_{\mathbb{H}} \mathrm{id}\right)$, and the sequence (8) is exact at $V \otimes_{\mathbb{H}} Z$, as we have to prove.

Recall from Definition 1.1.4 that as $U, Z$ are finite-dimensional, $\lambda_{U, Z}$ is surjective. Suppose that $a \in\left(U \otimes_{\mathbb{H}} Z\right)^{\dagger}$. Then $a=\lambda_{U, Z}(b)$ for some $b \in U^{\dagger} \otimes$ 
$Z^{\dagger}$. The map $\phi^{\times}: V^{\dagger} \rightarrow U^{\dagger}$ is surjective from above, and thus $b=\left(\phi^{\times} \otimes \mathrm{id}\right)(c)$ for some $c \in V^{\dagger} \otimes Z^{\dagger}$. Thus $a=\lambda_{U, Z} \circ\left(\phi^{\times} \otimes \mathrm{id}\right)(c)$. But it is easy to see that $\lambda_{U, Z} \circ\left(\phi^{\times} \otimes \mathrm{id}\right)=\left(\phi \otimes_{\mathbb{H}} \mathrm{id}\right)^{\times} \circ \lambda_{V, Z}$, as maps $V^{\dagger} \otimes Z^{\dagger} \rightarrow\left(U \otimes_{\mathbb{H}} Z\right)^{\dagger}$. Thus if $a \in\left(U \otimes_{\mathbb{H}} Z\right)^{\dagger}$, then $a=\left(\phi \otimes_{\mathbb{H}} \mathrm{id}\right)^{\times}(d)$, for $d=\lambda_{V, Z}(c) \in\left(V \otimes_{\mathbb{H}} Z\right)^{\dagger}$. Therefore $\left(\phi \otimes_{\mathbb{H}} \mathrm{id}\right)^{\times}:\left(V \otimes_{\mathbb{H}} Z\right)^{\dagger} \rightarrow\left(U \otimes_{\mathbb{H}} Z\right)^{\dagger}$ is surjective.

Let $x \in\left(V \otimes_{\mathbb{H}} Z\right)^{\prime}$, and suppose $\left(\psi \otimes_{\mathbb{H}} \mathrm{id}\right)(x)=0$. As $(8)$ is exact, $x=$ $\left(\phi \otimes_{\mathbb{H}} \mathrm{id}\right)(y)$, and as $\phi \otimes_{\mathbb{H}}$ id is injective, $y$ is unique. We shall show that $y \in\left(U \otimes_{\mathbb{H}} Z\right)^{\prime}$. It is enough to show that for each $\alpha \in\left(U \otimes_{\mathbb{H}} Z\right)^{\dagger}, \alpha(y) \in \mathbb{I}$. Since $\left(\phi \otimes_{\mathbb{H}} \mathrm{id}\right)^{\times}:\left(V \otimes_{\mathbb{H}} Z\right)^{\dagger} \rightarrow\left(U \otimes_{\mathbb{H}} Z\right)^{\dagger}$ is surjective, $\alpha=\left(\phi \otimes_{\mathbb{H}} \mathrm{id}\right)^{\times}(\beta)$ for $\beta \in\left(V \otimes_{\mathbb{H}} Z\right)^{\dagger}$. Then $\alpha(y)=\beta(x)$. But $x \in\left(V \otimes_{\mathbb{H}} Z\right)^{\prime}$ and $\beta \in\left(V \otimes_{\mathbb{H}} Z\right)^{\dagger}$, so $\beta(x) \in \mathbb{I}$. Thus $\alpha(y) \in \mathbb{I}$, and $y \in\left(U \otimes_{\mathbb{H}} Z\right)^{\prime}$. It follows that the sequence $\left(U \otimes_{\mathbb{H}} Z\right)^{\prime} \stackrel{\phi \otimes_{\mathbb{H}} \text { id }}{\longrightarrow}\left(V \otimes_{\mathbb{H}} Z\right)^{\prime} \stackrel{\phi \otimes_{\mathbb{H}} \text { id }}{\longrightarrow}\left(W \otimes_{\mathbb{H}} Z\right)^{\prime}$ is exact at $\left(V \otimes_{\mathbb{H}} Z\right)^{\prime}$. Therefore (8) is AHI-exact at $V \otimes_{\mathbb{H}} Z$, as we have to prove. The example above shows that the sequence need not be $\mathrm{AH}-$-exact at $W \otimes_{\mathbb{H}} Z$, and the proposition is finished.

In the language of category theory, Proposition 1.3 .3 shows that when $Z$ is a finite-dimensional AIH-module, the operation $\otimes_{\mathbb{H}} Z$ is a left-exact functor, but may not be a right-exact functor. However, the next proposition shows that right-exactness does hold for stable and semistable AHI-modules.

Proposition 1.3.4 Suppose that $U, V, W$ are AH-modules, that $U$ and $W$ are stable, that $\phi: U \rightarrow V$ and $\psi: V \rightarrow W$ are $\mathrm{A} \mathbb{H}$-morphisms, and that the sequence $0 \rightarrow U \stackrel{\phi}{\rightarrow} V \stackrel{\psi}{\rightarrow} W \rightarrow 0$ is $\mathrm{AH}$-exact. Let $Z$ be a semistable AHImodule. Then the following sequence is AHI-exact:

$$
0 \rightarrow U \otimes_{\mathbb{H}} Z \stackrel{\phi \otimes_{\mathbb{H}} \text { id }}{\longrightarrow} V \otimes_{\mathbb{H}} Z \stackrel{\psi \otimes_{\mathbb{H}} \text { id }}{\longrightarrow} W \otimes_{\mathbb{H}} Z \rightarrow 0 .
$$

Proof. Let $\operatorname{dim} U=4 j, \operatorname{dim} V=4 k, \operatorname{dim} W=4 l, \operatorname{dim} U^{\prime}=2 j+r, \operatorname{dim} V^{\prime}=$ $2 k+s, \operatorname{dim} W^{\prime}=2 l+t, \operatorname{dim} Z=4 a$ and $\operatorname{dim} Z^{\prime}=2 a+b$. Then by Theorem 1.2.6 we have

$$
\begin{aligned}
\operatorname{dim}\left(U \otimes_{\mathbb{H}} Z\right) & =4(j b+r a-r b), \operatorname{dim}\left(W \otimes_{\mathbb{H}} Z\right)=4(l b+t a-t b), \\
\operatorname{dim}\left(U \otimes_{\mathbb{H}} Z\right)^{\prime} & =2 j b+2 r a-r b, \operatorname{dim}\left(W \otimes_{\mathbb{H}} Z\right)^{\prime}=2 l b+2 t a-t b .
\end{aligned}
$$

Theorem 1.2.6 calculates the dimensions of a quaternionic tensor product of stable and semistable AHI-modules. Examining the proof, it is easy to 
see that these dimensions are actually lower bounds for the dimensions of a quaternionic tensor product of general AHI-modules. Therefore

$$
\operatorname{dim}\left(V \otimes_{\mathbb{H}} Z\right) \geq 4(k b+s a-s b) \text { and } \operatorname{dim}\left(V \otimes_{\mathbb{H}} Z\right)^{\prime} \geq 2 k b+2 s a-s b .
$$

By Proposition 1.3.3, the sequence (9) is AHI-exact at $U \otimes_{\mathbb{H}} Z$ and $V \otimes_{\mathbb{H}} Z$. The only way AHI-exactness at $W \otimes_{\mathbb{H}} Z$ can fail is for $\psi \otimes_{\mathbb{H}}$ id $: V \otimes_{\mathbb{H}} Z \rightarrow$ $W \otimes_{\mathbb{H}} Z$ or $\psi \otimes_{\mathbb{H}}$ id $:\left(V \otimes_{\mathbb{H}} Z\right)^{\prime} \rightarrow\left(W \otimes_{\mathbb{H}} Z\right)^{\prime}$ not to be surjective. We deduce that

$$
\begin{array}{r}
\operatorname{dim}\left(U \otimes_{\mathbb{H}} Z\right)+\operatorname{dim}\left(W \otimes_{\mathbb{H}} Z\right) \geq \operatorname{dim}\left(V \otimes_{\mathbb{H}} Z\right), \\
\operatorname{dim}\left(U \otimes_{\mathbb{H}} Z\right)^{\prime}+\operatorname{dim}\left(W \otimes_{\mathbb{H}} Z\right)^{\prime} \geq \operatorname{dim}\left(V \otimes_{\mathbb{H}} Z\right)^{\prime}
\end{array}
$$

But $0 \rightarrow U \stackrel{\phi}{\rightarrow} V \stackrel{\psi}{\rightarrow} W \rightarrow 0$ is AHI-exact, so that $k=j+l$ and $s=r+t$. Combining (10), (11) and (12), we see that equality holds in (11) and (12), because the inequalities go opposite ways. Counting dimensions, $\psi \otimes_{\mathbb{H}}$ id : $V \otimes_{\mathbb{H}} Z \rightarrow W \otimes_{\mathbb{H}} Z$ and $\psi \otimes_{\mathbb{H}}$ id $:\left(V \otimes_{\mathbb{H}} Z\right)^{\prime} \rightarrow\left(W \otimes_{\mathbb{H}} Z\right)^{\prime}$ must be surjective. Thus by definition, (9) is AHI-exact at $W \otimes_{\mathbb{H}} Z$, and the proposition is proved.

The proposition gives one reason why it is convenient, in many situations, to work with stable AHI-modules rather than general AHI-modules.

Proposition 1.3.5 Suppose $U, V, W$ are AHI-modules, $\phi: U \rightarrow V$ and $\psi$ : $V \rightarrow W$ are $\mathrm{AH}-m o r p h i s m s$, and that the sequence $0 \rightarrow U \stackrel{\phi}{\rightarrow} V \stackrel{\psi}{\rightarrow} W \rightarrow 0$ is $\mathrm{AH}$-exact. If $U$ and $W$ are stable $\mathrm{AH}-$ modules, then $V$ is a stable $\mathrm{AH}-$ module.

Proof. Let $q \in \mathbb{I}$ be nonzero, and apply Proposition 1.3.4 with $Z=X_{q}$, the semistable AHI-module defined in $\$ 1.2$. Let $\operatorname{dim} V=4 k$ and $\operatorname{dim} V^{\prime}=$ $2 k+s$ as in the proof of the proposition. Since equality holds in (11), $\operatorname{dim}\left(V \otimes_{\mathbb{H}} X_{q}\right)=4 s$, so that $V \otimes_{\mathbb{H}} X_{q} \cong s X_{q}$. But this is the main condition for $V$ to be stable. Thus, it remains only to show that $V$ is semistable.

Let $S$ be the maximal semistable AHI-submodule of $V$. As $U$ is semistable, $\phi(U) \subset S$. Also, from above the map $\psi \otimes_{\mathbb{H}}$ id $: V \otimes_{\mathbb{H}} X_{q} \rightarrow W \otimes_{\mathbb{H}} X_{q}$ is surjective. As $W$ is semistable, we deduce that $\psi(S)=W$. But $0 \rightarrow U \stackrel{\phi}{\rightarrow} V \stackrel{\psi}{\rightarrow} W \rightarrow$ 0 is A $\mathbb{H}$-exact, so $\phi(U) \subset S$ and $\psi(S)=W$ imply that $S=V$. Therefore $V$ is semistable, so $V$ is stable. 


\section{Algebraic structures over the quaternions}

In this chapter, the machinery of Chapter 1 will be used to define quaternionic analogues of various algebraic concepts. We shall only discuss those structures we shall need for our study of hypercomplex geometry, but the reader will soon see how the process works in general. First, in $\$ 2.1$ we define $\mathrm{H}$-algebras, the quaternionic version of commutative algebras, and modules over H-algebras. Then in $\$ 2.2$ we define HL-algebras and HP-algebras, the analogues of Lie algebras and Poisson algebras. Section 2.3 is about filtered and graded H-algebras, and $\$ 2.4$ considers free and finitely-generated $\mathrm{H}$-algebras.

\section{$2.1 \quad$ H-algebras and modules}

Now we will define the quaternionic version of a commutative algebra, which we shall call an $\mathrm{H}$-algebra, and also modules over $\mathrm{H}$-algebras. In Chapter 3 we shall see that the q-holomorphic functions on a hypercomplex manifold form an H-algebra. Here are two axioms.

Axiom A. $(i) A$ is an AHI-module.

(ii) There is an AHI-morphism $\mu_{A}: A \otimes_{\mathbb{H}} A \rightarrow A$, called the multiplication map.

(iii) $\Lambda_{\mathbb{H}}^{2} A \subset \operatorname{Ker} \mu_{A}$. Thus $\mu_{A}$ is commutative.

(iv) The AHI-morphisms $\mu_{A}: A \otimes_{\mathbb{H}} A \rightarrow A$ and id $: A \rightarrow A$ combine to give AHI-morphisms $\mu_{A} \otimes_{\mathbb{H}}$ id and id $\otimes_{\mathbb{H}} \mu_{A}: A \otimes_{\mathbb{H}} A \otimes_{\mathbb{H}} A \rightarrow A \otimes_{\mathbb{H}} A$. Composing with $\mu_{A}$ gives AHI-morphisms $\mu_{A} \circ\left(\mu_{A} \otimes_{\mathbb{H}}\right.$ id $)$ and $\mu_{A} \circ\left(\right.$ id $\left.\otimes_{\mathbb{H}} \mu_{A}\right)$ : $A \otimes_{\mathbb{H}} A \otimes_{\mathbb{H}} A \rightarrow A$. Then $\mu_{A} \circ\left(\mu_{A} \otimes_{\mathbb{H}} \mathrm{id}\right)=\mu_{A} \circ\left(\mathrm{id} \otimes_{\mathbb{H}} \mu_{A}\right)$. This is associativity of multiplication.

(v) An element $1 \in A$ called the identity is given, with $1 \notin A^{\prime}$ and $\mathbb{I} \cdot 1 \subset A^{\prime}$.

(vi) Part $(v)$ implies that if $\alpha \in A^{\dagger}$ then $\alpha(1) \in \mathbb{R}$. Thus for each $a \in A, 1 \otimes_{\mathbb{H}} a$ and $a \otimes_{\mathbb{H}} 1 \in A \otimes_{\mathbb{H}} A$ by Lemma 1.1.9. Then $\mu_{A}\left(1 \otimes_{\mathbb{H}} a\right)=\mu_{A}\left(a \otimes_{\mathbb{H}} 1\right)=a$ for each $a \in A$. Thus 1 is a multiplicative identity.

Axiom M. $(i)$ Let $U$ be an AHI-module.

(ii) There is an AHI-morphism $\mu_{U}: A \otimes_{\mathbb{H}} U \rightarrow U$ called the module multiplication map.

(iii) The maps $\mu_{A}$ and $\mu_{U}$ define AHH-morphisms $\mu_{A} \otimes_{\mathbb{H}}$ id and id $\otimes_{\mathbb{H}} \mu_{U}$ : $A \otimes_{\mathbb{H}} A \otimes_{\mathbb{H}} U \rightarrow A \otimes_{\mathbb{H}} U$. Composing with $\mu_{U}$ gives AHI-morphisms $\mu_{U} \circ$ 
$\left(\mu_{A} \otimes_{\mathbb{H}}\right.$ id $)$ and $\mu_{U} \circ\left(\right.$ id $\left.\otimes_{\mathbb{H}} \mu_{U}\right): A \otimes_{\mathbb{H}} A \otimes_{\mathbb{H}} U \rightarrow U$. Then $\mu_{U} \circ\left(\mu_{A} \otimes_{\mathbb{H}}\right.$ id $)=$ $\mu_{U} \circ\left(\mathrm{id} \otimes_{\mathbb{H}} \mu_{U}\right)$. This is associativity of module multiplication.

(iv) For $u \in U, 1 \otimes_{\mathbb{H}} u \in A \otimes_{\mathbb{H}} U$ by Lemma 1.1.9. Then $\mu_{U}\left(1 \otimes_{\mathbb{H}} u\right)=u$ for all $u \in U$. Thus 1 acts as an identity on $U$.

Now we can define H-algebras and modules over them. Here H-algebra stands for Hamilton algebra (but my wife calls them Happy algebras).

\section{Definition 2.1.1}

- An H-algebra satisfies Axiom A.

- A noncommutative H-algebra satisfies Axiom A, except part (iii).

- Let $A$ be an H-algebra. A module $U$ over $A$ satisfies Axiom M.

An $\mathrm{H}$-algebra is basically a commutative algebra over the skew field $\mathbb{H}$. This is a strange idea: how can the algebra commute when the field does not? The obvious answer is that the algebra is only a partial algebra, and multiplication is only allowed when the elements commute. I'm not sure if this is the full story, though. In this paper our principal interest is in commutative $\mathrm{H}$-algebras, but noncommutative $\mathrm{H}$-algebras also exist.

The associative axiom $\mathrm{A}(\mathrm{iv})$ gives a good example of the issues involved in finding quaternionic analogues of algebraic structures. The usual formulation is that $(a b) c=a(b c)$ for all $a, b, c \in A$. This is not suitable for the quaternionic case, as not all elements in $A$ can be multiplied, so we rewrite the axiom in terms of linear maps of tensor products, and the quaternionic analogue becomes clear. Finally we define morphisms of H-algebras.

Definition 2.1.2 Let $A, B$ be $\mathrm{H}$-algebras, and let $\phi: A \rightarrow B$ be an AHImorphism. Write $1_{A}, 1_{B}$ for the identities in $A, B$ respectively. We say $\phi$ is a morphism of H-algebras or an H-algebra morphism if $\phi\left(1_{A}\right)=1_{B}$ and $\mu_{B} \circ\left(\phi \otimes_{\mathbb{H}} \phi\right)=\phi \circ \mu_{A}$ as AHI-morphisms $A \otimes_{\mathbb{H}} A \rightarrow B$.

\section{$2.2 \quad \mathrm{H}$-algebras and Poisson brackets}

Recall the idea of a Lie bracket on a vector space. A real algebra may be equipped with a Lie bracket satisfying certain conditions, and in this case the Lie bracket is called a Poisson bracket, and the algebra is called a Poisson algebra. Poisson algebras are studied in [7]. In this section we define one possible analogue of these concepts in our theory of quaternionic algebra. 
The analogue of a Poisson algebra will be called an HP-algebra. We begin by defining a special AHI-module.

Definition 2.2.1 Define $Y \subset \mathbb{H}^{3}$ by $Y=\left\{\left(q_{1}, q_{2}, q_{3}\right): q_{1} i_{1}+q_{2} i_{2}+q_{3} i_{3}=0\right\}$. Then $Y \cong \mathbb{H}^{2}$ is an $\mathbb{H}$-module. Define $Y^{\prime} \subset Y$ by $Y^{\prime}=\left\{\left(q_{1}, q_{2}, q_{3}\right) \in\right.$ $\left.Y: q_{j} \in \mathbb{I}\right\}$. Then $\operatorname{dim} Y^{\prime}=5$ and $\operatorname{dim} Y^{\dagger}=3$. Thus $\operatorname{dim} Y=4 j$ and $\operatorname{dim} Y^{\prime}=2 j+r$, where $j=2$ and $r=1$. Define a map $\nu: Y \rightarrow \mathbb{H}$ by $\nu\left(\left(q_{1}, q_{2}, q_{3}\right)\right)=i_{1} q_{1}+i_{2} q_{2}+i_{3} q_{3}$. Then $\operatorname{Im} \nu=\mathbb{I}$, and $\operatorname{Ker} \nu=Y^{\prime}$. But $Y / Y^{\prime} \cong\left(Y^{\dagger}\right)^{*}$, so that $\nu$ induces an isomorphism $\nu:\left(Y^{\dagger}\right)^{*} \rightarrow \mathbb{I}$. Since $\mathbb{I} \cong \mathbb{I}^{*}$, we have $\left(Y^{\dagger}\right)^{*} \cong \mathbb{I} \cong Y^{\dagger}$. It is easy to see that $Y$ is a stable AHI-module. Thus $Y \otimes_{\mathbb{H}} Y$ satisfies $\operatorname{dim}\left(Y \otimes_{\mathbb{H}} Y\right)=12$ and $\operatorname{dim}\left(Y \otimes_{\mathbb{H}} Y\right)^{\prime}=7$, by Theorem 1.2.6. Proposition 1.2.8 then shows that $S_{\mathbb{H}}^{2} Y=Y \otimes_{\mathbb{H}} Y$ and $\Lambda_{\mathbb{H}}^{2} Y=\{0\}$.

Let $A$ be an AHI-module. Here is the axiom for a Lie bracket on $A$.

Axiom P1. ( $i)$ There is an AHI-morphism $\xi_{A}: A \otimes_{\mathbb{H}} A \rightarrow A \otimes_{\mathbb{H}} Y$ called the Lie bracket or Poisson bracket, where $Y$ is the AHI-module of Definition 2.2.1.

(ii) $S_{\mathbb{H}}^{2} A \subset \operatorname{Ker} \xi_{A}$. Thus $\xi_{A}$ is antisymmetric.

(iii) There are AHI-morphisms id $\otimes_{\mathbb{H}} \xi_{A}: A \otimes_{\mathbb{H}} A \otimes_{\mathbb{H}} A \rightarrow A \otimes_{\mathbb{H}} A \otimes_{\mathbb{H}} Y$ and $\xi_{A} \otimes_{\mathbb{H}}$ id $: A \otimes_{\mathbb{H}} A \otimes_{\mathbb{H}} Y \rightarrow A \otimes_{\mathbb{H}} Y \otimes_{\mathbb{H}} Y$. Composing gives an AHI-morphism $\left(\xi_{A} \otimes_{\mathbb{H}}\right.$ id $) \circ\left(\mathrm{id} \otimes_{\mathbb{H}} \xi_{A}\right): A \otimes_{\mathbb{H}} A \otimes_{\mathbb{H}} A \rightarrow A \otimes_{\mathbb{H}} Y \otimes_{\mathbb{H}} Y$. Then $\Lambda_{\mathbb{H}}^{3} A \subset \operatorname{Ker}\left(\left(\xi_{A} \otimes_{\mathbb{H}} \mathrm{id}\right) \circ\left(\mathrm{id} \otimes_{\mathbb{H}} \xi_{A}\right)\right)$. This is the Jacobi identity for $\xi_{A}$.

For the next axiom, let $A$ be an H-algebra.

Axiom P2. ( $i)$ If $a \in A$, we have $1 \otimes_{\mathbb{H}} a \in A \otimes_{\mathbb{H}} A$. Then $\xi_{A}\left(1 \otimes_{\mathbb{H}} a\right)=0$.

(ii) There are $\mathrm{A} \mathbb{H}$-morphisms id $\otimes_{\mathbb{H}} \xi_{A}: A \otimes_{\mathbb{H}} A \otimes_{\mathbb{H}} A \rightarrow A \otimes_{\mathbb{H}} A \otimes_{\mathbb{H}} Y$ and $\mu_{A} \otimes_{\mathbb{H}}$ id $: A \otimes_{\mathbb{H}} A \otimes_{\mathbb{H}} Y \rightarrow A \otimes_{\mathbb{H}} Y$. Composing gives an AH-morphism $\left(\mu_{A} \otimes_{\mathbb{H}}\right.$ id $) \circ\left(\mathrm{id} \otimes_{\mathbb{H}} \xi_{A}\right): A \otimes_{\mathbb{H}} A \otimes_{\mathbb{H}} A \rightarrow A \otimes_{\mathbb{H}} Y$. Similarly, there are AHImorphisms $\mu_{A} \otimes_{\mathbb{H}}$ id $: A \otimes_{\mathbb{H}} A \otimes_{\mathbb{H}} A \rightarrow A \otimes_{\mathbb{H}} A$ and $\xi_{A}: A \otimes_{\mathbb{H}} A \rightarrow A \otimes_{\mathbb{H}} Y$. Composing gives an AII-morphism $\xi_{A} \circ\left(\mu_{A} \otimes_{\mathbb{H}}\right.$ id $): A \otimes_{\mathbb{H}} A \otimes_{\mathbb{H}} A \rightarrow A \otimes_{\mathbb{H}} Y$. Then $\xi_{A} \circ\left(\mu_{A} \otimes_{\mathbb{H}} \mathrm{id}\right)=2\left(\mu_{A} \otimes_{\mathbb{H}} \mathrm{id}\right) \circ\left(\mathrm{id} \otimes_{\mathbb{H}} \xi_{A}\right)$ on $S_{\mathbb{H}}^{2} A \otimes_{\mathbb{H}} A$. This is the derivation property.

Now we can define HL-algebras and HP-algebras. Here HL-algebra stands for Hamilton-Lie algebra, and HP-algebra stands for Hamilton-Poisson algebra (but my wife calls these Happy Fish algebras).

\section{Definition 2.2.2}


- an $H L$-algebra is an AHI-module $A$ satisfying Axiom P1.

- an HP-algebra satisfies Axioms A, P1 and P2.

Here is a little motivation for the definitions above. If $M$ is a symplectic manifold, then the algebra of smooth functions on $M$ acquires a Poisson bracket. Since a hyperkähler manifold $M$ has 3 symplectic structures, the algebra of smooth functions on $M$ has 3 Poisson brackets, and these interact with the $\mathrm{H}$-algebra $A$ of q-holomorphic functions on $M$, that will be defined in Chapter 3.

Our definition of HP-algebra is an attempt to capture the essential algebraic properties of this interaction. We may regard $A \otimes_{\mathbb{H}} Y$ as a subspace of $A \otimes\left(Y^{\dagger}\right)^{*}$, and $\left(Y^{\dagger}\right)^{*} \cong \mathbb{I}$ by Definition 2.2.1. Thus $\xi_{A}$ is an antisymmetric map from $A \otimes_{\mathbb{H}} A$ to $A \otimes \mathbb{I}$, i.e. a triple of antisymmetric maps from $A \otimes_{\mathbb{H}} A$ to $A$. These 3 antisymmetric maps should be interpreted as the 3 Poisson brackets on the hyperkähler manifold.

\subsection{Filtered and graded $\mathrm{H}$-algebras}

We begin by defining filtered and graded AHI-modules.

Definition 2.3.1 Let $U$ be an AHI-module. A filtration of $U$ is a sequence $U_{0}, U_{1}, \ldots$ of AHH-submodules of $U$, such that $U_{j} \subset U_{k}$ whenever $j \leq k$, and $U=\bigcup_{k=0}^{\infty} U_{k}$. We call $U$ a filtered AH-module if it has a filtration $U_{0}, U_{1}, \ldots$

Let $U$ be an AHI-module. A grading of $U$ is a sequence $U^{0}, U^{1}, \ldots$ of AHI-submodules of $U$, such that $U=\bigoplus_{k=0}^{\infty} U^{k}$. We call $U$ a graded AHImodule if it has a grading $U^{0}, U^{1}, \ldots$ If $U$ is a graded AHI-module, define $U_{k}=\bigoplus_{j=0}^{k} U^{j}$. Then $U_{0}, U_{1}, \ldots$ is a filtration of $U$, so every graded AHImodule is also a filtered AHI-module.

Let $U, V$ be filtered AHI-modules, and $\phi: U \rightarrow V$ be an AHI-morphism. We say that $\phi$ is a filtered AH-morphism if $\phi\left(U_{k}\right) \subset V_{k}$ for each $k \geq 0$. Graded AHI-morphisms are also defined in the obvious way.

Here are axioms for filtered and graded H- and HP-algebras.

Axiom AF. $(i) A$ is a filtered AHI-module.

(ii) $\mathbb{H} \cdot 1 \subset A_{0}$.

(iii) For each $j, k, \mu_{A}\left(A_{j} \otimes_{\mathbb{H}} A_{k}\right) \subset A_{j+k}$.

Axiom AG. $(i) A$ is a graded AHI-module. 
(ii) $\mathbb{H} \cdot 1 \subset A^{0}$.

(iii) For each $j, k, \mu_{A}\left(A^{j} \otimes_{\mathbb{H}} A^{k}\right) \subset A^{j+k}$.

Axiom PF. For each $j, k, \xi_{A}\left(A_{j} \otimes_{\mathbb{H}} A_{k}\right) \subset A_{j+k-1} \otimes_{\mathbb{H}} Y$.

Axiom PG. For each $j, k, \xi_{A}\left(A^{j} \otimes_{\mathbb{H}} A^{k}\right) \subset A^{j+k-1} \otimes_{\mathbb{H}} Y$.

\section{Definition 2.3.2}

- A filtered H-algebra satisfies Axioms A and AF.

- A graded H-algebra satisfies Axioms A and AG.

- a filtered HP-algebra satisfies Axioms A, AF, P1, P2, and PF.

- a graded HP-algebra satisfies Axioms A, AG, P1, P2, and PG.

Morphisms of filtered and graded H-algebras are defined in the obvious way, following Definition 2.1.2. The choice of the grading $j+k-1$ in Axioms $\mathrm{PF}$ and $\mathrm{PG}$ is not always appropriate, but depends on the situation. For some of our applications, the grading $j+k-2$ is better. Now let $U$ be an AHI-module, and $V$ an AHH-submodule of $V$. Then $U / V$ is naturally an $\mathbb{H}$ module. As $V^{\prime}=U^{\prime} \cap V$, we may interpret $U^{\prime} / V^{\prime}$ as a real vector subspace of $U / V$. Put $(U / V)^{\prime}=U^{\prime} / V^{\prime}$. Then $U / V$ is an $\mathbb{H}$-module with a real vector subspace $(U / V)^{\prime}$. Note that $U / V$ may or may not be an AHI-module, because it may not satisfy the condition of Definition 1.1.1.

Definition 2.3.3 Let $A$ be a filtered H-algebra. Define $A_{k}=\{0\}$ for $k<0$ in $\mathbb{Z}$. We say that $A$ is a stable filtered $H$-algebra, or $S F H$-algebra, if for each $k \geq 0, A_{k} / A_{k-1}$ is a stable AHI-module. Let $B$ be a graded H-algebra. We say $B$ is a stable graded H-algebra, or $S G H$-algebra, if $B^{k}$ is stable for each $k \geq 0$.

Lemma 2.3.4 Let $A$ be an SFH-algebra. Then for each $j, k \geq 0, A_{k}$ and $A_{j} / A_{j-k}$ are stable $\mathrm{AHI}-$ modules. Let $j, k \geq 0$ and $l>0$ be integers. Then the multiplication map $\mu_{A}: A_{j} \otimes_{\mathbb{H}} A_{k} \rightarrow A_{j+k}$ induces a natural AHI-morphism $\mu_{j k l}^{A}:\left(A_{j} / A_{j-l}\right) \otimes_{\mathbb{H}}\left(A_{k} / A_{k-l}\right) \rightarrow A_{j+k} / A_{j+k-l}$.

Proof. We shall prove that $A_{k}$ is stable, by induction on $k$. Firstly, $A_{0}=$ $A_{0} / A_{-1}$ is stable, by definition. Suppose by induction that $A_{k-1}$ is stable. The sequence $0 \rightarrow A_{k-1} \rightarrow A_{k} \stackrel{\pi_{k}}{\longrightarrow} A_{k} / A_{k-1} \rightarrow 0$ is AHI-exact, and $A_{k-1}$ and 
$A_{k} / A_{k-1}$ are stable. Therefore, Proposition 1.3 .5 shows that $A_{k}$ is stable, so all $A_{k}$ are stable, by induction. By a similar argument involving induction on $k, A_{j} / A_{j-k}$ is stable.

Now let $j, k, l$ be as given, and let $\pi_{m}: A_{m} \rightarrow A_{m} / A_{m-l}$ be the natural projection, for $m \geq 0$. Because $0 \rightarrow A_{j-l} \rightarrow A_{j} \stackrel{\pi_{j}}{\rightarrow} A_{j} / A_{j-l} \rightarrow 0$ and $0 \rightarrow A_{k-l} \rightarrow A_{k} \stackrel{\pi_{k}}{\longrightarrow} A_{k} / A_{k-l} \rightarrow 0$ are AHI-exact sequences of stable AHImodules, two applications of Proposition 1.3 .4 show that the sequence

$$
A_{j} \otimes_{\mathbb{H}} A_{k} \stackrel{\pi_{j} \otimes_{\mathbb{H}} \pi_{k}}{\longrightarrow}\left(A_{j} / A_{j-l}\right) \otimes_{\mathbb{H}}\left(A_{k} / A_{k-l}\right) \rightarrow 0
$$

is AHI-exact at the middle term. Now $\mu_{A}$ maps $A_{j} \otimes_{\mathbb{H}} A_{k}$ to $A_{j+k}$. By identifying the kernels of $\pi_{j} \otimes_{\mathbb{H}} \pi_{k}$ and $\pi_{j+k} \circ \mu_{A}$, it can be seen that there exists a linear map $\mu_{j k l}^{A}$ as in the lemma, such that $\mu_{j k l}^{A} \circ\left(\pi_{j} \otimes_{\mathbb{H}} \pi_{k}\right)=\pi_{j+k} \circ \mu_{A}$ as maps $A_{j} \otimes_{\mathbb{H}} A_{k} \rightarrow A_{j+k} / A_{j+k-l}$. Using the AHI-exactness of (13) and that $\pi_{j+k} \circ \mu_{A}$

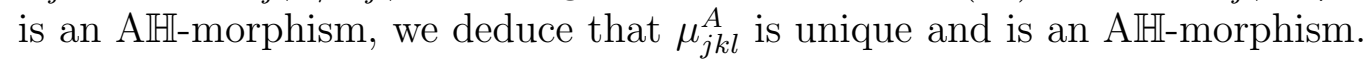

Using this lemma, we make a definition.

Definition 2.3.5 Let $A, B$ be SFH-algebras and let $l>0$ be an integer. By Lemma 2.3.4, $A_{j} / A_{j-l}$ and $B_{j} / B_{j-l}$ are stable AHI-modules for each $j \geq 0$. We say that $A$ and $B$ are isomorphic to order $l$ if the following holds. For $j \geq 0$ there are AHI-isomorphisms $\phi_{j}: A_{j} / A_{j-l} \rightarrow B_{j} / B_{j-l}$. These satisfy $\phi_{j}\left(A_{k} / A_{j-l}\right) \subset B_{k} / B_{j-l}$ when $j-l \leq k \leq j$. Therefore $\phi_{j}$ projects to a map $A_{j} / A_{j-l+1} \rightarrow B_{j} / B_{j-l+1}$, and $\phi_{j+1}$ restricts to a map $A_{j} / A_{j-l+1} \rightarrow$ $B_{j} / B_{j-l+1}$. Then $\phi_{j}=\phi_{j+1}$ on $A_{j} / A_{j-l+1}$. Also, for all $j, k \geq 0, \mu_{j k l}^{B} \circ$ $\left(\phi_{j} \otimes_{\mathbb{H}} \phi_{k}\right)=\phi_{j+k} \circ \mu_{j k l}^{A}$ as AHI-morphisms from $\left(A_{j} / A_{j-l}\right) \otimes_{\mathbb{H}}\left(A_{k} / A_{k-l}\right)$ to $B_{j+k} / B_{j+k-l}$. Here $\mu_{j k l}^{A}$ and $\mu_{j k l}^{B}$ are defined in Lemma 2.3.4.

There is a well-known way to construct a graded algebra from a filtered algebra (for instance [7, p. 35-37] gives the associated graded Poisson algebra of a filtered Poisson algebra). Here is the analogue of this for $\mathrm{H}$-algebras, which will be applied in $\S 3.5$.

Proposition 2.3.6 Let $A$ be an SFH-algebra. Define $B^{k}=A_{k} / A_{k-1}$ for $k \geq 0$. Define $B=\bigoplus_{k=0}^{\infty} B^{k}$. Then $B$ has the structure of an SGH-algebra, in a natural way.

Proof. As $A$ is an SFH-algebra, $B^{k}$ is a stable A $\mathbb{H}$-module, by definition. Let $j, k \geq 0$ be integers. Putting $l=1$, Lemma 2.3.4 defines an AHI-morphism 
$\mu_{j k 1}^{A}: B^{j} \otimes_{\mathbb{H}} B^{k} \rightarrow B^{j+k}$. Let $\mu_{B}: B \otimes_{\mathbb{H}} B \rightarrow B$ be the unique AHI-morphism, such that the restriction of $\mu_{B}$ to $B^{j} \otimes_{\mathbb{H}} B^{k}$ is $\mu_{j k 1}^{A}$. It is elementary to show that because $A$ is an $\mathrm{H}$-algebra, $\mu_{B}$ makes $B$ into an H-algebra, and we leave this to the reader. As $B$ satisfies Axiom AG, $B$ is graded, so $B$ is an $\mathrm{SGH}-$ algebra, and the proposition is complete.

We call the SGH-algebra $B$ defined in Proposition 2.3.6 the associated graded $H$-algebra of $A$. Note that $A$ is isomorphic to $B$ to order 1 , in the sense of Lemma 2.3.5. One might ask if the construction would work even if $A$ were only a filtered $\mathrm{H}$-algebra. There are two problems here: firstly, $B^{k}$ might not be an AHH-module, and secondly, even if $B^{j}$ and $B^{k}$ were AHImodules, the map $\pi_{j} \otimes_{\mathbb{H}} \pi_{k}: A_{j} \otimes_{\mathbb{H}} A_{k} \rightarrow B^{j} \otimes_{\mathbb{H}} B^{k}$ might not be surjective. If it were not, we could only define $\mu_{j k 1}^{A}$ uniquely on part of $B^{j} \otimes_{\mathbb{H}} B^{k}$. For these reasons we prefer $\mathrm{SFH}$-algebras.

\subsection{Free and finitely-generated $\mathrm{H}$-algebras}

First we define ideals in SFH-algebras.

Definition 2.4.1 Let $A$ be an SFH-algebra, and $I$ an AH-submodule of $A$. Set $I_{k}=I \cap A_{k}$ for $k \geq 0$. We say that $I$ is a stable filtered ideal in $A$ if $1 \notin I$, $\mu_{A}\left(I \otimes_{\mathbb{H}} A\right) \subset I$, and for each $k \geq 0, I_{k}$ and $A_{k} / I_{k}$ are stable AHH-modules.

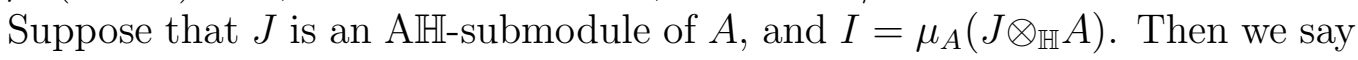
that $I$ is generated by $J$.

The proof of the next lemma is similar to that of Proposition 2.3.6, so we omit it.

Lemma 2.4.2 Let $A$ be an SFH-algebra, and I a stable filtered ideal in A. Then there exists a unique $S F H$-algebra $B$, with a filtered H-algebra morphism

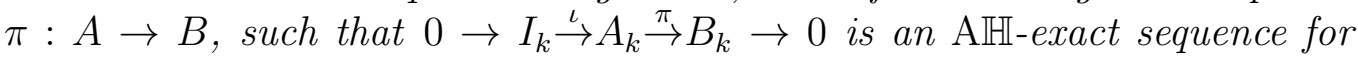
each $k \geq 0$. Here $\iota: I \rightarrow A$ is the inclusion map.

We shall call this new $\mathrm{H}$-algebra $B$ the quotient of $A$ by $I$. Next, here is the definition of a free $\mathrm{H}$-algebra.

Definition 2.4.3 Let $Q$ be an AH-module. Define the free H-algebra $F^{Q}$ generated by $Q$ as follows. Put $F^{Q}=\bigoplus_{k=0}^{\infty} S_{\mathbb{H}}^{k} Q$. Then $F^{Q}$ is an AHH-module. Now $\left(S_{\mathbb{H}}^{k} Q\right) \otimes_{\mathbb{H}}\left(S_{\mathbb{H}}^{l} Q\right) \subset \bigotimes_{\mathbb{H}}^{k+l} Q$, and from Definition 1.1.6 there is an A $\mathbb{H}-$ module projection $\sigma_{\mathbb{H}}: \bigotimes_{\mathbb{H}}^{k+l} Q \rightarrow S_{\mathbb{H}}^{k+l} Q$. Define $\mu_{k, l}:\left(S_{\mathbb{H}}^{k} Q\right) \otimes_{\mathbb{H}}\left(S_{\mathbb{H}}^{l} Q\right) \rightarrow$ $S_{\mathbb{H}}^{k+l} Q$ to be the restriction of $\sigma_{\mathbb{H}}$ to $\left(S_{\mathbb{H}}^{k} Q\right) \otimes_{\mathbb{H}}\left(S_{\mathbb{H}}^{l} Q\right)$. 
Define $\mu_{F^{Q}}: F^{Q} \otimes_{\mathbb{H}} F^{Q} \rightarrow F^{Q}$ to be the unique linear map such that the restriction of $\mu_{F} Q$ to $\left(S_{\mathbb{H}}^{k} Q\right) \otimes_{\mathbb{H}}\left(S_{\mathbb{H}}^{l} Q\right)$ is $\mu_{k, l}$. Recall that $S_{\mathbb{H}}^{0} Q=\mathbb{H}$, and define $1 \in F^{Q}$ to be $1 \in \mathbb{H}=S_{\mathbb{H}}^{0} Q$. It is easy to show that with these definitions, $F^{Q}$ is an H-algebra. The natural grading on $F^{Q}$ is $\left(F^{Q}\right)^{k}=S_{\mathbb{H}}^{k} Q$ for $k \geq 0$. The natural filtration on $F^{Q}$ is $F_{k}^{Q}=\bigoplus_{j=0}^{k}\left(F^{Q}\right)^{j}$ for $k \geq 0$.

If $Q$ is stable, then $S_{\mathbb{H}}^{k} Q$ is stable by Proposition 1.2.8. Thus, if $Q$ is stable then $F^{Q}$ is an $\mathrm{SFH}$-algebra (SGH-algebra) with the natural filtration (grading). The proof of the next lemma is trivial, and we omit it.

Lemma 2.4.4 Let $A$ be an H-algebra, and let $Q \subset A$ be an AHI-submodule. Let $\iota_{Q}: Q \rightarrow F^{Q}$ be the inclusion map. Then there is a unique H-algebra morphism $\phi_{Q}: F^{Q} \rightarrow A$ such that $\phi_{Q} \circ \iota_{Q}: Q \rightarrow A$ is the identity on $Q$.

Definition 2.4.5 A free SFH-algebra is an SFH-algebra $A$ that is isomorphic, as an H-algebra, to some $F^{Q}$, for finite-dimensional $Q$. Note that the filtration on $A$ need not be the natural filtration on $F^{Q}$. A finitely-generated $S F H$-algebra, or FGH-algebra is the quotient $B$ of a free SFH-algebra $A$ by a stable filtered ideal $I$ in $A$. Suppose that $Q$ is an AHI-submodule of $B$, and that there exists an H-algebra isomorphism $A \cong F^{Q}$ identifying the maps $\pi: A \rightarrow B$ and $\phi_{Q}: F^{Q} \rightarrow B$. Then we say that $Q$ generates the FGH-algebra $B$.

The purpose of this definition is as follows. The polynomials on an affine algebraic variety form a finitely-generated, filtered algebra, and in algebraic geometry one studies this algebra to learn about the variety. In the opinion of the author, FGH-algebras are the best quaternionic analogue of finitelygenerated, filtered algebras. Clearly, they are finitely-generated, filtered Halgebras, and the extra stability conditions we impose enable us to exploit the 'right-exactness' results of $\$ 1.3$.

Moreover, the author believes that there is a wide class of noncompact hypercomplex manifolds, to which one can naturally associate an FGH-algebra. The study of FGH-algebras should be interpreted as the 'quaternionic algebraic geometry' of these hypercomplex manifolds. Therefore, the author proposes that the study of FGH-algebras, from the algebraic point of view, may be interesting and worthwhile. More will be said on these ideas in Chapter 4 .

We leave the proof of this final result as an exercise. It will be useful later. 
Proposition 2.4.6 Let $A$ be an SFH-algebra, and $B$ the associated graded algebra, as in $\$ 2.3$. Then $B$ is also an SFH-algebra. Suppose that $B$ is an $F G H$-algebra, generated by $B_{k}$. Then $A$ is an FGH-algebra, generated by $A_{k}$.

\section{$3 \quad$ Hypercomplex geometry}

We begin in 3.1 by defining hypercomplex manifolds, hyperkähler manifolds and q-holomorphic functions on hypercomplex manifolds, and some elementary properties of q-holomorphic functions are given. Section 3.2 proves that the vector space $A$ of q-holomorphic functions on a hypercomplex manifold $M$ forms an $\mathrm{H}$-algebra, and $\S 3.3$ shows that if $M$ is hyperkähler, then $A$ is an HP-algebra. Section 3.4 discusses the possibility of reconstructing a hypercomplex manifold from an $\mathrm{H}$-algebra of q-holomorphic functions upon it. Finally, 8.5 discusses hyperkähler manifolds that are asymptotic to a conical metric.

\subsection{Q-holomorphic functions on hypercomplex mani- folds}

We begin by defining hypercomplex manifolds ([26, p. 137-139]) and hyperkähler manifolds ([26, p. 114-123]). Let $M$ be a manifold of dimension $4 n$. A hypercomplex structure on $M$ is a triple $\left(I_{1}, I_{2}, I_{3}\right)$ on $M$, where $I_{j}$ is a complex structure on $M$, and $I_{1} I_{2}=I_{3}$. A hyperkähler structure on $M$ is a quadruple $\left(g, I_{1}, I_{2}, I_{3}\right)$, where $g$ is a Riemannian metric on $M,\left(I_{1}, I_{2}, I_{3}\right)$ is a hypercomplex structure on $M$, and $g$ is Kähler w.r.t. each $I_{j}$. If $M$ has a hypercomplex (hyperkähler) structure, then $M$ is called a hypercomplex (hyperkähler) manifold.

If $M$ is a hypercomplex manifold, then $I_{1}, I_{2}, I_{3}$ satisfy the quaternion relations, so that each tangent space $T_{m} M$ is an $\mathbb{H}$-module isomorphic to $\mathbb{H}^{n}$. Also, if $r_{1}, r_{2}, r_{3} \in \mathbb{R}$ with $r_{1}^{2}+r_{2}^{2}+r_{3}^{2}=1$, then $r_{1} I_{1}+r_{2} I_{2}+r_{3} I_{3}$ is a complex structure. Thus a hypercomplex manifold possesses a 2-dimensional family of integrable complex structures, parametrized by $\mathcal{S}^{2}$. We will often use $\mathcal{S}^{2}$ to denote this family of complex structures.

Let $M$ be a hypercomplex manifold. For $k \geq 0$, define $\Omega^{k}=C^{\infty}\left(\Lambda^{k} T^{*} M\right)$, and $\Omega^{k}(\mathbb{H})=C^{\infty}\left(\mathbb{H} \otimes \Lambda^{k} T^{*} M\right)$. Then $\Omega^{1}$ is the vector space of smooth 1forms on $M$, and $\Omega^{0}(\mathbb{H})$ is the vector space of smooth, quaternion-valued 
functions on $M$. Define an operator $D: \Omega^{0}(\mathbb{H}) \rightarrow \Omega^{1}$ by

$$
D\left(a_{0}+a_{1} i_{1}+a_{2} i_{2}+a_{3} i_{3}\right)=d a_{0}+I_{1}\left(d a_{1}\right)+I_{2}\left(d a_{2}\right)+I_{3}\left(d a_{3}\right),
$$

where $a_{0}, \ldots, a_{3}$ are smooth real functions on $M$.

We define a $q$-holomorphic function on $M$ to be an element $a=a_{0}+a_{1} i_{1}+$ $a_{2} i_{2}+a_{3} i_{3}$ of $\Omega^{0}(\mathbb{H})$ for which $D(a)=0$. The term q-holomorphic is short for quaternion-holomorphic, and it is intended to indicate that a q-holomorphic function on a hypercomplex manifold is the appropriate quaternionic analogue of a holomorphic function on a complex manifold. The operator $D$ of (14) should be thought of as the quaternionic analogue of the $\bar{\partial}$ operator on a complex manifold. It can be seen that $D(a)=0$ is equivalent to the equation

$$
d a-I_{1}(d a) i_{1}-I_{2}(d a) i_{2}-I_{3}(d a) i_{3}=0,
$$

where each term is an $\mathbb{H}$-valued 1 -form, $I_{j}$ acts on 1 -forms and $i_{j}$ acts on $\mathbb{H}$ by multiplication.

Now in 1935, Fueter defined a class of 'regular' $\mathbb{H}$-valued functions on $\mathbb{H}$, using an analogue of the Cauchy-Riemann equations, and Fueter and his coworkers went on to develop the theory of quaternionic analysis, by analogy with complex analysis. An account of this theory, with references, is given by Sudbery in [27]. On the hypercomplex manifold $\mathbb{H}$, Fueter's definition of regular function coincides with that of q-holomorphic function, given above. We shall make little reference to Fueter's theory, because we are interested in rather different questions. However, in Chapter 4 we will use our theory to give an elegant construction of the spaces of homogeneous q-holomorphic functions on $\mathbb{H}$, which are important in quaternionic analysis.

Suppose that $M$ is hyperkähler. Then using the metric $g$ on $M$ we construct the operator $D^{*}: \Omega^{1} \rightarrow \Omega^{0}(\mathbb{H})$, which is given by

$$
D^{*}(\alpha)=d^{*} \alpha-d^{*}\left(I_{1} \alpha\right) i_{1}-d^{*}\left(I_{2} \alpha\right) i_{2}-d^{*}\left(I_{3} \alpha\right) i_{3} .
$$

Now for a smooth real function $f$ on a Kähler manifold, $d^{*}(I d f)=0$. Using this we can show that $D^{*} D(a)=\Delta a$, where $\Delta$ is the usual Laplacian. Thus q-holomorphic functions on a hyperkähler manifold are harmonic. When $n=1, D$ is elliptic, and is the Dirac operator $D_{+}$. When $n>1, D$ is overdetermined elliptic.

Here are two basic properties of q-holomorphic functions. 
Lemma 3.1.1 Suppose that a is $q$-holomorphic on $M$, and that $q \in \mathbb{H}$. Then qa is q-holomorphic.

Suppose that $i=r_{1} i_{1}+r_{2} i_{2}+r_{3} i_{3} \in \mathbb{I}$ satisfies $i^{2}=-1$, and that $I=$ $r_{1} I_{1}+r_{2} I_{2}+r_{3} I_{3}$ is the corresponding complex structure on $M$. Suppose that $y+z i$ is a complex function on $M$ that is holomorphic w.r.t. I. Then $y+z i$ is q-holomorphic on $M$, regarding $y+z i=y+z r_{1} i_{1}+z r_{2} i_{2}+z r_{3} i_{3}$ as an element of $\Omega^{0}(\mathbb{H})$.

Proof. Let $q=q_{0}+q_{1} i_{1}+q_{2} i_{2}+q_{3} i_{3} \in \mathbb{H}$, and define $Q=q_{0}+q_{1} I_{1}+q_{2} I_{2}+q_{3} I_{3}$, regarding $Q$ as an endomorphism of $T^{*} M$. Let $a \in \Omega^{0}(\mathbb{H})$. It is easy to verify that $D(q a)=Q \cdot D(a)$. Thus $D(q a)=0$ if $D(a)=0$, and $q a$ is q-holomorphic whenever $a$ is q-holomorphic. This proves the first part.

If $y+z i$ is holomorphic w.r.t. $I$, then $d y+I(d z)=0$ by the CauchyRiemann equations. But

$$
\begin{aligned}
0 & =d y+I(d z)=d y+\left(r_{1} I_{1}+r_{2} I_{2}+r_{3} I_{3}\right)(d z) \\
& =D\left(y+z r_{1} i_{1}+z r_{2} i_{2}+z r_{3} i_{3}\right),
\end{aligned}
$$

so that $y+z r_{1} i_{1}+z r_{2} i_{2}+z r_{3} i_{3}$ is q-holomorphic. This completes the lemma.

The operator $D$ of (14) was also studied by Baston [5], upon quaternionic manifolds rather than hypercomplex manifolds. He calls $D$ the Dirac-Fueter operator, and uses the Penrose transform to interpret $D$ as a holomorphic object on the twistor space $Z$ of $M$. Baston [5, p. 44-45] shows that Ker $D$ on a quaternionic manifold $M$ can be identified with the sheaf cohomology group $H^{1}\left(Z, \mathcal{O}_{Z}(-3)\right)$, giving a twistor interpretation of q-holomorphic functions. He also constructs an exact complex of operators resolving $D$, [5, p. 43-44].

\subsection{Hypercomplex manifolds and $\mathrm{H}$-algebras}

Let $M$ be a hypercomplex manifold, and let $A$ be the vector space of qholomorphic functions on $M$. In this section we will prove that $A$ is an H-algebra.

Definition 3.2.1 Let $M$ be a hypercomplex manifold. Define $A \subset \Omega^{0}(\mathbb{H})$ to be the vector space of q-holomorphic functions on $M$. Let $a \in A$, and define $(q \cdot a)(m)=q(a(m))$ for $m \in M$. Then $q \cdot a \in A$ by Lemma 3.1.1, and this gives an $\mathbb{H}$-action on $A$, so $A$ is an $\mathbb{H}$-module. Define a subspace $A^{\prime}$ 
in $A$ by $A^{\prime}=\{a \in A: a(m) \in \mathbb{I}$ for all $m \in M\}$. For each $m \in M$, define $\theta_{m}: A \rightarrow \mathbb{H}$ by $\theta_{m}(a)=a(m)$. Then $\theta_{m} \in A^{\times}$, and if $a \in A^{\prime}$ then $\theta_{m}(a) \in \mathbb{I}$, so that $\theta_{m} \in A^{\dagger}$.

Suppose $a \in A$, and $\alpha(a)=0$ for all $\alpha \in A^{\dagger}$. Since $\theta_{m} \in A^{\dagger}, a(m)=0$ for each $m \in M$, and so $a=0$. Thus $A$ is an AHI-module, by Definition 1.1.1. Define the element $1 \in A$ to be the constant function on $M$ with value 1 . Then $1 \notin A^{\prime}$, but $\mathbb{I} \cdot 1 \subset A^{\prime}$. We will also write $A_{M}$ for $A$, when we wish to specify the manifold $M$.

The following proposition gives us a greater understanding of the quaternionic tensor product.

Proposition 3.2.2 Let $M$ and $N$ be hypercomplex manifolds, and let $U, V$ be $\mathrm{AH}$-submodules of the $\mathrm{AH}$-modules $A_{M}, A_{N}$ of q-holomorphic functions on $M, N$ respectively. Define $W$ to be the vector space of smooth, $\mathbb{H}$-valued functions $w$ on $M \times N$, such that for each $m \in M$, the function $n \mapsto w(m, n)$ lies in $V$, and for each $n \in N$, the function $m \mapsto w(m, n)$ lies in $U$. Then each such $w$ is a q-holomorphic function on $M \times N$, and $W$ is an $\mathrm{AH}$ submodule of $A_{M \times N}$. Also, there is a canonical injective AHI-morphism $\phi$ : $U \otimes_{\mathbb{H}} V \rightarrow W$. If $U, V$ are finite-dimensional, $\phi$ is an $\mathrm{AH}-i$ somorphism.

Proof. Suppose $w: M \times N \rightarrow \mathbb{H}$ is a smooth function, such that for each $m \in M$, the function $n \mapsto w(m, n)$ lies in $V$, and for each $n \in N$, the function $m \mapsto w(m, n)$ lies in $U$. The condition for $w$ to be q-holomorphic is $D(w)=0$. But $D(w)=D_{M}(w)+D_{N}(w)$, where $D_{M}$ involves only derivatives in the $M$ directions, and $D_{N}$ only derivatives in the $N$ directions.

Let $n \in N$. Then the function $m \mapsto w(m, n)$ is equal to some $u \in U$. Thus $D_{M}(w)(m, n)=D(u)(m)$. But the functions in $U$ are q-holomorphic, so $D(u)=0$. Therefore $D_{M}(w)=0$, and similarly $D_{N}(w)=0$. So $D(w)=0$, and $w$ is q-holomorphic, as we have to prove. It is clear that the space $W$ of such functions $w$ is closed under addition and multiplication by $\mathbb{H}$. Thus $W$ is an $\mathbb{H}$-submodule of $A_{M \times N}$, so $W$ is an AH-submodule of $A_{M \times N}$.

Now let $\epsilon \in U \otimes_{\mathbb{H}} V$. Then $\epsilon \in \mathbb{H} \otimes\left(U^{\dagger}\right)^{*} \otimes\left(V^{\dagger}\right)^{*}$, so $\epsilon$ defines a linear map $\epsilon: U^{\dagger} \otimes V^{\dagger} \rightarrow \mathbb{H}$. Define a map $w: M \times N \rightarrow \mathbb{H}$ by $w(m, n)=\epsilon\left(\theta_{m} \otimes \theta_{n}\right)$. For $m \in M$, define $w_{m}: N \rightarrow \mathbb{H}$ by $w_{m}(n)=w(m, n)$. Since $\epsilon \in U \otimes_{\mathbb{H}} V$, $\epsilon \in\left(U^{\dagger}\right)^{*} \otimes \iota_{V}(V)$, so $w_{m} \in \iota(V)$, regarding $w_{m}$ as an element of $\mathbb{H} \otimes\left(V^{\dagger}\right)^{*}$. Thus $w_{m} \in V$. Similarly, defining $w_{n}(m)=w(m, n)$ for $n \in N$, we find $w_{n} \in U$ for each $n \in N$. 
To show that $w \in W$, we only need to show that $w$ is smooth. In general, if $f$ is a function on $M \times N$, such that for each $m \in M$, the function $n \mapsto f(m, n)$ is smooth, and for each $n \in N$, the function $m \mapsto f(m, n)$ is smooth, it does not follow that $f$ is smooth. However, because real tensor products involve only finite sums as in $\S 1.1, \epsilon \in\left(U^{\dagger}\right)^{*} \otimes \iota_{V}(V)$ implies that $w_{m}$ lies in some finite-dimensional subspace of $V$ for all $m \in M$, and similarly $w_{n}$ lies in a finite-dimensional subspace of $U$ for all $n \in N$. These imply that $w$ is smooth. Thus $w \in W$.

Define $\phi(\epsilon)=w$. In this way we define a map $\phi: U \otimes_{\mathbb{H}} V \rightarrow W$. It is

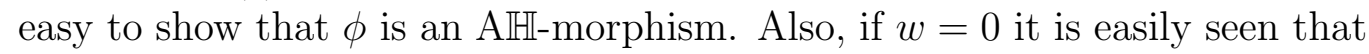
$\epsilon=0$, so $\phi$ is injective. Thus $\phi$ is an injective AHH-morphism, as we have to prove. Suppose $U, V$ are finite-dimensional, and let $w \in W$. We must find $\epsilon$ in $U \otimes_{\mathbb{H}} V$ such that $\phi(\epsilon)=w$.

Choose bases of the form $\left\{\theta_{m_{b}}: b=1, \ldots, k\right\}$ for $U^{\dagger}$ and $\left\{\theta_{n_{c}}: c=\right.$ $1, \ldots, l\}$ for $V^{\dagger}$. It can be shown that such bases exist. Let $\epsilon: U^{\dagger} \otimes V^{\dagger} \rightarrow \mathbb{H}$ be the unique linear map satisfying $\epsilon\left(\theta_{m_{b}} \otimes \theta_{n_{c}}\right)=w\left(m_{b}, n_{c}\right)$ for $b=1, \ldots, k$, $c=1, \ldots, l$. One may prove that $\epsilon \in U \otimes_{\mathbb{H}} V$, and $\phi(\epsilon)=w$. Thus $\phi$ is an injective and surjective AHI-morphism, and clearly is an AH-isomorphism. This completes the proof.

The following lemma is trivial, and the proof will be omitted.

Lemma 3.2.3 Suppose $M$ is a hypercomplex manifold, and $N$ is a hypercomplex submanifold of $M$. If $a$ is a $q$-holomorphic function on $M$, then a $\left.\right|_{N}$ is q-holomorphic on $N$. Let $\rho: A_{M} \rightarrow A_{N}$ be the restriction map. Then $\rho$ is an AHI-morphism.

Now we can define the multiplication map $\mu_{A}$ on $A$.

Definition 3.2.4 Let $M$ be a hypercomplex manifold, and $A$ the AHmodule of q-holomorphic functions on $M$. By Proposition 3.2.2 there is a canonical AIH-morphism $\phi: A \otimes_{\mathbb{H}} A \rightarrow A_{M \times M}$. Now $M$ is embedded in $M \times M$ as the diagonal submanifold $\{(m, m): m \in M\}$, and this is a hypercomplex submanifold of $M \times M$, isomorphic to $M$ as a hypercomplex manifold. Therefore Lemma 3.2 .3 gives an AIH-morphism $\rho: A_{M \times M} \rightarrow A$. Define an AHI-morphism $\mu_{A}: A \otimes_{\mathbb{H}} A \rightarrow A$ by $\mu_{A}=\rho \circ \phi$.

Here is the main result of this section.

Theorem 3.2.5 Let $M$ be a hypercomplex manifold. Then Definition 3.2.1 defines an $\mathrm{AH}-$-module $A$ and an element $1 \in A$, and Definition 3.2.4 defines 
an $\mathrm{A} \mathbb{H}$-morphism $\mu_{A}: A \otimes_{\mathbb{H}} A \rightarrow A$. With these definitions, $A$ is an H-algebra in the sense of $\$ 2.1$.

Proof. We must show that Axiom A is satisfied. Parts ( $i)$ and $(i i)$ are trivial. For part (iii), observe that the permutation map $A \otimes_{\mathbb{H}} A \rightarrow A \otimes_{\mathbb{H}} A$ that swaps round the factors, is induced by the map $M \times M \rightarrow M \times M$ given by $\left(m_{1}, m_{2}\right) \mapsto\left(m_{2}, m_{1}\right)$. Since the diagonal submanifold is invariant under this, it follows that $\mu_{A}$ is invariant under permutation, and so $\Lambda_{\mathbb{H}}^{2} A \subset \operatorname{Ker} \mu_{A}$.

Let $\Delta_{M}^{2}$ be the 'diagonal' submanifold in $M \times M$, and let $\Delta_{M}^{3}$ be the 'diagonal' submanifold in $M \times M \times M$. We interpret part ( $i v)$ as follows. $A \otimes_{\mathbb{H}} A \otimes_{\mathbb{H}} A$ is a space of q-holomorphic functions on $M \times M \times M$. The maps $\mu_{A} \otimes_{\mathbb{H}}$ id and id $\otimes_{\mathbb{H}} \mu_{A}$ are the maps restricting to $\Delta_{M}^{2} \times M$ and $M \times \Delta_{M}^{2}$ respectively. Thus $\mu_{A} \circ\left(\mu_{A} \otimes_{\mathbb{H}} \mathrm{id}\right)$ is the result of first restricting to $\Delta_{M}^{2} \times M$ and then to $\Delta_{M}^{3}$, and $\mu_{A} \circ\left(\mathrm{id} \otimes_{\mathbb{H}} \mu_{A}\right)$ is the result of first restricting to $M \times \Delta_{M}^{2}$ and then to $\Delta_{M}^{3}$. Clearly $\mu_{A} \circ\left(\mu_{A} \otimes_{\mathbb{H}}\right.$ id $)=\mu_{A} \circ\left(\mathrm{id} \otimes_{\mathbb{H}} \mu_{A}\right)$, proving part $(i v)$.

Interestingly, the proof of part $(i v)$ does not use the associativity of quaternion multiplication. This raises the possibility of generalizing the definition to give 'associative algebras over a nonassociative field'. Part $(v)$ is given in Definition 3.2.1. Finally, part $(v i)$ follows easily from the fact that 1 is the identity in $\mathbb{H}$. Thus all of Axiom $\mathrm{A}$ of $\$ 2.1$ applies, and $A$ is an H-algebra.

One problem with the H-algebra of all q-holomorphic functions on a hypercomplex manifold is that it is too large to work with - it is not in general finitely-generated, for instance. Therefore, it is convenient to restrict to $\mathrm{H}$ subalgebras of functions satisfying some condition. The condition we shall use is that of polynomial growth.

Definition 3.2.6 Let $M$ be a hypercomplex manifold, and let $r: M \rightarrow$ $[0, \infty)$ be a given continuous function. Suppose $f \in \Omega^{0}(\mathbb{H})$ on $M$, and let $k \geq 0$ be an integer. We say that $f$ has polynomial growth of order $k$, written $f=O\left(r^{k}\right)$, if there exist positive constants $C_{1}, C_{2}$ such that $|f| \leq C_{1}+C_{2} r^{k}$ on $M$.

The H-algebra of q-holomorphic functions on $M$ is $A$. For integers $k \geq 0$, define $P_{k}=\left\{a \in A: a=O\left(r^{k}\right)\right\}$, and define $P=\bigcup_{k=0}^{\infty} P_{k}$. Then $P$ is a filtered AH-module. It is easy to see that $P$ is an $\mathrm{H}$-subalgebra of $A$, and satisfies Axiom AF of $\$ 2.3$. Thus, $P$ is a filtered H-algebra. We call $P$ the filtered H-algebra of $q$-holomorphic functions of polynomial growth on $M$, and we write $P_{M}$ for $P$ when we wish to specify the manifold $M$. 
The main example we have in mind in making this definition, is the case that $M$ is a complete, noncompact hyperkähler manifold, and $r: M \rightarrow[0, \infty)$ is the distance function from some point $m_{0} \in M$. Then $P_{k}$ is independent of the choice of base point $m_{0}$. In good cases, such as those discussed in Chapter 4, $P$ is an FGH-algebra. Again, in a good case, $P$ determines the hypercomplex structure of $M$ explicitly and uniquely. Thus we can define the hypercomplex structure of $M$ completely using only a finite-dimensional amount of algebraic data.

\subsection{Hyperkähler manifolds and HP-algebras}

Let $M$ be a hyperkähler manifold, and $A$ the vector space of q-holomorphic functions on $M$. Since $M$ is hypercomplex, $A$ is an $\mathrm{H}$-algebra by Theorem 3.2.5. In this section we will see that $A$ is also an HP-algebra in the sense of \$2.2. To save space, and because we have wandered from the main subject of the paper, we shall omit the proofs of Proposition 3.3 .2 and Theorem 3.3.4. The proofs are elementary calculations, though not especially easy, and the author can supply them to the interested reader on request.

Definition 3.3.1 Let $M$ be a hyperkähler manifold. Then $M \times M$ is also a hyperkähler manifold. Let $\Delta_{M}^{2}=\{(m, m): m \in M\}$. Then $\Delta_{M}^{2}$ is a hyperkähler submanifold of $M \times M$. We shall write $M \times M=M^{1} \times M^{2}$, using the superscripts ${ }^{1}$ and ${ }^{2}$ to distinguish the two factors. Let $\nabla$ be the Levi-Civita connection on $M$. Define $\nabla^{1}, \nabla^{2}$ to be the lift of $\nabla$ to the first and second factors of $M$ in $M \times M$ respectively. Then $\nabla^{1}$ and $\nabla^{2}$ commute. Let $\nabla^{12}$ be the Levi-Civita connection on $M \times M$. Then $\nabla^{12}=\nabla^{1}+\nabla^{2}$.

Let $x \in A_{M \times M}$. Then $\nabla^{1} \nabla^{2} x \in C^{\infty}\left(\mathbb{H} \otimes T^{*} M^{1} \otimes T^{*} M^{2}\right)$ over $M \times M$. Restrict $\nabla^{1} \nabla^{2} x$ to $\Delta_{M}^{2}$. Then $\Delta_{M}^{2} \cong M$ and $\left.\left.T^{*} M^{1}\right|_{\Delta_{M}^{2}} \cong T^{*} M^{2}\right|_{\Delta_{M}^{2}} \cong T^{*} M$. Thus $\left.\nabla^{1} \nabla^{2} x\right|_{\Delta_{M}^{2}} \in C^{\infty}\left(\mathbb{H} \otimes T^{*} M \otimes T^{*} M\right)$ over $M$. Define a linear map $\Theta: A_{M \times M} \rightarrow \Omega^{0}(\mathbb{H}) \otimes \mathbb{I}$ by

$$
\begin{aligned}
\Theta(x)=\left\{\left.g^{a b}\left(I_{1}\right)_{a}^{c} \nabla_{b}^{1} \nabla_{c}^{2} x\right|_{\Delta_{M}^{2}}\right\} \otimes i_{1} & +\left\{\left.g^{a b}\left(I_{2}\right)_{a}^{c} \nabla_{b}^{1} \nabla_{c}^{2} x\right|_{\Delta_{M}^{2}}\right\} \otimes i_{2} \\
+ & \left\{\left.g^{a b}\left(I_{3}\right)_{a}^{c} \nabla_{b}^{1} \nabla_{c}^{2} x\right|_{\Delta_{M}^{2}}\right\} \otimes i_{3},
\end{aligned}
$$

using index notation for tensors on $M$ in the obvious way. Here $g$ is the hyperkähler metric on $M$, and $I_{1}, I_{2}, I_{3}$ the complex structures.

Here are some properties of $\Theta$. 
Proposition 3.3.2 This map satisfies $\Theta(x) \in A \otimes \mathbb{I}$. Also, $\Theta: A_{M \times M} \rightarrow$ $A \otimes \mathbb{I}$ is an AHI-morphism, and if $\Theta(x)=x_{1} \otimes i_{1}+x_{2} \otimes i_{2}+x_{3} \otimes i_{3}$ and $m \in M$, then $x_{1}(m) i_{1}+x_{2}(m) i_{2}+x_{3}(m) i_{3}=0 \in \mathbb{H}$.

Now we can define the map $\xi_{A}$ of $\$ 2.2$.

Definition 3.3.3 Proposition 3.2.2 defines an AHI-morphism $\phi: A \otimes_{\mathbb{H}} A \rightarrow$ $A_{M \times M}$. Definition 3.3.1 and Proposition 3.3.2 define an AHI-morphism $\Theta$ : $A_{M \times M} \rightarrow A \otimes \mathbb{I}$. Let $Y$ be the AHI-module of Definition 2.2.1. Then $\left(Y^{\dagger}\right)^{*} \cong \mathbb{I}$. Recall that $A \cong \iota_{A}(A)$, so we may identify $A \otimes \mathbb{I} \cong \iota_{A}(A) \otimes\left(Y^{\dagger}\right)^{*} \subset \mathbb{H} \otimes\left(A^{\dagger}\right)^{*} \otimes$ $\left(Y^{\dagger}\right)^{*}$. Define $\xi_{A}: A \otimes_{\mathbb{H}} A \rightarrow \iota_{A}(A) \otimes\left(Y^{\dagger}\right)^{*}$ to be the composition $\xi_{A}=\Theta \circ \phi$.

Here is the main result of this section.

Theorem 3.3.4 This $\xi_{A}$ maps $A \otimes_{\mathbb{H}} A$ to $A \otimes_{\mathbb{H}} Y$. It is an AH-morphism, and satisfies Axioms P1 and P2 of $\$$ 2.9. Thus, by Theorem 3.2.5 and Definition 2.2.9, if $M$ is a hyperkähler manifold, then the vector space $A$ of q-holomorphic functions on $M$ is an HP-algebra.

Given a continuous function $r: M \rightarrow[0, \infty)$, Definition 3.2.6 defines the filtered H-algebra $P$ of q-holomorphic functions on $M$ of polynomial growth. It is natural to ask whether $P$ is a filtered HP-algebra. One must show that Axiom PF of $\$ 2.3$ holds, which relates the Poisson bracket and the filtration. This is not automatic, but depends on the asymptotic properties of the hyperkähler structure and the function $r$ on $M$, and must be verified for each case. These properties also determine whether $P$ is closed under $\xi_{A}$ at all.

\subsection{Reconstructing a hypercomplex manifold from its H-algebra}

In $\$ 3.2$ we saw that the vector space of q-holomorphic functions on a hypercomplex manifold is an $\mathrm{H}$-algebra, providing a transform from geometric to algebraic objects. Now we shall consider whether this transform can be reversed. It turns out that under certain circumstances, an H-algebra does explicitly determine a unique hypercomplex manifold. Therefore, it should be possible to construct new hypercomplex manifolds by writing down their $\mathrm{H}$-algebras. 
Throughout this section, let $M$ be a hypercomplex manifold, let $A$ be the H-algebra of q-holomorphic functions on $M$, let $P$ be an H-subalgebra of $A$, and let $Q$ be an AH-submodule of $P$ that generates $P$ as an H-algebra, in the sense of $\$ 2.4$. From $\$ 3.2$, if $m \in M$, then $m$ defines an $\mathbb{H}$-linear map $\theta_{m}: A \rightarrow \mathbb{H}$ such that $\theta_{m} \in A^{\dagger}$. Now $\mathbb{H}$ is itself an H-algebra in the obvious way, and it is easy to see that in fact $\theta_{m}$ is an $\mathrm{H}$-algebra morphism. Therefore $\left.\theta_{m}\right|_{P}: P \rightarrow \mathbb{H}$ is also an H-algebra morphism, and $\left.\theta_{m}\right|_{P} \in P^{\dagger}$.

Conversely, suppose $\theta \in P^{\dagger}$, so that $\theta: P \rightarrow \mathbb{H}$ is an $\mathbb{H}$-linear map. From Definition 2.1.2 we calculate that $\theta$ is an H-algebra morphism if and only if $\theta$ satisfies the quadratic equation $\mu_{P}^{\dagger}(\theta)=\lambda_{P, P}(\theta \otimes \theta)$ in $\left(P \otimes_{\mathbb{H}} P\right)^{\dagger}$, where $\mu_{P}^{\dagger}: P^{\dagger} \rightarrow\left(P \otimes_{\mathbb{H}} P\right)^{\dagger}$ is the dual of the multiplication map $\mu_{P}$, and $\lambda_{P, P}: P^{\dagger} \otimes P^{\dagger} \rightarrow\left(P \otimes_{\mathbb{H}} P\right)^{\dagger}$ is defined in $\S 1.1$.

Suppose $\theta_{1}, \theta_{2}: P \rightarrow \mathbb{H}$ are H-algebra morphisms, and that $\left.\theta_{1}\right|_{Q}=\left.\theta_{2}\right|_{Q}$. Because $Q$ generates $P$, it is easy to see that $\theta_{1}=\theta_{2}$. Hence, H-algebra morphisms from $P$ are determined by their restrictions to $Q$. As $\theta_{1} \in P^{\dagger}$, we have $\left.\theta_{1}\right|_{Q} \in Q^{\dagger}$. The principal case we have in mind is that $P$ is a 'polynomial growth' $\mathrm{H}$-subalgebra as in Definition 3.2.6, and $Q$ is finite-dimensional. By restricting to $Q$ we can work in a finite-dimensional situation. Motivated by the above, we make the following definition.

Definition 3.4.1 Let $P$ be an H-algebra, and $Q$ an AHI-submodule of $P$ that generates $P$. Define $M_{P, Q}$ by

$$
M_{P, Q}=\left\{\left.\theta\right|_{Q}: \theta \in P^{\dagger}, \quad \mu_{P}^{\dagger}(\theta)=\lambda_{P, P}(\theta \otimes \theta)\right\},
$$

so that $M_{P, Q}$ is a closed subset of $Q^{\dagger}$. Now suppose that $M$ is a hypercomplex manifold, and $P$ an $\mathrm{H}$-subalgebra of the $\mathrm{H}$-algebra $A$ of q-holomorphic functions on $M$. For each $m \in M, \theta_{m}: A \rightarrow \mathbb{H}$ is an H-algebra morphism, and so $\left.\theta_{m}\right|_{Q}$ lies in $M_{P, Q}$. Define a map $\pi_{P, Q}: M \rightarrow M_{P, Q}$ by $\pi_{P, Q}(m)=\left.\theta_{m}\right|_{Q}$.

Lemma 3.4.2 Suppose $Q$ is finite-dimensional. Then $M_{P, Q}$ is an affine real algebraic subvariety of $Q^{\dagger}$, that is, it is the zeros of a finite collection of polynomials on $Q^{\dagger}$.

Proof. By Lemma 2.4.4 there is an H-algebra morphism $\phi_{Q}: F^{Q} \rightarrow P$, and as $Q$ generates $P, \phi_{Q}$ is surjective. Let $I \subset F^{Q}$ be the kernel of $\phi_{Q}$. As $F^{Q}$ is a free $\mathrm{H}$-algebra, each element $x$ of $Q^{\dagger}$ defines a unique $\mathrm{H}$-algebra morphism $\theta_{x}: F^{Q} \rightarrow \mathbb{H}$, that restricts to $x$ on $Q$. Clearly, if $x \in Q^{\dagger}$, then $x \in M_{P, Q}$ if and only if $I \subset \operatorname{Ker} \theta_{x}$. 
For each $y \in F^{Q}$, define a function $\psi_{y}: Q^{\dagger} \rightarrow \mathbb{H}$ by $\psi_{y}(x)=\theta_{x}(y)$ for $x \in Q^{\dagger}$. It is easy to see that if $y \in F_{k}^{Q}$, then $\psi_{y}$ is an $\mathbb{H}$-valued polynomial on $Q^{\dagger}$ of degree at most $k$. But $x \in M_{P, Q}$ if and only if $\psi_{y}(x)=0$ for each $y \in I$. Thus $M_{P, Q}$ is the zeros of a collection of polynomials on $Q^{\dagger}$. By Hilbert's Basis Theorem, we can choose a finite number of polynomials, which define $M_{P, Q}$.

Our aim is to recover $M$ and its hypercomplex structure from $P$ and $Q$. If we are lucky, $\pi_{P, Q}$ will be (locally) a bijection, so that $M_{P, Q}$ gives us the manifold $M$, at least as a set. Then we can try and use $P$ to define a hypercomplex structure on $M_{P, Q}$. However, there are a number of ways in which this process could fail.

- $M_{P, Q}$ might not be a submanifold of $Q^{\dagger}$.

- $\pi_{P, Q}$ might not be (locally) injective.

- $\pi_{P, Q}$ might not be (locally) surjective.

- Even if $M_{P, Q}$ is a submanifold of $Q^{\dagger}$ and $\pi_{P, Q}$ is a diffeomorphism, we may be unable to define the hypercomplex structure on $M_{P, Q}$, because $P$ may contain only partial information about the structure.

Unfortunately, all of these possibilities do occur, and examples will be given in $\$ 4.2$. Sometimes the hypercomplex manifold $M$ can be reconstructed, and sometimes not; for polynomial growth $\mathrm{H}$-algebras $P$ and complete $M$, this seems to depend only on the asymptotic behaviour of $M$ at infinity. Next we shall explain how, in good cases, the hypercomplex structure of $M$ may be recovered from $P$.

Lemma 3.4.3 Let $M$ be a hypercomplex manifold of dimension $4 k$, $A$ the $H$-algebra of q-holomorphic functions on $M, P$ an $H$-subalgebra of $A$, and $Q$ an AH-submodule of $P$ generating $P$. Let $m \in M$. Then the derivative of $\pi_{P, Q}$ at $m$ gives a linear map $d_{m} \pi_{P, Q}: T_{m} M \rightarrow Q^{\dagger}$.

Regard $Q$ as a vector space of $\mathbb{H}$-valued functions on $Q^{\dagger}$. Pulling these functions back to $T_{m} M$ using $d_{m} \pi_{P, Q}$ gives a linear map $\left(d_{m} \pi_{P, Q}\right)^{*}: Q \rightarrow$ $T_{m}^{*} M \otimes \mathbb{H}$. Define $V_{m}=\operatorname{Im}\left(\left(d_{m} \pi_{P, Q}\right)^{*}\right)$, so that $V_{m}$ is a linear subspace of $T_{m}^{*} M \otimes \mathbb{H}$. Then $\operatorname{dim} V_{m} \leq 12 k$, and $V_{m}$ determines the hypercomplex structure on $T_{m} M$ if and only if $\operatorname{dim} V_{m}=12 k$. 
Proof. Let $v \in V_{m}$, and write $v=v_{0} \otimes 1+v_{1} \otimes i_{1}+v_{2} \otimes i_{2}+v_{3} \otimes i_{3}$. Now $v$ is the first derivative at $m$ of some element of $Q$, which is a q-holomorphic function on $M$. By definition of q-holomorphic function, it follows that $v_{0}+$ $I_{1} v_{1}+I_{2} v_{2}+I_{3} v_{3}=0$, where $I_{1}, I_{2}, I_{3}$ are the complex structures on $T_{m}^{*} M$. Let $W_{m} \subset T_{m}^{*} M \otimes \mathbb{H}$ be the subspace of elements $w_{0} \otimes 1+w_{1} \otimes i_{1}+w_{2} \otimes i_{2}+w_{3} \otimes i_{3}$ satisfying $w_{0}+I_{1} w_{1}+I_{2} w_{2}+I_{3} w_{3}=0$. Then $W_{m}$ has dimension $12 k$, and $V_{m} \subset W_{m}$, so $\operatorname{dim} V_{m} \leq 12 k$, as we have to prove.

If $\operatorname{dim} V_{m}=12 k$, then $V_{m}=W_{m}$. But $W_{m}$ determines $I_{1}, I_{2}$ and $I_{3}$. (For instance, $w_{1}=I_{1} w_{0}$ if and only if $w_{0} \otimes 1+w_{1} \otimes i_{1} \in W_{m}$, so $W_{m}$ determines $I_{1}$.) Thus if $\operatorname{dim} V_{m}=12 k$, then $V_{m}$ determines the hypercomplex structure on $T_{m} M$. Now $W_{m}$ is an $\mathbb{H}$-submodule of $T_{m}^{*} M \otimes \mathbb{H}$, and it can be shown that if $W$ is an $\mathbb{H}$-submodule of $T^{*} M \otimes \mathbb{H}$ such that $W \neq W_{m}$, $\operatorname{dim} W=12 k$, and $W$ is close to $W_{m}$, then $W$ determines a different hypercomplex structure. It is easy to see that if $\operatorname{dim} V_{m}<12 k$, then we may choose such a $W$ with $V_{m} \subset W$. Thus $V_{m}$ is consistent with two different hypercomplex structures. Therefore $V_{m}$ determines the hypercomplex structure on $T_{m} M$ if and only if $\operatorname{dim} V_{m}=12 k$.

Corollary 3.4.4 In the situation of Lemma 3.4.3, suppose that $\operatorname{dim} V_{m}=$ $12 k$, and that $\pi_{P, Q}$ is surjective near $m$. Then $P$ determines the hypercomplex structure of $M$ near $m$.

Proof. As $\operatorname{dim} V_{m}=12 k$, we deduce that $d_{m} \pi_{P, Q}$ is injective. Together with the surjectivity assumption, this implies that $\pi_{P, Q}$ is a diffeomorphism near $m$. Also, $\operatorname{dim} V_{n}=12 k$ for $n$ near $m$. The conclusion follows from the lemma.

In the special case $\operatorname{dim} M=4$, we can give a more convenient condition for $P$ to determine the hypercomplex structure of $M$.

Proposition 3.4.5 Let $M$ be a hypercomplex manifold of dimension 4, A the $H$-algebra of q-holomorphic functions on $M, P$ an $H$-subalgebra of $A$, and $Q$ an $\mathrm{A} \mathbb{H}$-submodule of $P$ generating $P$. Suppose that $Q$ is stable, that $M_{P, Q}$ is a submanifold of $Q^{\dagger}$, and that $\pi_{P, Q}$ is a (local) diffeomorphism. Then $P$ determines the (local) hypercomplex structure of $M$.

Proof. Because of Corollary 3.4.4, it is sufficient to show that $\operatorname{dim} V_{m}=12$ for each $m \in M$. Suppose for a contradiction that $m \in M$ and $\operatorname{dim} V_{m}<12$. 
As $\pi_{P, Q}$ is a diffeomorphism, $d_{m} \pi_{P, Q}$ is injective. Let $R=\operatorname{Ker}\left(\left(d_{m} \pi_{P, Q}\right)^{*}\right)$, so that $R$ is a proper AII-submodule of $Q$, and $V_{m} \cong Q / R$. Since $d_{m} \pi_{P, Q}$ is injective and $\operatorname{dim} M=4$, we have $\operatorname{dim} Q^{\dagger}=\operatorname{dim} R^{\dagger}+4$.

Now $V_{m}$ is an $\mathbb{H}$-module with $\operatorname{dim} V_{m}<12$, so $\operatorname{dim} V_{m} \leq 8 . \operatorname{But} \operatorname{dim} Q=$ $\operatorname{dim} R+\operatorname{dim} V_{m}$, so $\operatorname{dim} Q \leq \operatorname{dim} R+8$. By Definition 1.2.3, using $\operatorname{dim} Q^{\dagger}=$ $\operatorname{dim} R^{\dagger}+4$, we see that the virtual dimension of $R$ is greater or equal to that of $Q$. Using the stability of $Q$, we then prove that for each nonzero $q \in \mathbb{I}$, the image of id $\otimes_{\mathbb{H}} \chi_{q}: Q \otimes_{\mathbb{H}} X_{q} \rightarrow Q$ lies in $R$, and as $R$ is a proper AHI-submodule, this contradicts the semistability of $Q$. Thus $\operatorname{dim} V_{m}=12$ for all $m \in M$, and the proposition is complete.

The author has not found a satisfactory analogue of this proposition for higher dimensions. One can also consider the problem of reconstructing a hyperkähler manifold from an HP-algebra. It can be solved easily using a similar approach.

\subsection{Asymptotically conical hyperkähler manifolds}

In this section we shall define an interesting class of hyperkähler manifolds, and conjecture some theory about them.

Definition 3.5.1 Let $N$ be a compact manifold of dimension $4 n-1$, and set $C=N \times(0, \infty)$. Let $t: C \rightarrow(0, \infty)$ be the projection to the second factor. Let $v$ be the vector field $t \partial / \partial t$ on $C$. Suppose $C$ has a hyperkähler structure, with metric $g$ and complex structures $I_{1}, I_{2}, I_{3}$. We say that $C$ is a hyperkähler cone if $v$ is a Killing vector of $I_{1}, I_{2}, I_{3}$, and $g=t^{2} h+d t^{2}$, where $h$ is a Riemannian metric on $N$ that is independent of $t$.

Definition 3.5.2 Let $C$ be a hyperkähler cone. Let $f$ be a q-holomorphic function on $C$, and let $k \geq 0$ be an integer. We say $f$ is homogeneous of degree $k$ if $f=t^{k} f_{N}$, where $f_{N}$ is an $\mathbb{H}$-valued function on $N$, independent of $t$. Define $B^{k}$ to be the AHH-module of q-holomorphic functions on $C$ that are homogeneous of degree $k$. Define $B=\bigoplus_{k=0}^{\infty} B^{k}$. Then $B$ is an AHIsubmodule of the $\mathrm{H}$-algebra $A_{C}$ of q-holomorphic functions on $C$. Clearly, $B$ is a graded $\mathrm{H}$-algebra.

Here are some basic properties of hyperkähler cones. The proof is left to the reader.

Lemma 3.5.3 Let $C$ be a hyperkähler cone. Then the vector fields $v, I_{1} v$, $I_{2} v, I_{3} v$ generate an action of the Lie algebra $\mathbb{R} \oplus \mathfrak{s} u(2)$ on $C$, and exponen- 
tiating them gives an action of the Lie group $\mathbb{R} \times S U(2)$ on $C$. Let $c \in C$, and let $\mathcal{O}_{c}$ be the orbit of $c$ under $\mathbb{R} \times S U(2)$. Then $\mathcal{O}_{c}$ is a hyperkähler submanifold of $C$, and is isomorphic as a hyperkähler manifold to $(\mathbb{H} \backslash\{0\}) / \Gamma$, for some finite subgroup $\Gamma \subset S U(2)$.

Lemma 3.5.4 Let $C$ be a hyperkähler cone. Then the graded H-algebra $B$ of Definition 3.5.9 is equal to the filtered H-algebra $P_{C}$ of q-holomorphic functions of polynomial growth on $C$.

Proof. Let $f$ be a q-holomorphic function on $C$, of polynomial growth of degree $k$. Let $c \in C$. By Lemma 3.5.3, $\mathcal{O}_{c}$ is isomorphic to $(\mathbb{H} \backslash\{0\}) / \Gamma$, so that the universal cover $\tilde{\mathcal{O}}_{c}$ is isomorphic to $\mathbb{H} \backslash\{0\}$. Restricting $f$ to $\mathcal{O}_{c}$ and lifting to $\tilde{\mathcal{O}}_{c}$, the result is a q-holomorphic function on $\mathbb{H} \backslash\{0\}$, of polynomial growth of degree $k$.

Now all such functions are in fact polynomials of degree $k$ on $\mathbb{H}$, by a classical result about harmonic functions of polynomial growth on $\mathbb{R}^{n}$. Therefore, we may decompose $f$ on $C$ into a sum of homogeneous polynomials on each $\mathcal{O}_{c}$. Clearly, these homogeneous pieces lie in $B^{j}$ for $j \leq k$, so $f \in \bigoplus_{j=0}^{k} B^{j}$. We have shown that if $f \in P_{C}$, then $f \in B$, and $P_{C} \subset B$. But the inclusion $B \subset P_{C}$ is immediate, so $B=P_{C}$. Clearly, the filtrations on $B$ and $P_{C}$ agree, and the lemma is complete.

Next, we shall define asymptotically conical hyperkähler manifolds.

Definition 3.5.5 Let $M$ be a complete hyperkähler manifold of dimension $4 n$, with metric $g^{M}$ and complex structures $I_{1}^{M}, I_{2}^{M}, I_{3}^{M}$. Let $C=N \times$ $(0, \infty)$ be a hyperkähler cone of dimension $4 n$, with metric $g^{C}$ and complex structures $I_{1}^{C}, I_{2}^{C}, I_{3}^{C}$. Let $K$ be a compact subset of $M$. Then $M \backslash K$ and $N \times(1, \infty)$ are submanifolds of $M$ and $C$. Suppose that $\Phi: M \backslash K \rightarrow$ $N \times(1, \infty)$ is a diffeomorphism. Let $\nabla$ be the Levi-Civita connection on $C$, and $l$ be a positive integer.

We say that $M$ is asymptotically conical, or $A C$ to order $l$, if

$$
\begin{gathered}
\Phi_{*}\left(g^{M}\right)=g^{C}+O\left(t^{-l}\right), \quad \nabla\left(\Phi_{*}\left(g^{M}\right)\right)=O\left(t^{-l-1}\right), \\
\nabla^{2}\left(\Phi_{*}\left(g^{M}\right)\right)=O\left(t^{-l-2}\right), \quad \text { and } \\
\Phi_{*}\left(I_{j}^{M}\right)=I_{j}^{C}+O\left(t^{-l}\right), \quad \nabla\left(\Phi_{*}\left(I_{j}^{M}\right)\right)=O\left(t^{-l-1}\right), \\
\nabla^{2}\left(\Phi_{*}\left(I_{j}^{M}\right)\right)=O\left(t^{-l-2}\right), \quad \text { for } j=1,2,3 .
\end{gathered}
$$


These equations should be interpreted as follows. Let $T$ be a tensor field on $N \times(1, \infty)$. Then $T=O\left(t^{-k}\right)$ means that $|T| \leq \kappa t^{-k}$ on $N \times(1, \infty)$, where $\kappa$ is a positive constant, and $|$.$| is taken w.r.t. the metric g^{C}$. We call $C$ the asymptotic cone of $M$.

Our aim in the remainder of this section is to explore the structure of the H-algebra of q-holomorphic functions on an AC hyperkähler manifold. We shall now state - but not prove, and I do not know a complete proof - a powerful result relating the q-holomorphic functions on the AC manifold and its asymptotic cone. An incomplete proof will be given shortly, that depends on conjectures to be stated below.

Theorem 3.5.6 Let $M$ be a hyperkähler manifold that is AC of order $l$, with asymptotic cone $C$. Let $B$ be the graded H-algebra of q-holomorphic functions on $C$, defined in Definition 3.5.9. Let $P$ be the filtered H-algebra of q-holomorphic functions on $M$ with polynomial growth. Then $B$ is an $S G H$-algebra, $P$ is an SFH-algebra, and $B$ is isomorphic to $P$ to order $l$, in the sense of Definition 2.3.5. This implies that $B$ is the associated graded H-algebra of $P$, as in Proposition 2.3.6.

We shall state two conjectures, and then prove the theorem assuming these. Before making the conjectures, we shall define tensor fields of polynomial growth on AC hyperkähler manifolds.

Definition 3.5.7 Let $M$ be an AC hyperkähler manifold, with asymptotic cone $C$. Let $T$ be a smooth tensor field or function on $M$, and $k$ be an integer. Then the expression $|T|=O\left(t^{k}\right)$ means that $|T| \leq \kappa \Phi^{*}(t)^{k}$ on $M \backslash K$ for some positive constant $\kappa$, where $|$.$| is taken w.r.t. the metric g^{M}$, and $\Phi, K, t$ and $g^{M}$ are as in Definition 3.5.5.

With this definition we can state the first conjecture.

Conjecture 3.5.8 Let $M$ be a hyperkähler manifold that is AC of order $l \geq 1$, and let $k \geq-1$ be an integer. Let $x: M \rightarrow \mathbb{H}$ be a smooth function, so that $D(x)$ is a 1 -form on $M$, where $D$ is the operator of $\$ 3.1$. Suppose that $\nabla^{a} D(x)=O\left(t^{k-a-1}\right)$ for $a=0,1,2$, where $\nabla$ is the Levi-Civita connection on $M$. Then there exists a smooth function $y: M \rightarrow \mathbb{H}$ such that $D(x)=D(y)$, and $y=O\left(t^{k}\right)$. Moreover, if $x$ takes values in $\mathbb{I}$, then $y$ can be chosen to take values in $\mathbb{I}$.

This conjecture is a result in analysis, and I believe that it can be proved using existing mathematical ideas and techniques. Much work has been done 
on a similar problem, that of studying the harmonic functions of polynomial growth on an asymptotically flat manifold. Some relevant examples are 22, Th. 9.2, p. 76], [4, Th. 1.17, p. 674], and in particular the proof of Theorem 3.1 in [1, p. 678]. See also [23], in which Li and Yau study holomorphic functions of subquadratic growth on an asymptotically flat Kähler manifold.

These results give strong relations between the harmonic functions of polynomial growth on a Riemannian manifold $M$ asymptotic to $\mathbb{R}^{n}$, and the harmonic polynomials on $\mathbb{R}^{n}$. Conjecture 3.5 .8 is modelled on them. I believe that generalizing from asymptotically flat to asymptotically conical manifolds should be easy. The problems will come from dealing with the operator $D$ rather than $\Delta$. One possible tool to use here is Baston's elliptic complex of operators [5, p. 43-44] resolving $D$.

Conjecture 3.5.9 Let $C$ be a hyperkähler cone. Then the graded H-algebra $B$ of Definition 3.5.2 is an SGH-algebra.

In fact, the author's calculations suggest that hyperkähler cones $C$ always have $B^{2 k} \cong \mathbb{R}^{a} \otimes S_{\mathbb{H}}^{k} Y$, where $a \geq 0$ is an integer depending on $k$ and $Y$ is the AHI-module of $\$ 2.2$, and that most $C$ also have $B^{2 k+1}=\{0\}$. Conjecture 3.5 .9 would follow immediately from this. Assuming Conjectures 3.5 .8 and 3.5.9, we will now prove Theorem 3.5.6.

Sketch proof of Theorem 3.5.0. Let $j \geq 0$ be an integer. We shall construct a linear map $\phi_{j}: B_{j} / B_{j-l} \rightarrow P_{j} / P_{j-l}$. Let $b \in B_{j}$. Then $b$ is a q-holomorphic function on $C$, with polynomial growth of degree $j$. With a partition of unity, one may construct a smooth, $\mathbb{H}$-valued function $x$ on $M$, such that $\Phi_{*}(x)=b$ on $N \times(2, \infty) \subset C$. Using equations (18) and (19) and the fact that $\nabla^{a} b=O\left(t^{j-a}\right)$ on $C$ for large $t$ (this is easily proved), it can be shown that $\nabla^{a} D(x)=O\left(t^{j-l-a-1}\right)$ for $a=0,1,2$ on $M$.

Putting $k=j-l$, or $k=-1$ if $l>j+1$, we may apply Conjecture 3.5.8. It shows that there exists a smooth, $\mathbb{H}$-valued function $y$ on $M$ with $y=O\left(t^{j-l}\right)$ or $y=O\left(t^{-1}\right)$ respectively, such that $D(x)=D(y)$ on $M$. Therefore, $D(x-y)=0$, so $x-y$ is q-holomorphic on $M$. Clearly, $x-y$ has polynomial growth of order $j$, so $x-y \in P_{j}$. Now $y$ may not be unique, but since $y=O\left(t^{j-l}\right)$ or $O\left(t^{-1}\right)$, any two solutions $y$ differ by an element of $P_{j-l}$ or $P_{-1}=\{0\}$. Therefore, $x-y+P_{j-l}$ is a well-defined element of $P_{j} / P_{j-l}$, depending only on $b$.

Define a map $\phi_{j}: B_{j} / B_{j-l} \rightarrow P_{j} / P_{j-l}$ by $\phi_{j}\left(b+B_{j-l}\right)=x-y+P_{j-l}$. Then $\phi_{j}$ is a well-defined $\mathbb{H}$-linear map. Suppose that $b \in B_{j}^{\prime}$. Then $b$ takes values in $\mathbb{I}$. So $x$ takes values in $\mathbb{I}$, and by Conjecture 3.5 .8 we may choose $y$ to take 
values in $\mathbb{I}$. Thus $x-y \in P_{j}^{\prime}$, and $\phi_{j}$ maps $B_{j}^{\prime} / B_{j-l}^{\prime}$ to $P_{j}^{\prime} / P_{j-l}^{\prime}$. Therefore $\phi_{j}$ is an AHI-morphism, provided $B_{j} / B_{j-l}$ and $P_{j} / P_{j-l}$ are AHH-modules. With a little more work, one shows that $\phi_{j}$ is an AHI-isomorphism.

It remains to verify the conditions of Definition 2.3.5. From the definition of $\phi_{j}$ it immediately follows that $\phi_{j}$ takes $B_{k} / B_{j-l}$ to $P_{k} / P_{j-l}$ for $j-l \leq k \leq j$, and also that $\phi_{j}=\phi_{j+1}$ on $B_{j} / B_{j-l+1}$. By Conjecture 3.5.9, $B_{j} / B_{j-l}$ is a stable AHI-module, so $P_{j} / P_{j-l}$ is also a stable AHH-module. The equation

$\mu_{j k l}^{P} \circ\left(\phi_{j} \otimes_{\mathbb{H}} \phi_{k}\right)=\phi_{j+k} \circ \mu_{j k l}^{B}$ comes naturally out of the construction of $\phi_{j}$. Thus $B$ is isomorphic to $P$ to order $l$, by definition. Finally, it follows easily that $B$ is the associated graded $\mathrm{H}$-algebra of $P$.

\section{Examples, applications and conclusions}

This chapter was difficult to write, because of the many examples, little bits of theory, and quaternionic versions of this and that which begged to be included. For reasons of time and space I have been ruthless, discussing a few topics only, and not in great depth. However, I think that one could easily fill another paper the length of this one with interesting material.

Section 4.1 finds the HP-algebra of q-holomorphic functions of polynomial growth on $\mathbb{H}$, in a series of simple steps, as an example. In $\$ 4.2$ we give examples of how the programme of $\$ 3.4$ (to recover a hypercomplex manifold from its H-algebra) may fail. Then $\$ 4.3$ shows how to make HL-algebras and HP-algebras out of ordinary Lie algebras. This device is applied in $\$ 4.4$, which is about hyperkähler manifolds with symmetries. The high point of $\$ 4.4$ is a (conjectural) algebraic method to explicitly construct 'coadjoint orbit' hyperkähler manifolds, using HP-algebras.

In $\S$ t.5 we look at at the simplest nontrivial hyperkähler manifold - the Eguchi-Hanson space. It fits into our theory both as an AC hyperkähler manifold, and as a 'coadjoint orbit'. A careful investigation of the H-algebra and its deformations reveals a surprise: an unexpected family of singular hypercomplex structures with remarkable properties. Section 4.6 interprets self-dual connections, or 'instantons', over a hypercomplex manifold, as modules over its H-algebra. Finally, $\$$. .7 concludes the paper with some research problems. 


\subsection{Q-holomorphic functions on $\mathbb{H}$}

Let $\mathbb{H}$ have real coordinates $\left(x_{0}, \ldots, x_{3}\right)$, so that $\left(x_{0}, \ldots, x_{3}\right)$ represents $x_{0}+$ $x_{1} i_{1}+x_{2} i_{2}+x_{3} i_{3}$. Now $\mathbb{H}$ is naturally a hypercomplex manifold with complex structures given by $I_{1} d x_{2}=d x_{3}, I_{2} d x_{3}=d x_{1}, I_{3} d x_{1}=d x_{2}$ and $I_{j} d x_{0}=$ $d x_{j}$, for $j=1,2,3$. The study of q-holomorphic functions on $\mathbb{H}$ is called quaternionic analysis, and is surveyed in [27].

Example 4.1.1 First we shall determine the AHI-module $U$ of all linear q-holomorphic functions on $\mathbb{H}$. Let $q_{0}, \ldots, q_{3} \in \mathbb{H}$, and define $u=q_{0} x_{0}+$ $\cdots+q_{3} x_{3}$ as an $\mathbb{H}$-valued function on $\mathbb{H}$. A calculation shows that $u$ is q-holomorphic if and only if $q_{0}+q_{1} i_{1}+q_{2} i_{2}+q_{3} i_{3}=0$. It follows that $U \cong \mathbb{H}^{3}$. Also, $U^{\prime}$ is the vector subspace of $U$ with $q_{j} \in \mathbb{I}$ for $j=0, \ldots, 3$. Let us identify $U$ with $\mathbb{H}^{3}$ explicitly by taking $\left(q_{1}, q_{2}, q_{3}\right)$ as quaternionic coordinates. Then

$$
U^{\prime}=\left\{\left(q_{1}, q_{2}, q_{3}\right) \in \mathbb{H}^{3}: q_{j} \in \mathbb{I} \text { for } j=1,2,3 \text { and } q_{1} i_{1}+q_{2} i_{2}+q_{3} i_{3} \in \mathbb{I}\right\} .
$$

Thus $U^{\prime} \cong \mathbb{R}^{8}$, and $\operatorname{dim} U=4 j, \operatorname{dim} U^{\prime}=2 j+r$ with $j=3$ and $r=2$, so the virtual dimension of $U$ is 2 . This is because $\mathbb{H} \cong \mathbb{C}^{2}$, so the complex dimension of $\mathbb{H}$ is 2 . It is easy to see that $U$ is a stable AH-module.

Example 4.1.2 Let $k \geq 0$ be an integer, and let $U^{(k)}$ be the AHH-module of q-holomorphic functions on $\mathbb{H}$ that are homogeneous polynomials of degree $k$. We shall determine $U^{(k)}$. Write $A$ for the H-algebra of q-holomorphic functions on $\mathbb{H}$, and $\mu_{A}: A \otimes_{\mathbb{H}} A \rightarrow A$ for the multiplication map. By Example 4.1.1, $U^{(1)}=U \subset A$. Thus $\mu_{A}$ induces an AHI-morphism $\mu_{A}: U \otimes_{\mathbb{H}} U \rightarrow A$, and composing $\mu_{A} k-1$ times gives an AHI-morphism $\mu_{A}^{k-1}: \bigotimes_{\mathbb{H}}^{k} U \rightarrow A$. Clearly, $\operatorname{Im} \mu_{A}^{k-1} \subset U^{(k)}$. Also, $\mu_{A}^{k-1}$ is symmetric in the $k$ factors of $U$, so it makes sense to restrict to $S_{\mathbb{H}}^{k} U$.

Thus we have constructed an AHH-morphism $\mu_{A}^{k-1}: S_{\mathbb{H}}^{k} U \rightarrow U^{(k)}$. It is easy to show that $\mu_{A}^{k-1}$ is injective on $S_{\mathbb{H}}^{k} U$. By Example 4.1.1, $U$ is stable with $j=$ 3 and $r=2$. Thus Proposition 1.2 .8 shows that $\operatorname{dim} S_{\mathbb{H}}^{k} U=2(k+1)(k+2)$. But Sudbery [27, Th. 7, p. 217] shows that $\operatorname{dim} U^{(k)}=2(k+1)(k+2)$. It follows that $\mu_{A}^{k-1}$ is an isomorphism, and $U^{(k)} \cong S_{\mathbb{H}}^{k} U$.

The interpretation of Example 4.1.2 is simple. If $V$ is the linear polynomials on some vector space, then $S^{k} V$ is the homogeneous polynomials of degree $k$. Here we have a quaternionic analogue of this, replacing $S^{k}$ by $S_{\mathbb{H}}^{k}$. We have found an elegant construction of the spaces $U^{(k)}$, important 
in quaternionic analysis, that gives insight into their algebraic structure and dimension.

Example 4.1.3 Let us consider the filtered H-algebra $P$ of q-holomorphic functions of polynomial growth on $\mathbb{H}$, as in $\$ 3.2$. Clearly, the functions in $U^{(k)}$ have polynomial growth of order $k$, so that $U^{(k)} \subset P_{k} \subset P$. Thus $\bigoplus_{j=0}^{k} U^{(j)} \subset P_{k}$, and $\bigoplus_{j=0}^{\infty} U^{(j)} \subset P$. Now it is a well-known result in complex analysis that all holomorphic functions on $\mathbb{C}$ of polynomial growth, are polynomials. The obvious analogue of this is that all q-holomorphic functions on $\mathbb{H}$ of polynomial growth are sums of elements of $U^{(k)}$.

This is in fact true, and can be proved using the theory in [27]. Therefore $P_{k}=\bigoplus_{j=0}^{k} U^{(j)}$, and $P=\bigoplus_{j=0}^{\infty} U^{(j)}$. But from Example 4.1.2, $U^{(j)}=$ $S_{\mathbb{H}}^{j} U$. Thus $P=\bigoplus_{j=0}^{\infty} S_{\mathbb{H}}^{j} U$. So, by Definition 2.4.3, $P$ is isomorphic to the free algebra $F^{U}$ generated by $U$, with its natural filtration. As $U$ is finitedimensional, $P$ is finitely-generated, and in particular, $P$ is an FGH-algebra in the sense of $\$ 2.4$.

The full H-algebra $A$ of q-holomorphic functions on $\mathbb{H}$ is obtained by completing $P$, by adding in convergent power series. The analytic details are beyond the scope of this paper. Note, however, that because $A$ contains all holomorphic functions on $\mathbb{C}^{2}, A$ is certainly not finitely-generated, so that $P$ has a much simpler structure than $A$. We may generalize this example to the hypercomplex manifold $\mathbb{H}^{n}$. It is easy to see that the H-algebra of qholomorphic functions on $\mathbb{H}^{n}$ of polynomial growth is $F^{n U}$, the free H-algebra generated by $n$ copies of $U$.

Now $\mathbb{H}$ is a hyperkähler manifold, so by Theorem 3.3.4, $A$ and $P$ should be HP-algebras. We shall define the HP-algebra structure on $P$.

Example 4.1.4 We must construct an AHI-morphism $\xi_{P}: P \otimes_{\mathbb{H}} P \rightarrow P \otimes_{\mathbb{H}} Y$. From above $U=U^{(1)} \subset P$, so consider $\xi_{P}: U \otimes_{\mathbb{H}} U \rightarrow P \otimes_{\mathbb{H}} Y$. Since $\xi_{P}$ is antisymmetric, we may restrict to $\Lambda_{\mathbb{H}}^{2} U$. Now $U$ is stable and has $j=3, r=2$, so by Proposition 1.2.8, we have $\operatorname{dim} \Lambda_{\mathbb{H}}^{2} U=8$ and $\operatorname{dim}\left(\Lambda_{\mathbb{H}}^{2} U\right)^{\prime}=5$. But these are the same dimensions as those of the AHI-module $Y$ of Definition 2.2.1. In fact there is a natural isomorphism $\Lambda_{\mathbb{H}}^{2} U \cong Y$. Now $U^{(0)} \cong \mathbb{H}$, so that $U^{(0)} \otimes_{\mathbb{H}} Y \cong Y$. Thus we have AHI-isomorphisms $\Lambda_{\mathbb{H}}^{2} U^{(1)} \cong U^{(0)} \otimes_{\mathbb{H}} Y \cong Y$.

It is easy to show that the restriction of $\xi_{P}$ to $\Lambda_{\mathbb{H}}^{2} U^{(1)}$ gives exactly this isomorphism $\Lambda_{\mathbb{H}}^{2} U^{(1)} \cong U^{(0)} \otimes_{\mathbb{H}} Y$. Thus we have defined $\xi_{P}$ on a generating subspace $U^{(1)}$ for $P$. Using Axiom P2, we may extend $\xi_{P}$ uniquely to all of $P$, because the action of $\xi_{P}$ on the generators defines the whole action. Now $P$ 
is a filtered H-algebra, and $\xi_{P}$ satisfies $\xi_{P}\left(P_{j} \otimes_{\mathbb{H}} P_{k}\right) \subset P_{j+k-2}$. Thus Axiom $\mathrm{PF}$ of $\$ 2.3$ holds, and $P$ is a filtered HP-algebra.

\subsection{Hypercomplex manifolds undetermined by their H-algebras}

In $\$ 3.4$ we explained how, under good conditions, it is possible to reconstruct a hypercomplex manifold from an $\mathrm{H}$-algebra of q-holomorphic functions upon it. Here are three examples where this cannot be done, illustrating different ways in which the reconstruction can fail.

Example 4.2.1 Since $\mathbb{Z} \subset \mathbb{R} \subset \mathbb{H}$, $\mathbb{Z}$ acts on $\mathbb{H}$ by translation, and so $\mathbb{H} / \mathbb{Z} \cong \mathbb{R}^{3} \times \mathcal{S}^{1}$ is a hyperkähler manifold. We shall determine the filtered H-algebra $P$ of q-holomorphic functions on $M=\mathbb{H} / \mathbb{Z}$ of polynomial growth. But $\mathbb{H}$ covers $M$, so any q-holomorphic function of polynomial growth on $M$ lifts to a q-holomorphic function of polynomial growth on $\mathbb{H}$. So by Example 4.1.3, $P$ is the H-subalgebra of $F^{U}$ that is invariant under $\mathbb{Z}$.

Clearly, any polynomial invariant under $\mathbb{Z}$ is also invariant under $\mathbb{R} \subset \mathbb{H}$. Referring to Example 4.1.3, the elements of $U$ invariant under $\mathbb{R}$ are those with $q_{0}=0$. Therefore, the $\mathbb{R}$-invariant polynomials in $U$ are $\left\{\left(q_{1}, q_{2}, q_{3}\right) \in\right.$ $\left.\mathbb{H}^{3}: q_{1} i_{1}+q_{2} i_{2}+q_{3} i_{3}=0\right\} \subset U$. But this is the AHI-module $Y$ of $\$ 2.2$. So, one may show that the H-algebra $P$ of q-holomorphic functions on $M$ of polynomial growth is the free H-algebra $F^{Y}$.

Now consider reconstructing $M$ from $P$, as in $\$ 3.4$. We have $P=F^{Y}$, so we put $Q=Y$. Then $Q^{\dagger}=\mathbb{I}=\mathbb{R}^{3}$. As $P=F^{Q}, M_{P, Q}$ is the whole of $Q^{\dagger}=\mathbb{R}^{3}$. But $M \cong \mathbb{R}^{3} \times \mathcal{S}^{1}$, and the map $\pi_{P, Q}: M \rightarrow M_{P, Q}$ is simply the projection to the first factor $\mathbb{R}^{3} \times \mathcal{S}^{1} \rightarrow \mathbb{R}^{3}$. Therefore, in this case, $M_{P, Q}$ is a manifold, and $\pi_{P, Q}$ is surjective, but not (even locally) injective. Thus, we cannot recover the manifold $M$ from $P$.

Example 4.2.2 Much of Atiyah and Hitchin's book [2] is an in-depth study of a particular complete, noncompact hyperkähler 4-manifold, the 2monopole moduli space $M_{2}^{0}$. As a manifold, $M_{2}^{0}$ is diffeomorphic to a complex line bundle over $\mathbb{R P}^{2}$, and the zero section gives a submanifold $\mathbb{R P}^{2} \subset M_{2}^{0}$. The isometry group of the metric $g$ on $M_{2}^{0}$ is $S O(3)$. This isometry group acts in a nontrivial way on the hyperkähler structure. There is a vector space isomorphism $\left\langle I_{1}, I_{2}, I_{3}\right\rangle \cong \mathfrak{s o}(3)$, that identifies the action of $S O(3)$ on $\left\langle I_{1}, I_{2}, I_{3}\right\rangle$ with the adjoint action on $\mathfrak{s o}(3)$. 
Because $S O(3)$ does not fix the hyperkähler structure, there are no hyperkähler moment maps (see \$4.4). However, there are some Kähler moment maps. Let $v_{1}, v_{2}, v_{3} \in \mathfrak{s o}(3)$ be identified with $I_{1}, I_{2}, I_{3}$ under the isomorphism above. Then $v_{1}, v_{2}, v_{3}$ are Killing vectors of $g$ on $M$. Let $\alpha_{1}, \alpha_{2}, \alpha_{3}$ be the 1 -forms on $M_{2}^{0}$ dual to $v_{1}, v_{2}, v_{3}$ under $g$. Then a brief calculation shows that the 1 -form $I_{j} \alpha_{k}+I_{k} \alpha_{j}$ is closed, for $j, k=1, \ldots, 3$. Since $b^{1}\left(M_{2}^{0}\right)=0$, there exists a unique real function $f_{j k}$ with $d f_{j k}=I_{j} \alpha_{k}+I_{k} \alpha_{j}$, and such that $\int_{\mathbb{R}^{2}} f_{j k} d A=0$.

The $f_{j k}$ form a vector space $S^{2} \mathbb{R}^{3} \cong \mathbb{R}^{6}$ of real functions on $M_{2}^{0}$. For $j, k=$ $1,2,3$, let $q_{j k} \in \mathbb{H}$ with $q_{j k}=q_{k j}$, and define $z=\Sigma_{j, k=1}^{3} q_{j k} f_{j k}$. When is $z$ a q-holomorphic function on $M_{2}^{0}$ ? Calculation shows that $z$ is q-holomorphic if and only if $\Sigma_{j=1}^{3} q_{j k} i_{j}=0$ for $k=1,2,3$. Therefore, the AHH-module of q-holomorphic $z$ is

$$
Z=\left\langle f_{22}-f_{33}+2 i_{1} f_{23}, f_{33}-f_{11}+2 i_{2} f_{31}, f_{11}-f_{22}+2 i_{3} f_{12}\right\rangle .
$$

Note that only the trace-free part $S_{0}^{2} \mathfrak{s o}(3) \cong \mathbb{R}^{5}$ appears here. From (20) we find that $Z \cong \mathbb{H}^{3}$ and $Z^{\prime} \cong \mathbb{R}^{7}$, and in fact there is a canonical isomorphism $Z \cong Y \otimes_{\mathbb{H}} Y$, where $Y$ is the AHH-module defined in $\$ 2.2$.

We have found an AHI-module $Z \cong Y \otimes_{\mathbb{H}} Y$ of q-holomorphic functions on $M_{2}^{0}$. By Lemma 2.4.4 there is an H-algebra morphism $\phi_{Z}: F^{Z} \rightarrow A$, where $A$ is the H-algebra of q-holomorphic functions on $M_{2}^{0}$. Now Atiyah and Hitchin [2] define the metric on $M_{2}^{0}$ explicitly, using an elliptic integral. Their construction uses transcendental functions, not just algebraic functions. Because of this, it can be shown that the 5 functions $f_{j k}$ used in (20) are algebraically independent. Therefore, there can be no polynomial relations between them, so that $\phi_{Z}$ is injective.

Thus $F^{Z}$ is an H-algebra of q-holomorphic functions on $M_{2}^{0}$. Now $Z^{\dagger} \cong$ $\mathbb{R}^{5}$, and since $F^{Z}$ is free, $M_{F^{Z}, Z}$ is the whole of $Z^{\dagger}$. Thus $\pi_{F^{Z}, Z}$ maps $M_{2}^{0}$ to $\mathbb{R}^{5}$. It can be shown that $\pi_{F^{Z}, Z}$ is generically injective, but because of the dimensions $\pi_{F}, Z$ cannot be surjective. Thus we cannot recover the manifold $M_{2}^{0}$ from $F^{Z}$. I claim that $F^{Z}$ is in fact the whole $\mathrm{H}$-algebra of q-holomorphic functions of polynomial growth on $M_{2}^{0}$.

Example 4.2.3 Let $k \geq 2$, and let $M=\mathbb{H}^{k}$. Then by Example 4.1.3, the H-algebra of q-holomorphic functions of polynomial growth on $M$ is $F^{k U}$. Now $U \cong \mathbb{H}^{3}$, so $k U \cong \mathbb{H}^{3 k}$. It can be shown that if $k \geq 2$, then the generic $\mathbb{H}$-submodule $Q \cong \mathbb{H}^{3 k-1}$ of $k U$ is a stable AHH-module with $Q^{\prime} \cong \mathbb{R}^{8 k-4}$ and 
$Q^{\dagger}=\mathbb{R}^{4 k}$. Let $Q$ be such an AHH-submodule, and let $P=F^{Q} \subset F^{U}$. Then $P$ is an H-algebra of q-holomorphic functions on $M=\mathbb{H}^{k}$.

Consider reconstructing $M$ and its hypercomplex structure from $P$, as in \$3.4. Since $P$ is free, $M_{P, Q}$ is the whole of $Q^{\dagger}$, which is $\mathbb{R}^{4 k}$. But $M \cong \mathbb{R}^{4 k}$, and in fact $\pi_{P, Q}: M \rightarrow M_{P, Q}$ is a diffeomorphism, the identity. However, in Lemma 3.4 .3 we have $V_{m} \cong Q$ for each $m \in M$, so that $\operatorname{dim} V_{m}=12 k-$ 4. Thus, the lemma shows that the hypercomplex structure of $M$ cannot be recovered from $P$. This means that $P$ gives full information about the manifold $M$, but only partial information about the hypercomplex structure.

\subsection{HL-algebras and HP-algebras}

Here is a simple construction of HL-algebras.

Example 4.3.1 Let $\mathfrak{g}$ be a Lie algebra. Then the Lie bracket $[$,$] on \mathfrak{g}$ gives a linear map $\lambda: \mathfrak{g} \otimes \mathfrak{g} \rightarrow \mathfrak{g}$, such that $\lambda(x \otimes y)=[x, y]$ for $x, y \in \mathfrak{g}$. Let $Y$ be the AHI-module defined in $\delta 2.2$, and define $A_{\mathfrak{g}}$ to be the AHI-module $\mathfrak{g} \otimes Y$. Then $A_{\mathfrak{g}} \otimes_{\mathbb{H}} A_{\mathfrak{g}} \cong(\mathfrak{g} \otimes \mathfrak{g}) \otimes\left(Y \otimes_{\mathbb{H}} Y\right)$ and $A_{\mathfrak{g}} \otimes_{\mathbb{H}} Y \cong \mathfrak{g} \otimes\left(Y \otimes_{\mathbb{H}} Y\right)$. Define a linear map $\xi_{A_{\mathfrak{g}}}: A_{\mathfrak{g}} \otimes_{\mathbb{H}} A_{\mathfrak{g}} \rightarrow A_{\mathfrak{g}} \otimes_{\mathbb{H}} Y$ by $\xi_{A_{\mathfrak{g}}}=\lambda \otimes$ id, as a map from $(\mathfrak{g} \otimes \mathfrak{g}) \otimes\left(Y \otimes_{\mathbb{H}} Y\right)$ to $\mathfrak{g} \otimes\left(Y \otimes_{\mathbb{H}} Y\right)$. It is easy to show that $\xi_{A_{\mathfrak{g}}}$ satisfies Axiom P1 of \$2.2, using the Jacobi identity for $\mathfrak{g}$ to prove part (iii). Therefore, $A_{\mathfrak{g}}$ is an HL-algebra, by Definition 2.2.2.

Example 4.3.2 Example 1.1.4 constructed a filtered HP-algebra $P$. Because $\xi_{P}\left(P_{j} \otimes_{\mathbb{H}} P_{k}\right) \subset P_{j+k-2}$, each of $P_{0}, P_{1}$ and $P_{2}$ are closed under $\xi_{P}$. Therefore, restricting $\xi_{P}$ to $P_{0}, P_{1}$ and $P_{2}$ gives them the structure of HL-algebras. Now $P_{0} \cong \mathbb{H}$ and $\xi_{P}$ is zero on $P_{0}$, but $P_{1} \cong \mathbb{H} \oplus U, P_{2} \cong \mathbb{H} \oplus U \oplus S_{\mathbb{H}}^{2} U$, which are both finite-dimensional, and $\xi_{P}$ is nontrivial on both. It is easy to see that none of these is of the form $\mathfrak{g} \otimes Y$. Thus, there exist nontrivial, finitedimensional HL-algebras that do not arise from Example 4.3.1.

The next example is an aside about Lie and Poisson algebras.

Example 4.3.3 Let $\mathfrak{g}$ be a Lie algebra. The symmetric algebra $S(\mathfrak{g})=$ $\bigoplus_{k=0}^{\infty} S^{k} \mathfrak{g}$ of $\mathfrak{g}$ is a free, commutative algebra generated by $\mathfrak{g}$. Define a bracket $\{\}:, S(\mathfrak{g}) \times S(\mathfrak{g}) \rightarrow S(\mathfrak{g})$ as follows. Let $k, l \geq 0$ be integers. If $x, y \in \mathfrak{g}$, then $x^{k} \in S^{k} \mathfrak{g}$ and $y^{l} \in S^{l} \mathfrak{g}$, and $S^{k} \mathfrak{g}, S^{l} \mathfrak{g}$ are generated by such elements. When $k=0$ or $l=0$ define $\{\}=$,0 on $S^{k} \mathfrak{g} \times S^{l} \mathfrak{g}$, and when 
$k, l>0$ define $\left\{x^{k}, y^{l}\right\}=k l \sigma\left(x^{k-1} \otimes[x, y] \otimes y^{l-1}\right)$. Here $[$,$] is the Lie bracket$ on $\mathfrak{g}$ and $\sigma: S^{k-1} \mathfrak{g} \otimes \mathfrak{g} \otimes S^{l-1} \mathfrak{g} \rightarrow S^{k+l-1} \mathfrak{g}$ is the symmetrization operator, a projection.

This definition extends uniquely to give a bilinear operator $\{\}:, S^{k} \mathfrak{g} \times$ $S^{l} \mathfrak{g} \rightarrow S^{k+l-1} \mathfrak{g}$, so we have found a bilinear bracket $\{$,$\} on S(\mathfrak{g}) \times S(\mathfrak{g})$. It is shown in [0], §1.4] that $\{$,$\} is a Poisson bracket on S(\mathfrak{g})$, so that $S(\mathfrak{g})$ is a Poisson algebra, the Poisson algebra of the Lie algebra $\mathfrak{g}$.

Using Example 4.3.3 as a model, here is a construction of HP-algebras.

Example 4.3.4 Let $A$ be an HL-algebra. Then $\$ 2.4$ defines the free Halgebra $F^{A}$ generated by $A$. To make $F^{A}$ into an HP-algebra, we must give a Poisson bracket $\xi_{F^{A}}$ on $F^{A}$. Let $k, l$ be positive integers, and define a map $\xi_{k, l}: S_{\mathbb{H}}^{k} A \otimes_{\mathbb{H}} S_{\mathbb{H}}^{l} A \rightarrow S_{\mathbb{H}}^{k+l-1} A$ by $\xi_{k, l}=k l \sigma_{\mathbb{H}} \circ\left(\mathrm{id} \otimes_{\mathbb{H}} \xi_{A} \otimes_{\mathbb{H}} \mathrm{id}\right) \circ \iota$, using the sequence of maps

$$
\begin{aligned}
& S_{\mathbb{H}}^{k} A \otimes_{\mathbb{H}} S_{\mathbb{H}}^{l} A \stackrel{\iota}{\rightarrow} S_{\mathbb{H}}^{k-1} A \otimes_{\mathbb{H}} A \otimes_{\mathbb{H}} A \otimes_{\mathbb{H}} S_{\mathbb{H}}^{l-1} A \stackrel{\text { id } \otimes_{\mathbb{H}} \xi_{A} \otimes_{\mathbb{H}} \text { id }}{\longrightarrow} \\
& S_{\mathbb{H}}^{k-1} A \otimes_{\mathbb{H}} A \otimes_{\mathbb{H}} Y \otimes_{\mathbb{H}} S_{\mathbb{H}}^{l-1} A \stackrel{\sigma_{\mathbb{H}}}{\longrightarrow} S_{\mathbb{H}}^{k+l-1} A \otimes_{\mathbb{H}} Y .
\end{aligned}
$$

Here $\iota$ is the inclusion, and $\sigma_{\mathbb{H}}$ the symmetrization operator of $\oint$ 1.1. For $k=0$ or $l=0$, let $\xi_{k, l}=0$. Define $\xi_{F A}: F^{A} \otimes_{\mathbb{H}} F^{A} \rightarrow F^{A} \otimes_{\mathbb{H}} Y$ to be the unique linear map such that the restriction of $\xi_{F^{A}}$ to $S_{\mathbb{H}}^{k} A \otimes_{\mathbb{H}} S_{\mathbb{H}}^{l} A$ is $\xi_{k, l}$, for all $k, l \geq 0$. Then $\xi_{F^{A}}$ is a well-defined AHI-morphism. A calculation following those in [7, §1.4] shows that $\xi_{F^{A}}$ satisfies Axioms P1 and P2 of $\S 2.2$. Thus, by Definition 2.2.2, $F^{A}$ is an HP-algebra. Moreover, $F^{A}$ is a filtered H-algebra with the natural filtration, and it is easy to show that Axiom PF holds. So $F^{A}$ is a filtered HP-algebra.

Combining Examples 4.3.1 and 4.3.4, we see that if $\mathfrak{g}$ is a Lie algebra, then $F^{A_{\mathfrak{g}}}$ is a filtered HP-algebra.

\subsection{Hyperkähler manifolds with symmetries}

Let $M$ be a hyperkähler manifold, and suppose $v$ is a Killing vector of the hyperkähler structure on $M$. A hyperkähler moment map for $v$ is a triple $\left(f_{1}, f_{2}, f_{3}\right)$ of smooth real functions on $M$ such that $\alpha=I_{1} d f_{1}=I_{2} d f_{2}=I_{3} d f_{3}$, where $\alpha$ is the 1 -form dual to $v$ under the metric $g$. Moment maps always exist if $b^{1}(M)=0$, and are unique up to additive constants.

More generally, let $M$ be a hyperkähler manifold, let $G$ be a Lie group with Lie algebra $\mathfrak{g}$, and suppose $\Phi: G \rightarrow \operatorname{Aut}(M)$ is a homomorphism from 
$G$ to the group of automorphisms of the hyperkähler structure on $M$. Let $\phi: \mathfrak{g} \rightarrow \operatorname{Vect}(M)$ be the induced map from $\mathfrak{g}$ to the Killing vectors. Then a hyperkähler moment map for the action $\Phi$ of $G$ is a triple $\left(f_{1}, f_{2}, f_{3}\right)$ of smooth functions from $M$ to $\mathfrak{g}^{*}$, such that for each $x \in \mathfrak{g},\left(x \cdot f_{1}, x \cdot f_{2}, x \cdot f_{3}\right)$ is a hyperkähler moment map for the vector field $\phi(x)$, and in addition, $\left(f_{1}, f_{2}, f_{3}\right)$ is equivariant under the action $\Phi$ of $G$ on $M$ and the coadjoint action of $G$ on $\mathfrak{g}^{*}$.

Moment maps are a familiar part of symplectic geometry, and hyperkähler moment maps were introduced by Hitchin et al. as part of a quotient construction for hyperkähler manifolds [12], [26, p. 118-122]. Hyperkähler moment maps will exist under quite mild conditions on $M$ and $G$, for instance if $b^{1}(M)=0$ and $G$ is compact. We shall use them to construct q-holomorphic functions on hyperkähler manifolds with symmetries.

Example 4.4.1 Let $M$ be a hyperkähler manifold and $v$ a nonzero Killing vector of the hyperkähler structure on $M$. Suppose $\left(f_{1}, f_{2}, f_{3}\right)$ is a hyperkähler moment map for $v$. We shall make q-holomorphic functions on $M$ out of the real functions $f_{j}$. Let $q_{1}, q_{2}, q_{3} \in \mathbb{H}$, and consider the $\mathbb{H}$ valued function $y=q_{1} f_{1}+q_{2} f_{2}+q_{3} f_{3}$ on $M$. By construction the $f_{j}$ satisfy $I_{1} d f_{1}=I_{2} d f_{2}=I_{3} d f_{3}$. Using this equation, the fact that $v$ is nonzero, and the definition of q-holomorphic in $\S 3.1$, it is easy to show that $y$ is q-holomorphic if and only if $q_{1} i_{1}+q_{2} i_{2}+q_{3} i_{3}=0$. Thus we have constructed an AHI-module $Y \cong\left\{\left(q_{1}, q_{2}, q_{3}\right) \in \mathbb{H}^{3}: q_{1} i_{1}+q_{2} i_{2}+q_{3} i_{3}=0\right\}$ of q-holomorphic functions on $M$. It is isomorphic to the AHI-module $Y$ of Definition 2.2.1.

Example 4.4.2 Now let $M$ be a hyperkähler manifold, $G$ a Lie group, $\Phi: G \rightarrow \operatorname{Aut}(M)$ an action of $G$ on $M$ preserving the hyperkähler structure, and $\phi: \mathfrak{g} \rightarrow \operatorname{Vect}(M)$ the induced map. Suppose $\phi$ is injective, and that $\left(f_{1}, f_{2}, f_{3}\right)$ is a hyperkähler moment map for $\Phi$. By Example 4.4.1, each nonzero $x \in \mathfrak{g}$ gives us an AHI-module $Y$ of q-holomorphic functions on $M$. Clearly, these fit together to form a canonical AHH-module $\mathfrak{g} \otimes Y$ of q-holomorphic functions on $M$.

We have already met $\mathfrak{g} \otimes Y=A_{\mathfrak{g}}$ as an HL-algebra in Example 4.3.1, where we defined a Poisson bracket $\xi_{A_{\mathfrak{g}}}$ on it. In the context of this example, $\mathfrak{g} \otimes Y$ is an AHI-submodule of the HP-algebra $A_{M}$ of q-holomorphic functions on $M$, which derives its own Poisson bracket $\xi_{A_{M}}$ from the hyperkähler structure of $M$. A computation shows that $\mathfrak{g} \otimes Y$ is closed under $\xi_{A_{M}}$, and that $\xi_{A_{M}}=\xi_{A_{\mathfrak{g}}}$. 
Example 4.4.2 shows that the HL-algebras of Example 4.3.1 are related to the HP-algebras of hyperkähler manifolds with symmetry groups. In the following lemma we extend this to the associated HP-algebras defined by Example 4.3.4.

Lemma 4.4.3 Let $M$ be a hyperkähler manifold, and $A_{M}$ the HP-algebra of $q$-holomorphic functions on $M$. Let $G$ be a Lie group, $\Phi: G \rightarrow \operatorname{Aut}(M)$ be an action of $G$ on $M$ preserving the hyperkähler structure, and $\phi: \mathfrak{g} \rightarrow \operatorname{Vect}(M)$ be the induced map. Suppose $\left(f_{1}, f_{2}, f_{3}\right)$ is a hyperkähler moment map for $\Phi$. Then there is a canonical HP-algebra morphism $\Phi_{*}: F^{A_{\mathfrak{g}}} \rightarrow A_{M}$, where $F^{A_{\mathfrak{g}}}$ is defined by Examples 4.3.1 and 4.3.4.

Proof. In Example 4.4 .2 we constructed an AHI-submodule $A_{\mathfrak{g}}=\mathfrak{g} \otimes Y$ of $A_{M}$. By Lemma 2.4.4, there is a unique H-algebra morphism $\Phi_{*}=\phi_{A_{\mathfrak{g}}}$ : $F^{A_{\mathfrak{g}}} \rightarrow A_{M}$. To complete the proof we must show that $\Phi_{*}$ is an HP-algebra morphism, where the HP-algebra structure on $F^{A_{\mathfrak{g}}}$ is defined by Example 4.3.4, so we must show that $\Phi_{*}$ identifies the Poisson brackets on $F^{A_{\mathfrak{g}}}$ and $A_{M}$.

In Example 4.3.4 we remarked that $\xi_{A_{M}}=\xi_{A_{\mathfrak{g}}}$ on $A_{\mathfrak{g}}$. Thus $\Phi_{*}$ identifies the Poisson brackets on this subspace. Because $\Phi_{*}$ is an $\mathrm{H}$-algebra morphism and $A_{\mathfrak{g}}$ generates $F^{A_{\mathfrak{g}}}$, we can deduce from Axiom P2 that if $\xi_{A_{\mathfrak{g}}}$ and the pullback of $\xi_{A_{M}}$ agree on $A_{\mathfrak{g}}$, they must agree on the whole of $F^{A_{\mathfrak{g}}}$. Thus $\Phi_{*}$ identifies the Poisson brackets of $F^{A_{\mathfrak{g}}}$ and $A_{M}$, and $\Phi_{*}$ is an HP-algebra morphism.

In the situation of the lemma, $\Phi_{*}\left(F^{A_{\mathfrak{g}}}\right)$ is an HP-algebra containing information about $M$ and $G$. This suggests that to understand hyperkähler manifolds with symmetries better, it may be helpful to study HP-algebras that are images of $F^{A_{\mathfrak{g}}}$. The next two examples construct such images.

Example 4.4.4 Let $G$ be a Lie group with Lie algebra $\mathfrak{g}$. Then $A_{\mathfrak{g}}=\mathfrak{g} \otimes Y$ and $Y^{\dagger}=\mathbb{I}$ by definition, so $A_{\mathfrak{g}}^{\dagger}=\mathfrak{g}^{*} \otimes \mathbb{I}$. As in the proof of Lemma 3.4.2, each element $f$ of $F^{A_{\mathfrak{g}}}$ induces an $\mathbb{H}$-valued polynomial $\psi_{f}$ on $A_{\mathfrak{g}}^{\dagger}=\mathfrak{g}^{*} \otimes \mathbb{I}$. Now $G$ acts on $\mathfrak{g}^{*}$ by the coadjoint action, so $G$ acts on $\mathfrak{g}^{*} \otimes \mathbb{I}$, with trivial action on $\mathbb{I}$. Let $\Omega \subset \mathfrak{g}^{*} \otimes \mathbb{I}$ be an orbit of $G$, that is contained in no proper vector subspace of $\mathfrak{g}^{*} \otimes \mathbb{I}$. Define an AHH-submodule $I^{\Omega}$ in $F^{A_{\mathfrak{g}}}$ by

$$
I^{\Omega}=\left\{f \in F^{A_{\mathfrak{g}}}: \psi_{f} \equiv 0 \text { on } \Omega\right\} .
$$

Clearly, $\mu_{F^{A_{\mathfrak{g}}}}\left(I^{\Omega} \otimes_{\mathbb{H}} F^{A_{\mathfrak{g}}}\right) \subset I^{\Omega}$, so $I^{\Omega}$ is an ideal. 
Define a subset $M^{\Omega} \subset \mathfrak{g}^{*} \otimes \mathbb{I}$ by

$$
M^{\Omega}=\left\{x \in \mathfrak{g}^{*} \otimes \mathbb{I}: \psi_{f}(x)=0 \text { for all } f \in I^{\Omega}\right\} .
$$

Then $M^{\Omega}$ is an affine real algebraic variety in $\mathfrak{g}^{*} \otimes \mathbb{I}$, as in Lemma 3.4.2. Also, $\Omega \subset M^{\Omega}$ by (21), and $M^{\Omega}$ is invariant under the action of $G$ on $\mathfrak{g}^{*} \otimes \mathbb{I}$. Define $A^{\Omega}=\left\{\left.\psi_{f}\right|_{M^{\Omega}}: f \in F^{A_{\mathfrak{g}}}\right\}$. Thus $A^{\Omega}$ is a vector space of $\mathbb{H}$-valued functions on $M^{\Omega}$.

Now $I^{\Omega}, F^{A_{\mathfrak{g}}}$ and $A^{\Omega}$ fit into a natural, AH-exact sequence

$$
0 \rightarrow I^{\Omega} \stackrel{\iota}{\longrightarrow} F^{A_{\mathfrak{g}}} \stackrel{\rho}{\longrightarrow} A^{\Omega} \rightarrow 0
$$

where $\iota$ is the inclusion map, and $\rho$ is the restriction map from $\mathfrak{g}^{*} \otimes \mathbb{I}$ to $M^{\Omega}$. Since $I^{\Omega}$ is an ideal, intuitively $A^{\Omega}$ should be an $\mathrm{H}$-algebra.

In $\$ 2.4$ we saw that the best sort of ideal is a stable filtered ideal. Therefore, let us assume that $I^{\Omega}$ is a stable filtered ideal. Then Lemma 2.4 .2 shows that $A^{\Omega}$ is an SFH-algebra, and $\rho$ a filtered H-algebra morphism. Also, because we suppose that $\Omega$ is not contained in any proper subspace of $\mathfrak{g}^{*} \otimes \mathbb{I}$, and $\Omega \subset M^{\Omega}$, we see that $\left.\rho\right|_{\mathfrak{g} \otimes Y}$ is injective, and $\mathfrak{g} \otimes Y$ is an AH-submodule of $A^{\Omega}$ that generates $A^{\Omega}$. It can be shown that $M_{A^{\Omega}, \mathfrak{g} \otimes Y}=M^{\Omega}$. Observe that $A^{\Omega}$ is an FGH-algebra in the sense of $\$ 2.4$.

Example 4.4.5 In the situation of the previous example, we shall show that $A^{\Omega}$ is an HP-algebra. The linear map $\xi_{F^{A_{\mathfrak{g}}}}: F^{A_{\mathfrak{g}}} \otimes_{\mathbb{H}} A_{\mathfrak{g}} \rightarrow F^{A_{\mathfrak{g}}} \otimes_{\mathbb{H}} Y$ is easy to understand, because $A_{\mathfrak{g}}=\mathfrak{g} \otimes Y$, so we may write the map as $\xi_{F^{A_{\mathfrak{g}}}}: \mathfrak{g} \otimes\left(F^{A_{\mathfrak{g}}} \otimes_{\mathbb{H}} Y\right) \rightarrow F^{A_{\mathfrak{g}}} \otimes_{\mathbb{H}} Y$, which just gives the Lie algebra action of $\mathfrak{g}$ on $F^{A_{\mathfrak{g}}}$. Since $I^{\Omega}$ is $G$-invariant, it follows that $\xi_{F^{A_{\mathfrak{g}}}}$ maps $I^{\Omega} \otimes_{\mathbb{H}} A_{\mathfrak{g}}$ to $I^{\Omega} \otimes_{\mathbb{H}} Y$. But $A_{\mathfrak{g}}$ generates $F^{A_{\mathfrak{g}}}$, and so using Axiom P2 and the fact that $\mu_{F^{A_{\mathfrak{g}}}}\left(I^{\Omega} \otimes_{\mathbb{H}} F^{A_{\mathfrak{g}}}\right) \subset I^{\Omega}$, it follows that $\xi_{F^{A_{\mathfrak{g}}}}\left(I^{\Omega} \otimes_{\mathbb{H}} F^{A_{\mathfrak{g}}}\right) \subset I^{\Omega} \otimes_{\mathbb{H}} Y$.

Using the assumption in Example 4.4.4, this inclusion is just what is needed to prove that the Poisson bracket $\xi_{F^{A}}$ can be pushed down to $A^{\Omega}$ using $\rho$, inducing a Poisson bracket $\xi_{A^{\Omega}}$ on $A^{\Omega}$, so that $A^{\Omega}$ is an HP-algebra. As $\rho$ is a filtered H-algebra morphism, $A^{\Omega}$ is a filtered HP-algebra, and $\rho$ a filtered HP-algebra morphism.

Examples 4.4.4 and 4.4.5 construct a large family of HP-algebras $A^{\Omega}$ associated to Lie groups. As in $\$ 3.4$, we can try to use $A^{\Omega}$ to construct a hyperkähler structure on $M^{\Omega}$. This suggests that associated to each Lie group $G$, there is a natural family of hyperkähler manifolds. Now Kronheimer 
[19, 20], Biquard [8] and Kovalev [16] have also constructed hyperkähler manifolds associated to Lie groups, from a completely different point of view.

Let $G$ be a compact Lie group with Lie algebra $\mathfrak{g}$, and let the complexification of $G$ be $G^{c}$ with Lie algebra $\mathfrak{g}^{c}$. Kronheimer found that certain moduli spaces of singular $G$-instantons on $\mathbb{R}^{4}$ are hyperkähler manifolds. These moduli spaces can be identified with coadjoint orbits of $G^{c}$ in $\left(\mathfrak{g}^{c}\right)^{*}$, and have hyperkähler metrics invariant under $G$. Kronheimer's construction worked only for certain special coadjoint orbits, and more general cases were handled by Biquard and Kovalev.

Although these metrics look very algebraic, their construction is in fact analytic, and the algebraic description of these metrics is not well understood. I propose that Examples 1.4 .4 and 1.4 .5 provide this algebraic description. This was proved in [14, §11-12] for Kronheimer's metrics [19, 20], which are the simplest case, but I have not yet proved it for the metrics of Biquard and Kovalev. Here is a conjecture about this.

Conjecture 4.4.6 We conjecture the following relations between Examples 4.4 .4 and 1.4.5, and Kronheimer, Biquard and Kovalev's 'coadjoint orbit' metrics.

- The assumption made in Example 4.4 .4 always holds.

- Using the techniques of $\S 3.4$, the HP-algebra $A^{\Omega}$ determines a hyperkähler structure on a dense open set of $M^{\Omega}$.

- These hyperkähler structures on subsets of $M^{\Omega}$ include all those of [19], [20], [8] and [16] as special cases. However, generically the structures on $M^{\Omega}$ are new, and do not coincide with those of Kronheimer, Biquard and Kovalev.

- For some $\Omega, M^{\Omega}$ is a cone in $\mathfrak{g}^{*} \otimes \mathbb{I}$, and $A^{\Omega}$ is an SGH-algebra. Then $M^{\Omega}$ is a hyperkähler cone, as in $\$ 3.5$. These are the nilpotent orbits of [20].

- For each $\Omega$ there is an $\tilde{\Omega}$, such that $M^{\tilde{\Omega}}$ is a 'nilpotent orbit', and the associated graded $\mathrm{H}$-algebra of $A^{\Omega}$ is $A^{\tilde{\Omega}}$. Then the (singular) hyperkähler manifold $M^{\Omega}$ has an $\mathrm{AC}$ end, as in Definition 3.5.5, with asymptotic cone $M^{\tilde{\Omega}}$.

Further study of these spaces from the HP-algebra point of view will probably lead to a much clearer understanding of the algebra and geometry 
underlying Kronheimer's metrics. We will look at an example in detail in the next section.

\subsection{The Eguchi-Hanson space}

The Eguchi-Hanson space $M$ is a noncompact hyperkähler manifold of dimension 4, with a metric written down by Eguchi and Hanson [9]. It is of interest to us as an example for two reasons. Firstly, it is the simplest interesting example of a 'coadjoint orbit' metric, as it fits into the theory of $\$ 4.4$ with $G=S U(2)$ or $S O(3)$. Secondly, it is the simplest hyperkähler asymptotically locally Euclidean or ALE space. An ALE space is an AC hyperkähler manifold of dimension 4 , with asymptotic cone $\mathbb{H} / \Gamma$, for some finite subgroup $\Gamma \subset S U(2)$.

The ALE spaces for cyclic $\Gamma$ were described explicitly by Gibbons and Hawking [10] and Hitchin [11], and a complete construction and classification of ALE spaces was given by Kronheimer [17], [18]. The Eguchi-Hanson space has asymptotic cone $\mathbb{H} /\{ \pm 1\}$, and as a manifold it is diffeomorphic to the total space of $T^{*} \mathbb{C} \mathbb{P}^{1}$. In this section we will treat the Eguchi-Hanson space from our H-algebra point of view. It gives us our first explicit example of the theories of $\$ 3.5$ and $\S 4.4$. On the way, we will determine the deformations of the H-algebra of the Eguchi-Hanson space. The result and its implications are quite surprising.

Let $M$ be the Eguchi-Hanson space. Then the hyperkähler cone of $M$, in the sense of $\delta 3.5$, is $\mathbb{H} /\{ \pm 1\}$. Our first step is to find the SGH-algebra $B$ of q-holomorphic functions on $\mathbb{H} /\{ \pm 1\}$ with polynomial growth.

Example 4.5.1 By Example 4.1.3, the graded H-algebra of q-holomorphic functions on $\mathbb{H}$ is $F^{U}$, where $U$ is defined in the example. Define $\sigma: \mathbb{H} \rightarrow \mathbb{H}$ by $\sigma(q)=-q$. Then $\sigma$ acts on $F^{U}$, and as the functions in $U$ are linear, $\sigma$ acts as -1 on $U$. Therefore $\sigma$ acts as $(-1)^{j}$ on $S_{\mathbb{H}}^{j} U$. Now the SGH-algebra $B$ of q-holomorphic functions on $\mathbb{H} /\{ \pm 1\}$ is just the $\sigma$-invariant part of $F^{U}$. Therefore $B=\bigoplus_{j=0}^{\infty} S_{\mathbb{H}}^{2 j} U$. For compatibility with $\S 3.5$ we adopt the grading $B^{2 j}=S_{\mathbb{H}}^{2 j} U$ and $B^{2 j+1}=\{0\}$, and the corresponding filtration.

A calculation using Proposition 1.2 .8 and the definitions of $Y$ and $U$ in $\S \S 2.2$ and 4.1 shows that $\operatorname{dim}\left(S_{\mathbb{H}}^{j} Y\right)=4 k, \operatorname{dim}\left(S_{\mathbb{H}}^{j} Y\right)^{\prime}=2 k+s$ with $k=j+1$ and $s=1$, and $\operatorname{dim}\left(S_{\mathbb{H}}^{2 j} U\right)=4 l, \operatorname{dim}\left(S_{\mathbb{H}}^{2 j} U\right)^{\prime}=2 l+t$ with $l=(2 j+1)(j+1)$ and $t=2 j+1$. Thus $l=(2 j+1) k$ and $t=(2 j+1) s$. In fact, by carefully investigating the geometry it can be shown that $S_{\mathbb{H}}^{2 j} U \cong \mathbb{R}^{2 j+1} \otimes S_{\mathbb{H}}^{j} Y$. 
Here is one way to see this. It is well-known that $S O(4)$ acts irreducibly on $\mathbb{R}^{4}$, but that $\mathbb{R}^{4} \otimes \mathbb{C}$ splits as $\mathbb{C}^{2} \otimes \mathbb{C}^{2}$. In the same way, $U$ is irreducible, but $U \otimes \mathbb{C}$ may be written as $\mathbb{C}^{2} \otimes W$, where $W$ is a complex AHI-module with $W \otimes_{\mathbb{H}} W=S_{\mathbb{H}}^{2} W \cong Y \otimes \mathbb{C}$ as complex AHI-modules. Thus we may write $B^{2 j} \cong \mathbb{R}^{2 j+1} \otimes S_{\mathbb{H}}^{j} Y$, where $\mathbb{R}^{2 j+1}$ is the real part of $\mathbb{C}^{2 j+1}=S^{j}\left(\mathbb{C}^{2}\right)$, and $B=\bigoplus_{j=0}^{\infty} \mathbb{R}^{2 j+1} \otimes S_{\mathbb{H}}^{j} Y$.

Next, we present $B$ as an FGH-algebra generated by $Q \subset B$, and determine the algebraic variety $M_{B, Q}$.

Example 4.5.2 To write $B$ as an FGH-algebra, the quotient of a free $\mathrm{H}$ algebra $F^{Q}$ by an ideal $I \subset F^{Q}$, one must choose a subspace $Q$ of $B$ that generates $B$. Define $Q=B^{2}=\mathbb{R}^{3} \otimes Y$, where $\mathbb{R}^{3}$ is equipped with a Euclidean metric and inner product. Then $\phi_{Q}: F^{Q} \rightarrow B$ is defined in Lemma 2.4.4, and we set $I=\operatorname{Ker} \phi_{Q}$. Since $B^{2 j}=\mathbb{R}^{2 j+1} \otimes S_{\mathbb{H}}^{j} Y$ and $S_{\mathbb{H}}^{j} Q=\mathbb{R}^{(j+1)(j+2) / 2} \otimes S_{\mathbb{H}}^{j} Y$, it can be shown that $\phi_{Q}: S_{\mathbb{H}}^{j} Q \rightarrow B^{2 j}$ is surjective for all $j \geq 0$, and has kernel $I^{2 j} \subset S_{\mathbb{H}}^{j} Q$ with $I^{2 j} \cong \mathbb{R}^{j(j-1) / 2} \otimes S_{\mathbb{H}}^{j} Y$.

Now $S_{\mathbb{H}}^{j} Y$ is stable by Proposition 1.2.8, as $Y$ is stable. Thus $I$ is a stable filtered ideal in the sense of Definition 2.4.1. Therefore $B$ is generated by $Q$, and $B$ is an FGH-algebra by Definition 2.4.5. Set $J=I^{4} \cong S_{\mathbb{H}}^{2} Y$. Another calculation shows that $I$ is generated by $J$ in the sense of Definition 2.4.1. Moreover, if $h$ is the Euclidean metric on $\mathbb{R}^{3}$, then the AHI-module $J \subset S_{\mathbb{H}}^{2} Q$ is $J=\langle h\rangle \otimes S_{\mathbb{H}}^{2} Y \subset S^{2} \mathbb{R}^{3} \otimes S_{\mathbb{H}}^{2} Y=S_{\mathbb{H}}^{2} Q$.

Thus $B$ is the quotient of the free SFH-algebra $F^{Q}$, where $Q=\mathbb{R}^{3} \otimes Y$, by the stable filtered ideal $I$ in $F^{Q}$, where $I$ is generated by the AHI-module $J=S_{\mathbb{H}}^{2} Y \subset S_{\mathbb{H}}^{2} Q$. This is an explicit description of $B$ as an FGH-algebra. To finish the example, we will find and describe the subset $M_{B, Q}$ of $Q^{\dagger}$, defined in $\oint$ 3.4. As $Q=\mathbb{R}^{3} \otimes Y, Q^{\dagger}=\left(\mathbb{R}^{3}\right)^{*} \otimes \mathbb{I}$, as $Y^{\dagger} \cong \mathbb{I}$. Identify $\left(\mathbb{R}^{3}\right)^{*}$ with $\mathbb{R}^{3}$ using the metric. Then a point $\gamma$ in $Q^{\dagger}$ may be written $\gamma=\Sigma_{k=1}^{3} v_{k} \otimes i_{k}$, where $v_{1}, v_{2}, v_{3}$ are vectors in $\mathbb{R}^{3}$.

The proof of Lemma 3.4.2 showed that each element $y$ of $F^{Q}$ defines an $\mathbb{H}$ valued polynomial $\psi_{y}$ on $Q^{\dagger}$. In our case, as $J$ generates $I, M_{B, Q}$ is the zeros of the polynomials $\psi_{y}$ for $y \in J$. Moreover, as $J \subset S_{\mathbb{H}}^{2} Q$, these polynomials are homogeneous quadratics on $Q^{\dagger}$. A computation using the definitions of $Y, Q$ and $J$ shows that $\gamma \in M_{B, Q}$ if and only if

$$
v_{1} \cdot v_{1}=v_{2} \cdot v_{2}=v_{3} \cdot v_{3}, \quad v_{1} \cdot v_{2}=0, \quad v_{2} \cdot v_{3}=0, \quad v_{3} \cdot v_{1}=0,
$$

where '.' is the inner product on $\mathbb{R}^{3}$. So $v_{1}, v_{2}, v_{3}$ must be orthogonal in $\mathbb{R}^{3}$, 
and of equal length.

Now we look at $M_{B, Q}$ more closely, and interpret it as a 'coadjoint orbit'.

Example 4.5.3 The previous example identified the algebraic variety $M_{B, Q}$ with the set of triples $\left(v_{1}, v_{2}, v_{3}\right)$ of vectors in $\mathbb{R}^{3}$ that are orthogonal and of equal length. For each $r>0$, define $S_{r,+}$ to be the set of orthogonal triples $\left(v_{1}, v_{2}, v_{3}\right)$ that form a positively oriented basis of $\mathbb{R}^{3}$, and for which $\left|v_{1}\right|=\left|v_{2}\right|=\left|v_{3}\right|=r$. Let $S_{r,-}$ be defined in the same way, but with negative orientation. Then $S_{r,+}$ and $S_{r,-}$ are subsets of $M_{B, Q}$.

Now $S O(3)$ acts on $\mathbb{R}^{3}$ preserving the Euclidean metric, and the action preserves the equations (24). Thus $M_{B, Q}$ decomposes into orbits of the $S O(3)$ - action. It is easy to show that the orbits are the single point $(0,0,0)$ and the sets $S_{r, \pm}$ for $r>0$. Moreover, $S O(3)$ acts freely on the set $S_{r, \pm}$, so that $S_{r, \pm} \cong S O(3) \cong \mathbb{R P}^{3}$. Therefore $M_{B, Q}$ is the disjoint union of $\{0\}$ and 2 copies of $(0, \infty) \times \mathbb{R P}^{3}$.

However, $\mathbb{H} /\{ \pm 1\}$ is the disjoint union of $\{0\}$ and 1 copy of $(0, \infty) \times \mathbb{R P}^{3}$. Therefore, $M_{B, Q}$ is actually the union of two distinct copies of $\mathbb{H} /\{ \pm 1\}$, which meet at 0 , and the map $\pi_{B, Q}: \mathbb{H} /\{ \pm 1\} \rightarrow M_{B, Q}$ is an isomorphism with one of these copies. This is something of a surprise. The 2 copies are not separate algebraic components of the variety $M_{B, Q}$, as the complexification of $M_{B, Q}$ in $Q^{\dagger} \otimes \mathbb{C}$ has only one component containing both copies.

Let us look at $B$ from the point of view of $\$ 4.4$. Above we gave an action of $S O(3)$ on $M_{B, Q}$. This action induces an isomorphism between the $\mathbb{R}^{3}$ in the equation $Q=\mathbb{R}^{3} \otimes Y$, with the Lie algebra $\mathfrak{s o}(3)$ of $S O(3)$. Thus $Q \cong A_{\mathfrak{s o}(3)}$, where $A_{\mathfrak{s o}(3)}$ is defined in Example 4.3.1. So $B$ is the quotient of $F^{A_{\mathfrak{s o}(3)}}$ by a stable filtered ideal. Therefore, $B$ fits into framework of Example 4.4.4.

In fact, $S_{r \pm}$ are orbits in $\mathfrak{s o}(3)^{*} \otimes \mathbb{I}$, and if we put $\Omega=S_{r,+}$ or $S_{r,-}$ for any $r>0$, then calculation shows that $A^{\Omega}=B$, so that $M^{\Omega}=M_{B, Q}$. This is the simplest example of an HP-algebra of this form, and it is one of the 'nilpotent orbits' mentioned in Conjecture 4.4.6.

Our goal, remember, is to find the filtered H-algebra $P$ of q-holomorphic functions of polynomial growth on the Eguchi-Hanson space $M$. From the definition [9] of the Eguchi-Hanson metric one finds that $M$ is AC to order 4 , with asymptotic cone $\mathbb{H} /\{ \pm 1\}$. Therefore, Theorem 3.5.6 applies to show that $P$ is an SFH-algebra and $B$ and $P$ are isomorphic to order 4 , in the sense of Definition 2.3.5. In the next example we will determine all such SFH-algebras. 
Example 4.5.4 Suppose $P$ is an SFH-algebra isomorphic to order 4 with the $\mathrm{H}$-algebra $B$ of the previous examples. As $B_{-2}, P_{-2}$ are zero, $\phi_{2}: B_{2} \rightarrow P_{2}$ is an $\mathrm{A} \mathbb{H}$-isomorphism. Thus $P_{2} \cong \mathbb{H} \oplus Q$. Since $Q$ generates $B$, Proposition 2.4.6 shows that $P_{2}$ generates $P$. But the $\mathbb{H}$ in $P_{2}$ is the multiples of 1 , so it does not generate anything. Therefore $P$ is generated by $Q$, and $P$ is the quotient of $F^{Q}$ by a stable filtered ideal $I^{P}$.

Define $J^{P}=I_{4}^{P}$, so that $J^{P}$ is an AH-submodule of $\mathbb{H} \oplus Q \oplus S_{\mathbb{H}}^{2} Q$. By a similar argument to Proposition 2.4.6, we find that because $J$ generates $I$, $J^{P}$ generates $I^{P}$. Thus $P$ is determined by the AH-submodule $J^{P}$. Using the maps $\phi_{2}, \phi_{4}$ to compare $I^{P}$ and $I$, we see that $I_{2}^{P}=\{0\}$, that $J^{P} \subset \mathbb{H} \oplus J$. Let $\pi_{1}, \pi_{2}$ be the projections from $\mathbb{H} \oplus J$ to the $\mathbb{H}$ and $J$. Then $\pi_{2}: J^{P} \rightarrow J$ is an AHI-isomorphism. Let $\iota: J \rightarrow J^{P}$ be its inverse, so that $\iota: J \rightarrow \mathbb{H} \oplus J$ is an AH-morphism with $J^{P}=\iota(J)$.

As $\pi_{2} \circ \iota$ is the identity on $J, J^{P}$ is determined by $\pi_{1} \circ \iota$, i.e. by an AHmorphism from $J$ to $\mathbb{H}$. Let $\lambda \in J^{\dagger}$. Then $\lambda \in J^{\times}$, so $\lambda: J \rightarrow \mathbb{H}$ is an $\mathbb{H}$-linear map. By definition of $J^{\dagger}, \lambda$ maps $J^{\prime}$ to $\mathbb{I}$ which is $\mathbb{H}^{\prime}$. Thus $\lambda$ is an AH-morphism from $J$ to $\mathbb{H}$, and it is easy to see that all such AH-morphisms are elements of $J^{\dagger}$.

Therefore, we have shown that all SFH-algebras $P$ isomorphic with $B$ to order 4 (in the sense of Definition 2.3.5) are constructed in the following manner. We are given $Q=\mathbb{R}^{3} \otimes Y$, and a fixed AHI-module $J \subset S_{\mathbb{H}}^{2} Q$, that has $J \cong S_{\mathbb{H}}^{2} Y$, so that $J^{\dagger} \cong \mathbb{R}^{5}$. Choose an element $\lambda \in J^{\dagger}$. Then $\lambda: J \rightarrow \mathbb{H}$ is an AH-morphism. Define $J^{\lambda}$ to be the image of the AHImorphism $\lambda \oplus$ id $: J \rightarrow \mathbb{H} \oplus J$.

As $\mathbb{H}=S_{\mathbb{H}}^{0} Q$ and $J \subset S_{\mathbb{H}}^{2} Q$, we may regard $\mathbb{H} \oplus J$ as an AHH-submodule

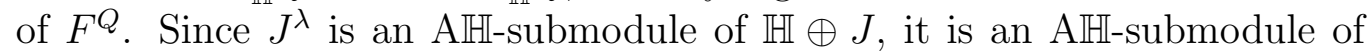
$F^{Q}$. Define $I^{\lambda}$ by $I^{\lambda}=\mu_{F^{Q}}\left(J^{\lambda} \otimes_{\mathbb{H}} F^{Q}\right)$. Then $I^{\lambda}$ is a stable filtered ideal of $F^{Q}$. Define $P^{\lambda}$ to be the quotient of $F^{Q}$ by $I^{\lambda}$, following Lemma 2.4.2. By Definition 2.4.5, $P^{\lambda}$ is an FGH-algebra.

Next we identify the variety $M_{P^{\lambda}, Q}$.

Example 4.5.5 Let $\lambda \in J^{\dagger}$ be given. Then the previous example defines an FGH-algebra $P^{\lambda}$, generated by $Q \subset P^{\lambda}$. We shall describe the real algebraic variety $M_{P^{\lambda}, Q} \subset Q^{\dagger}$. Our treatment follows Example 4.5.2 closely, and uses the same notation. Now $J^{\lambda}$ may be interpreted as a vector space of $\mathbb{H}$-valued polynomials on $Q^{\dagger}$, and $M_{P^{\lambda}, Q}$ is the zeros of these polynomials.

There is a natural identification between $\left(S_{\mathbb{H}}^{2} Y\right)^{\dagger}$ and $S_{0}^{2} \mathbb{R}^{3}$, which is the space of trace-free $3 \times 3$ symmetric matrices. Using this identification we 
may write $\lambda$ in coordinates as $\lambda=\left(a_{k l}\right)$, where $\left(a_{k l}\right)$ is a $3 \times 3$ matrix with $a_{j k}=a_{k j}$ and $\Sigma_{j} a_{j j}=0$. Using the vectors $v_{1}, v_{2}, v_{3}$ as coordinates on $Q^{\dagger}$ as before, the equations defining $M_{P^{\lambda}, Q}$ turn out to be

$$
\begin{aligned}
& v_{1} \cdot v_{1}-a_{11}=v_{2} \cdot v_{2}-a_{22}=v_{3} \cdot v_{3}-a_{33}, \\
& v_{1} \cdot v_{2}=a_{12}, \quad v_{2} \cdot v_{3}=a_{23}, \quad v_{3} \cdot v_{1}=a_{31} .
\end{aligned}
$$

These are 5 real equations, because $\left(J^{\lambda}\right)^{\dagger} \cong \mathbb{R}^{5}$. When $\lambda=0, a_{k l}=0$ and we recover the equations (24).

As in Example 4.5.3, the equations (25) are invariant under the action of $S O(3)$ on $\mathbb{R}^{3}$. Thus $M_{P^{\lambda}, Q}$ is invariant under the $S O(3)$ - action, and is a union of $S O(3)$ orbits. Choose any point $m=\left(v_{1}, v_{2}, v_{3}\right)$ in $M_{P^{\lambda}, Q}$ such that $v_{1}, v_{2}, v_{3}$ are linearly independent (this is true for a generic point), and let $\Omega$ be the orbit of $m$. Then it can be shown that $A^{\Omega}=P^{\lambda}$ and $M^{\Omega}=M_{P^{\lambda}, Q}$, as in Example 4.5.3. Thus $P^{\lambda}$ is one of the algebras $A^{\Omega}$ of Example 4.4.4, with Lie group $G=S O(3)$. Therefore, by Example 4.4.5, $P^{\lambda}$ is a filtered HP-algebra.

Finally, we interpret $M_{P^{\lambda}, Q}$, and describe its hyperkähler structure.

Example 4.5.6 In Example 4.5.5, the element $\lambda \in J^{\dagger}$ determines a matrix $\left(a_{j k}\right)$. Now $S O(3)$ acts on $\mathbb{I}$, inducing automorphisms of $\mathbb{H}$. But automorphisms of $\mathbb{H}$ act on HP-algebras in an natural way. Thus $S O(3)$ acts on the category of HP-algebras. This action of $S O(3)$ on the HP-algebras $P^{\lambda}$ turns out to be conjugation of the matrix $\left(a_{j k}\right)$ by elements of $S O(3)$. So, up to automorphisms of $\mathbb{H}$, we may suppose that $P^{\lambda}$ is defined by a matrix $\left(a_{j k}\right)$ that is diagonal, and for which $a_{11} \geq a_{22} \geq a_{33}$.

Let the matrix $\left(a_{j k}\right)$ be of this form. Then the equations (25) can be rewritten

$$
v_{1} \cdot v_{1}-a=v_{2} \cdot v_{2}-b=v_{3} \cdot v_{3}, \quad v_{1} \cdot v_{2}=0, \quad v_{2} \cdot v_{3}=0, \quad v_{3} \cdot v_{1}=0,
$$

where $a, b$ are constants with $a \geq b \geq 0$. We shall consider the following three cases separately. Case 1 is $a=b=0$, case 2 is $a>0, b=0$, and case 3 is $a \geq b>0$. In case 1 , we have $\lambda=0$, so $P^{\lambda}=B$ and $M_{P^{\lambda}, Q}$ is 2 copies of $\mathbb{H} /\{ \pm 1\}$ meeting at 0 , as in Example 4.5.3.

In case 2 , there is one special orbit of $S O(3)$, the orbit $\left\{\left(v_{1}, 0,0\right): v_{1} \cdot v_{1}=\right.$ a\}. Clearly this is a 2 -sphere $\mathcal{S}^{2}$. It is easy to see that $M_{P^{\lambda}, Q}$ is the union 
of $\mathcal{S}^{2}$ with 2 copies of $(0, \infty) \times \mathbb{R P}^{3}$. Also, $M_{P^{\lambda}, Q}$ is singular at $\mathcal{S}^{2}$ but nonsingular elsewhere. A more careful investigation shows that $M_{P^{\lambda}, Q}$ is actually the union of 2 nonsingular, embedded submanifolds $M_{1}, M_{2}$ of $Q^{\dagger}$, that meet in a common $\mathcal{S}^{2}$. In fact, both $M_{1}$ and $M_{2}$ are the Eguchi-Hanson space. This case is the HP-algebra of the Eguchi-Hanson space $M$, and the map $\pi_{P^{\lambda}, Q}: M \rightarrow M_{P^{\lambda}, Q}$ is a diffeomorphism from $M$ to $M_{1}$, say.

Thus, we have found the HP-algebra $P^{\lambda}$ of q-holomorphic functions of polynomial growth on the Eguchi-Hanson space $M$. When we attempt to reconstruct $M$ from $P^{\lambda}$ we find that $M_{P^{\lambda}, Q}$ contains not one but two distinct copies of $M$, that intersect in an $\mathcal{S}^{2}$.

In case $3, S O(3)$ acts freely on $M_{P^{\lambda}, Q}$, and $M_{P^{\lambda}, Q}$ is a nonsingular submanifold of $Q^{\dagger}$ diffeomorphic to $\mathbb{R} \times \mathbb{R}^{3}$. This 4-manifold has two ends, each modelled on that of $\mathbb{H} /\{ \pm 1\}$. In cases 1 and 2 , we saw that $M_{P^{\lambda}, Q}$ was the singular union of 2 copies of $\mathbb{H} /\{ \pm 1\}$ or the Eguchi-Hanson space; in case 3 this singular union is resolved into one nonsingular 4-manifold.

Let us apply the programme of $\$ 3.4$, to construct a hypercomplex structure on $M_{P^{\lambda}, Q}$. This can be done, and as $Q$ is stable, Proposition 3.4.5 shows that the hypercomplex structure is determined by $P^{\lambda}$ wherever it exists. At first sight, therefore, it seems that $M_{P^{\lambda}, Q}$ carries a nonsingular hypercomplex structure with two ends, both asymptotic to $\mathbb{H} /\{ \pm 1\}$. However, explicit calculation reveals that although $M_{P^{\lambda}, Q}$ is nonsingular, its hypercomplex structure has a singularity on the hypersurface $v_{3}=0$ in $M_{P^{\lambda}, Q}$, which is diffeomorphic to $\mathbb{R} \mathbb{P}^{3}$.

What is the nature of this hypersurface singularity? Consider the involution $\left(v_{1}, v_{2}, v_{3}\right) \mapsto\left(-v_{1},-v_{2},-v_{3}\right)$ of $M_{P^{\lambda}, Q}$. This preserves the hypercomplex structure, but it is orientation-reversing on $M_{P^{\lambda}, Q}$. It also preserves the hypersurface $v_{3}=0$. Now a hypercomplex structure has its own natural orientation. Therefore, the hypercomplex structure changes orientation over its singular hypersurface $v_{3}=0$.

This singular hypercomplex manifold has a remarkable property. Each element of $P^{\lambda}$ is a q-holomorphic function on $M_{P^{\lambda}, Q}$, which is smooth, as $M_{P^{\lambda}, Q}$ is a submanifold of $Q^{\dagger}$. Thus, the hypercomplex manifold has a full complement of q-holomorphic functions that extend smoothly over the singularity. I shall christen singularities with this property invisible singularities, because you cannot see them using q-holomorphic functions. I think this phenomenon is worth further study.

As $P^{\lambda}$ is an HP-algebra, $M_{P^{\lambda}, Q}$ is actually hyperkähler. In Example 4.5 .5 we showed that this hyperkähler structure is $S O(3)$-invariant. Now in [6], 
Belinskii et al. explicitly determine all hyperkähler metrics with an $S O(3)$ action of this form, by solving an ODE. Thus, the metric on $M_{P^{\lambda}, Q}$ is given in [6]. Belinskii et al. show that the singularity is a curvature singularity that is, the Riemann curvature becomes infinite upon it.

The metrics can also be seen from the twistor point of view. In 11], Hitchin constructs the twistor spaces of some ALE spaces, including the Eguchi-Hanson space. He uses a polynomial $z^{k}+a_{1} z^{k-1}+\cdots+a_{k}$, introduced on [11, p. 467]. On p. 468 he assumes this polynomial has a certain sort of factorization, to avoid singularities. If this assumption is dropped, then in the case $k=2$, Hitchin's construction yields the twistor spaces of our singular hyperkähler manifolds $M_{P^{\lambda}, Q}$.

\subsection{Self-dual vector bundles and H-algebra modules}

First we define q-holomorphic sections of a vector bundle.

Definition 4.6.1 Let $M$ be a hypercomplex manifold. Let $E$ be a vector bundle over $M$, and $\nabla_{E}$ a connection on $E$. Define an operator $D_{E}: \mathbb{H} \otimes$ $C^{\infty}(E) \rightarrow C^{\infty}\left(E \otimes T^{*} M\right)$ by

$$
\begin{aligned}
& D_{E}\left(1 \otimes e_{0}+i_{1} \otimes e_{1}+i_{2} \otimes e_{2}+i_{3} \otimes e_{3}\right)= \\
& \nabla_{E} e_{0}+I_{1}\left(\nabla_{E} e_{1}\right)+I_{2}\left(\nabla_{E} e_{2}\right)+I_{3}\left(\nabla_{E} e_{3}\right),
\end{aligned}
$$

where $I_{1}, I_{2}, I_{3}$ act on the $T^{*} M$ factor of $E \otimes T^{*} M$, and $e_{0}, \ldots, e_{3} \in C^{\infty}(E)$. We call an element $e$ of $\mathbb{H} \otimes C^{\infty}(E)$ a q-holomorphic section if $D_{E}(e)=0$.

Define $Q_{M, E}$ to be the vector space of q-holomorphic sections in $\mathbb{H} \otimes$ $C^{\infty}(E)$. Then $Q_{M, E}$ is closed under the $\mathbb{H}$-action $p \cdot(q \otimes e)=(p q) \cdot e$ on $\mathbb{H} \otimes C^{\infty}(E)$, so $Q_{M, E}$ is an $\mathbb{H}$-module. Define a real vector subspace $Q_{M, E}^{\prime}$ by $Q_{M, E}^{\prime}=Q_{M, E} \cap \mathbb{I} \otimes C^{\infty}(E)$. As in Definition 3.2.1, one can show that $Q_{M, E}$ is an AHI-module.

The point of this definition is the following theorem. The proof follows that of Theorem 3.2.5 in $\$ 3.2$ very closely, so we leave it as an exercise.

Theorem 4.6.2 Let $M$ be a hypercomplex manifold, and $A_{M}$ the H-algebra of q-holomorphic functions on $M$. Let $E$ be a vector bundle over $M$, with connection $\nabla_{E}$. Then the AHI-module $Q_{M, E}$ of Definition 4.6 .1 is a module over the H-algebra $A_{M}$, in a natural way. 
Recall that modules over H-algebras were defined in Definition 2.1.1. To see the link between Theorems 3.2 .5 and 4.6 .2 , put $E=\mathbb{R}$ with the flat connection. Then a q-holomorphic section of $E$ is a q-holomorphic function, so $Q_{M, E}=A_{M}$, and Theorem 4.6.2 states that $A_{M}$ is a module over itself. But this follows trivially from Theorem 3.2.5.

Now the equation $D_{E}(e)=0$ is in general overdetermined, and for a generic connection $\nabla_{E}$ we find that $Q_{M, E}=\{0\}$ for $\operatorname{dim} M>4$, and $Q_{M, E}^{\prime}=\{0\}$ for $\operatorname{dim} M=4$. In these cases, Theorem 1.6.2 is trivial. To make the situation interesting, we need $\nabla_{E}$ to satisfy a curvature condition (integrability condition), ensuring that $D_{E}(e)=0$ has many solutions locally. We will give this condition. The following proposition is a collection of results from [24, §2].

Proposition 4.6.3 Let $M$ be a hypercomplex manifold of dimension $4 n$. Then $I_{1}, I_{2}, I_{3}$ act as maps $T^{*} M \rightarrow T^{*} M$, so we may consider the map $\delta: \Lambda^{2} T^{*} M \rightarrow \Lambda^{2} T^{*} M$ defined by $\delta=I_{1} \otimes I_{1}+I_{2} \otimes I_{2}+I_{3} \otimes I_{3}$. Then $\delta^{2}=2 \delta+3$, so the eigenvalues of $\delta$ are 3 and -1 . This induces a splitting $\Lambda^{2} T^{*} M=\Lambda_{+} \oplus \Lambda_{-}$, where $\Lambda_{+}$is the eigenspace of $\delta$ with eigenvalue 3 , and $\Lambda_{-}$is the eigenspace of $\delta$ with eigenvalue -1 .

The fibre dimensions are $\operatorname{dim} \Lambda_{+}=2 n^{2}+n$ and $\operatorname{dim} \Lambda_{-}=6 n^{2}-3 n$. Also, $\Lambda_{+}$is the subbundle of $\Lambda^{2} T^{*} M$ of 2 -forms that are of type $(1,1)$ w.r.t. every complex structure $r_{1} I_{1}+r_{2} I_{2}+r_{3} I_{3}$, for $r_{1}, r_{2}, r_{3} \in \mathbb{R}$ and $r_{1}^{2}+r_{2}^{2}+r_{3}^{2}=1$. When $n=1$, the hypercomplex structure induces a conformal structure, and the splitting $\Lambda^{2} T^{*} M=\Lambda_{+} \oplus \Lambda_{-}$is the usual splitting into self-dual and antiself-dual 2-forms.

Following Mamone Capria and Salamon [24, p. 520], we make the following definition.

Definition 4.6.4 Let $M$ be a hypercomplex manifold, let $E$ be a vector bundle over $M$, and let $\nabla_{E}$ be a connection on $E$. Let $F_{E}$ be the curvature of $\nabla_{E}$, so that $F_{E} \in C^{\infty}\left(E \otimes E^{*} \otimes \Lambda^{2} T^{*} M\right)$. We say that $\nabla_{E}$ is a self-dual connection if $F_{E} \in C^{\infty}\left(E \otimes E^{*} \otimes \Lambda_{+}\right)$, that is, if the component of $F_{E}$ in $E \otimes E^{*} \otimes \Lambda_{-}$is zero.

We use the notation $\Lambda_{+}, \Lambda_{-}$and self-dual to stress the analogy with the four-dimensional case, where this notation is already standard. In four dimensions, self-dual connections (also called instantons) are a very important tool in differential topology, and have been much studied. 
The point of the definition is this. If $\nabla_{E}$ is self-dual, then $F_{E}$ is of type $(1,1)$ w.r.t. the complex structure $r_{1} I_{1}+r_{2} I_{2}+r_{3} I_{3}$ by Proposition 4.6.3, and so $\nabla_{E}$ makes $\mathbb{C} \otimes E$ into a holomorphic bundle w.r.t. $r_{1} I_{1}+r_{2} I_{2}+r_{3} I_{3}$. Therefore $\mathbb{C} \otimes E$ has many local holomorphic sections w.r.t. $r_{1} I_{1}+r_{2} I_{2}+r_{3} I_{3}$. But these are just special sections $e$ of $\mathbb{H} \otimes E\left(\right.$ or $\mathbb{I} \otimes E$ ) satisfying $D_{E}(e)=0$.

We deduce that locally, if $F_{E}$ is self-dual, then the equation $D_{E}(e)=0$ admits many solutions. Thus, it is clear that vector bundles with self-dual connections are the appropriate quaternionic analogue of holomorphic vector bundles in complex geometry.

Suppose that $M$ has a notion of q-holomorphic functions of polynomial growth, as in Definition 3.2.6, and that $E$ is equipped with a metric. Sections of $E$ with polynomial growth can then be defined in the obvious way. Let $P_{M, E} \subset Q_{M, E}$ be the filtered AHI-submodule of q-holomorphic sections of $E$ of polynomial growth, and let $P_{M}$ be the filtered $\mathrm{H}$-algebra of q-holomorphic functions on $M$ with polynomial growth. Then $P_{M, E}$ is a filtered module over the H-algebra $P_{M}$.

The ideas of $\$ 3.4$ can also be applied to the problem of reconstructing a self-dual connection from its module $P_{M, E}$. Under suitable conditions, $P_{M, E}$ entirely determines the bundle $E$ and its connection $\nabla_{E}$. This suggests that $\mathrm{H}$-algebra techniques could be used to construct explicit self-dual connections over hypercomplex manifolds of interest.

Now the ADHM construction [1] is an explicit construction of self-dual connections over the hypercomplex manifold $\mathbb{H}$. By Example 4.1.3, each such connection yields a module $P_{M, E}$ over the H-algebra $F^{U}$. The author hopes to study these modules in a later paper, and hence to provide a new treatment and proof of the ADHM construction. The approach generalizes to instantons over other hypercomplex manifolds such as the hyperkähler ALE spaces, for which an ADHM-type construction is given in [21.

\subsection{Directions for future research}

We have developed an extensive and detailed comparison between vector spaces, tensor products and linear maps, and their quaternionic analogues. As a result, many pieces of algebra that use vector spaces, tensor products and linear maps as their building blocks have a quaternionic version. We shall discuss the quaternionic version of algebraic geometry.

Complex algebraic geometry is the study of complex manifolds using algebras of holomorphic functions upon them. In the same way, let 'quaternionic 
algebraic geometry' be the study of hypercomplex manifolds using H-algebras of q-holomorphic functions upon them. I believe that quaternionic algebraic geometry and its generalizations may be interesting enough to develop a small field of algebraic geometry devoted to them.

I have tried to take the first steps in this direction in Chapters 2-4. These methods seem to have no application to compact hypercomplex manifolds, unfortunately, so instead one should study noncompact hypercomplex manifolds satisfying some restriction, such as AC hypercomplex manifolds. The best category of $\mathrm{H}$-algebras to use appears to be FGH-algebras. Here are a number of questions I think are worth further study. Almost all are problems in quaternionic algebraic geometry, and should clarify what I mean by it.

\section{Research problems about hypercomplex manifolds}

- Study the theory of 'coadjoint orbit' hyperkähler manifolds and HPalgebras, begun in $\$ 4.4$, in much greater depth.

- In Example 4.5.6 we saw that hypercomplex manifolds derived from FGH-algebras can have 'invisible singularities' with interesting properties. Study these singularities. Develop a theory of the singularities possible in 'algebraic' hypercomplex manifolds.

- Develop a deformation theory for FGH-algebras. That is, given a fixed FGH-algebra, describe the family of 'nearby' FGH-algebras. One expects to find the usual machinery of versal and universal deformations, infinitesimal deformations and obstructions, cohomology groups. However, the author's calculations suggest that the H-algebra setting makes the theory rather complex and difficult. The application is to deformations of 'algebraic' hypercomplex manifolds.

- Use this deformation theory to construct hyperkähler deformations of singular hyperkähler manifolds. Can you find new explicit examples of complete, nonsingular hyperkähler manifolds of dimension at least 8 ?

- In particular, try and understand the deformations of $\mathbb{H}^{n} / \Gamma$, for $\Gamma$ a finite subgroup of $S p(n)$. In 15 the author used analysis to construct a special class of hyperkähler metrics on crepant resolutions of $\mathbb{C}^{2 n} / \Gamma$. They are called Quasi-ALE metrics, and satisfy complicated asymptotic conditions at infinity. 
It seems likely that these Quasi-ALE metrics are natural solutions to the deformation problem for the FGH-algebra of $\mathbb{H}^{n} / \Gamma$, and thus that one could use hypercomplex algebraic geometry to study them, and even construct them explicitly.

- Study the H-algebras of ALE spaces, mentioned in $\S 4.5$. Use H-algebras to give a second proof of Kronheimer's classification of ALE spaces [17, [18].

- Rewrite the ADHM construction [1] for self-dual connections on $\mathbb{H}$ in $\mathrm{H}$ algebra language, as suggested in \$4.6. Interpreted this way it becomes a beautiful algebraic construction for modules of the H-algebra $F^{U}$ of Example 4.1.3.

- In a similar way, rewrite the ADHM construction on ALE spaces 21] in terms of $\mathrm{H}$-algebras. Consider the possibility of a 'general ADHM construction' for FGH-algebras and algebraic hypercomplex manifolds, that captures the algebraic essence of the constructions of [1] and [21].

\section{Other research problems}

- Understand and classify finite-dimensional HL-algebras, by analogy with Lie algebras.

- Apply quaternionic algebra in other areas of mathematics, to produce quaternionic analogues of existing pieces of mathematics. These need have no connection at all with hypercomplex manifolds. For instance, one can look at a quaternionic version of the Quantum Yang-Baxter Equation, and try and produce quaternionic knot invariants.

- Generalize the quaternionic algebra idea by replacing $\mathbb{H}$ by some noncommutative ring, or more general algebraic object. Can you find any interesting algebraic applications for this? One particularly interesting case is when $\mathbb{H}$ is replaced by a division ring over an algebraic number field, since these rings have a strong analogy with $\mathbb{H}$.

- We have seen that one may associate an H-algebra to a hypercomplex manifold. However, the converse is false, and one cannot in general associate a hypercomplex manifold to an H-algebra. Following $\$ 3.4$, given an $\mathrm{H}$-algebra $P$ generated by finite-dimensional $Q$, one constructs 
a real algebraic variety $M_{P, Q} \subset Q^{\dagger}$. What geometric structure does $P$ induce on $M_{P, Q}$, for general $P$ ? Study these structures.

- In [13] the author constructed nontrivial examples of manifolds with $n$ anticommuting complex structures, for arbitrary $n>1$. Such structures have also been found by Barberis et al. [3], who call them Clifford structures. The programme of this paper may be generalized to Clifford structures. One simply replaces $\mathbb{H}$ by the Clifford algebra $C_{n}$ in the definition of $\mathrm{H}$-algebra, and then to a manifold with a Clifford structure one may associate a ' $C_{n}$-algebra', the analogue of H-algebra. Use this theory to study and find new examples of manifolds with Clifford structures.

\section{References}

[1] M.F. Atiyah, 'Geometry of Yang-Mills fields', Fermi Lectures, Scuola Normale, Pisa, 1979.

[2] M.F. Atiyah and N.J. Hitchin, 'The geometry and dynamics of magnetic monopoles', Princeton U.P., 1988.

[3] M.L. Barberis, I.G. Dotti Miatello and R.J. Miatello, 'On certain locally homogeneous Clifford manifolds', preprint (1995).

[4] R. Bartnik, 'The mass of an asymptotically flat manifold', Comm. Pure Appl. Math. 39 (1986), 661-693.

[5] R.J. Baston, 'Quaternionic complexes', J. Geom. Phys. 8 (1992), 29-52.

[6] V.A. Belinskii, G.W. Gibbons, D.N. Page, C.N. Pope, 'Asymptotically Euclidean Bianchi IX metrics in quantum gravity', Phys. Lett. 76B (1978), 433-435.

[7] K.H. Bhaskara and K. Viswanath, 'Poisson algebras and Poisson manifolds', Pitman Res. Notes in Math. 174, Longman, 1988.

[8] O. Biquard, 'Sur les équations de Nahm et la structure de Poisson des algèbres de Lie semi-simples complexes', to appear in Math. Ann. 
[9] T. Eguchi and A.J. Hanson, 'Asymptotically flat solutions to Euclidean gravity', Phys. Lett. 74B (1978) 249-251.

[10] G.W. Gibbons and S.W. Hawking, 'Gravitational multi-instantons', Phys. Lett. 78B (1978) 430-432.

[11] N.J. Hitchin, 'Polygons and gravitons', Math. Proc. Camb. Phil. Soc. 85 (1979), 465-476.

[12] N.J. Hitchin, A. Karlhede, U. Lindström and M. Roček, 'hyperkähler metrics and supersymmetry', Commun. Math. Phys. 108 (1987) 535589 .

[13] D. Joyce, 'Manifolds with many complex structures', Quart. J. Math. Oxford 46 (1995), 169-184.

[14] D. Joyce, 'Hypercomplex algebraic geometry', Quart. J. Math. Oxford 49 (1998), 129-162.

[15] D. Joyce, 'Quasi-ALE metrics with holonomy $S U(m)$ and $S p(m)$ ', math.AG/9905043. To appear in Annals of Global Analysis and Geometry.

[16] A. Kovalev, 'Nahm's equations and complex adjoint orbits', preprint (1993).

[17] P.B. Kronheimer, 'The construction of ALE spaces as hyperkähler quotients', J. Diff. Geom. 29 (1989) 665-683.

[18] P.B. Kronheimer, 'A Torelli-type theorem for gravitational instantons', J. Diff. Geom. 29 (1989), 685-697.

[19] P.B. Kronheimer, 'A hyper-kählerian structure on coadjoint orbits of a semisimple complex group', J. London Math. Soc. 42 (1990) 193-208.

[20] P.B. Kronheimer, 'Instantons and the geometry of the nilpotent variety', J. Diff. Geom. 32 (1990) 473-490.

[21] P.B. Kronheimer and H. Nakajima, 'Yang-Mills instantons on ALE gravitational instantons', Math. Ann. 288 (1990), 263-307. 
[22] J.M. Lee and T.H. Parker, 'The Yamabe problem', Bull. A.M.S. 17 (1987), 37-91.

[23] P. Li and S.-T. Yau, 'Asymptotically flat complete Kähler manifolds', p. 131-144 in G. Komatsu and Y. Sakane (eds), 'Complex Geometry', Lect. Notes in Pure Appl. Math. 143, Dekker, 1993.

[24] M. Mamone Capria and S.M. Salamon, 'Yang-Mills fields on quaternionic spaces', Nonlinearity 1 (1988) 517-530.

[25] D. Quillen, 'Quaternionic algebra and sheaves on the Riemann sphere', Quart. J. Math. Oxford 49 (1998), 163-198.

[26] S.M. Salamon, 'Riemannian geometry and holonomy groups', Pitman Res. Notes in Math. 201, Longman, 1989.

[27] A. Sudbery, 'Quaternionic analysis', Math. Proc. Camb. Phil. Soc. 85 (1979) 199-225.

[28] D. Widdows, 'Quaternion Algebraic Geometry', Oxford D.Phil. Thesis, 2000. 